ARTICLE

\title{
Plexin-B2 orchestrates collective stem cell dynamics via actomyosin contractility, cytoskeletal tension and adhesion
}

Chrystian Junqueira Alves ${ }^{1}$, Rafael Dariolli2,8, Jonathan Haydak ${ }^{3,8}$, Sangjo Kang ${ }^{1,8}$, Theodore Hannah ${ }^{1,8}$, Robert J. Wiener ${ }^{4}$, Stefanie DeFronzo ${ }^{3}$, Rut Tejero', Gabriele L. Gusella ${ }^{3}$, Aarthi Ramakrishnan', Rodrigo Alves Dias ${ }^{5}$, Alexandre Wojcinski ${ }^{6}$, Santosh Kesari ${ }^{6}$, Li Shen (1) ${ }^{1}$, Eric A. Sobie ${ }^{2}$, José Paulo Rodrigues Furtado de Mendonça ${ }^{5}$, Evren U. Azeloglu (D) 2,3, Hongyan Zou (D) 1,7,9凶 \& Roland H. Friedel (1) 1,7,9凶

During morphogenesis, molecular mechanisms that orchestrate biomechanical dynamics across cells remain unclear. Here, we show a role of guidance receptor Plexin-B2 in organizing actomyosin network and adhesion complexes during multicellular development of human embryonic stem cells and neuroprogenitor cells. Plexin-B2 manipulations affect actomyosin contractility, leading to changes in cell stiffness and cytoskeletal tension, as well as cell-cell and cell-matrix adhesion. We have delineated the functional domains of Plexin-B2, RAP1/2 effectors, and the signaling association with ERK1/2, calcium activation, and YAP mechanosensor, thus providing a mechanistic link between Plexin-B2-mediated cytoskeletal tension and stem cell physiology. Plexin-B2-deficient stem cells exhibit premature lineage commitment, and a balanced level of Plexin-B2 activity is critical for maintaining cytoarchitectural integrity of the developing neuroepithelium, as modeled in cerebral organoids. Our studies thus establish a significant function of Plexin-B2 in orchestrating cytoskeletal tension and cell-cell/cell-matrix adhesion, therefore solidifying the importance of collective cell mechanics in governing stem cell physiology and tissue morphogenesis.

\footnotetext{
${ }^{1}$ Nash Family Department of Neuroscience, Friedman Brain Institute, Icahn School of Medicine at Mount Sinai, New York, NY, USA. ${ }^{2}$ Department of Pharmacological Sciences, Icahn School of Medicine at Mount Sinai, New York, NY, USA. ${ }^{3}$ Division of Nephrology, Department of Medicine, Icahn School of Medicine at Mount Sinai, New York, NY, USA. ${ }^{4}$ Division of Cardiology, Department of Medicine, Icahn School of Medicine at Mount Sinai, New York, NY, USA. ${ }^{5}$ Department of Physics, Federal University of Juiz de Fora, Juiz de Fora, Brazil. ${ }^{6}$ Pacific Neuroscience Institute and John Wayne Cancer Institute at Providence Saint John's Health Center, Santa Monica, CA, USA. ${ }^{7}$ Department of Neurosurgery, Icahn School of Medicine at Mount Sinai, New York, NY, USA. ${ }^{8}$ These authors contributed equally: Rafael Dariolli, Jonathan Haydak, Sangjo Kang, Theodore Hannah. ${ }^{9}$ These authors jointly supervised this work: Hongyan

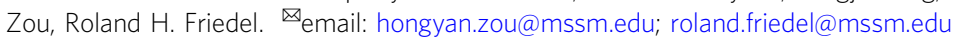


T he multicellular organization relies on biomechanical processes that mediate the generation, transmission, and sensing of mechanical force in cells ${ }^{1-3}$. For instance, during tissue morphogenesis, individual cells constantly rearrange against one another, which requires rapid cytoskeletal adjustment and establishment of a collective actomyosin network and adhesion complexes across cells in order to maintain cell morphology and tissue cohesion ${ }^{4,5}$. Cell-generated forces are transmitted through cell-cell junctions and focal adhesion sites at the interface between the cytoskeleton and extracellular matrix (ECM), which in turn provide important signals to inform cell fate decisions ${ }^{6}$. While a number of mechanotransduction pathways that enable cells to perceive and adapt to physical environment have been mapped ${ }^{1,7}$, the molecular mechanisms orchestrating collective cytoskeletal dynamics are less clear.

One informative example of multicellular dynamics is the selfassembly of cultured human embryonic stem cells (hESCs) into an epithelium-like colony, with stereotypical apical-basolateral polarization, E-cadherin-based adherens junctions, ZO-1containing tight junctions, and integrin-based focal adhesions (FAs) at the interface between cytoskeleton and $\mathrm{ECM}^{8,9}$. During hESC colony formation, a network of cortical actomyosin is formed across cells in the colony interior, while continuous filamentous actin (F-actin) band emerges spanning multiple cells at the colony periphery. This leads to in-plane traction forces that are primarily localized at colony edges, as revealed by traction force microscopy ${ }^{6}$. Another example of complex mechanomorphogenesis is the process of neural tube closure and ventricle formation during neurodevelopment ${ }^{10}$. How hESCs and neuroprogenitor cells (NPCs) orchestrate cytoskeletal tension across cells and organize adhesion complexes at cell-cell and cell-matrix junctions to maintain cytoarchitectural integrity is incompletely understood.

Plexin-B2 is an axon guidance receptor widely expressed in the developing brain, in particular at the ventricular surface, supporting its role in regulating neuroprogenitors ${ }^{11}$. Plexin-B2 plays an essential role in neural tube closure and cerebellar granule cell migration during neurodevelopment ${ }^{12-14}$. Mouse mutant studies also revealed a role of Plexin- $\mathrm{B} 2$ in coordinating the proliferation and migration of neuroblasts in the rostral migratory stream $^{12,13,15,16}$. Plexin-B2 is also expressed in brain tumor cells ${ }^{17}$ and epidermal stem cells ${ }^{18}$. Recent studies have begun to unravel the essential roles of Plexins in mediating cellular interactions in a variety of tissue contexts, including vascular development, immune activation, bone homeostasis, and epithelial morphogenesis and repair ${ }^{18-28}$. However, the underlying mechanisms remain unclear.

Interestingly, phylogenetic analyses unveiled an early evolutionary emergence of Plexins that predates the appearance of nervous systems $\mathrm{s}^{29,30}$. Plexins have a highly conserved domain structure throughout all metazoan clades, particularly the ringshaped extracellular domain and the intracellular Ras-GAP (GTPase activating protein) domain ${ }^{31,32}$. The Ras-GAP domain of Plexins can inactivate Ras or Rap small GTPases ${ }^{33}$, which have a pleiotropic network of effectors that control cytoskeletal dynamics and cell-cell adhesion ${ }^{34,35}$. Hence, we posit that Plexins may have evolved during the transition from unicellular to multicellular organisms to adjust cytoskeletal tension and adhesion forces at cell-cell and cell-matrix junctions ${ }^{29}$.

Here, we show that Plexin-B2 orchestrates multicellular dynamics of hESCs and hNPCs by controlling collective cytoskeletal dynamics and tensional forces. This, in turn, affects the maturation of adhesive complexes at cell-cell and cell-matrix junctions, thereby impacting membrane-association of $\beta$-catenin, focal adhesion, and integrin activation, leading to alteration of cell morphology and tissue geometry, as well as stem cell behaviors.
We further delineate Plexin-B2 signaling domains, the role of RAP1/2 effectors, and the signaling relationship with the mechanosensitive transcription factor YAP (Yes-associated protein $)^{36,37}$, thus providing a mechanistic link between PlexinB2 signaling and stem cell physiology in a multicellular organization.

\section{Results}

Plexin-B2 affects the expansion and geometry of the hESC colony. To better understand the role of Plexin-B2 in a multicellular organization, we generated hESCs with PLXNB2 knockout (KO) by CRISPR/Cas9 gene editing, or Plexin-B2 overexpressing (OE) by lentiviral transduction. Western blot (WB) and immunofluorescence (IF) confirmed loss or gain of Plexin-B2 expression, respectively (Fig. 1a), while DNA sequencing confirmed biallelic CRISPR deletion mutations (Fig. S1a). Introducing a CRISPR-resistant rescue vector restored Plexin-B2 expression in KO cells (Fig. S1b). All CRISPR engineered hESCs displayed normal karyotypes (Fig. S1c).

hESCs under standard culture conditions resemble epiblast, an epithelial layer of pluripotent stem cells (PSCs) in the egg cylinder stage of mammalian embryos ${ }^{38}$. We found that after passage at low density, the initial growth kinetics of hESCs appeared similar among the WT, PLXNB2 KO, or OE conditions, but by day 6 , the $\mathrm{KO}$ colonies became smaller than the WT or OE counterparts (Figs. 1b and S1d). Consistently, 5-ethynyl-2'-deoxyuridine (EdU) pulsing labeled fewer cells in the $\mathrm{S}$ phase in $\mathrm{KO}$ colonies than in WT (Fig. S1e). Reexpressing Plexin-B2 rescued the phenotype, confirming the specificity of the CRISPR-KO approach, while Plexin-B2 OE did not further elevate the high proliferative state of hESCs (Fig. S1e, f). Cell survival was not overtly affected by Plexin-B2 KO, shown by IF for apoptosis marker cleaved caspase 3 (Fig. S1g). Quantitative analysis of the relationship between colony size and Plexin-B2 expression levels showed that PlexinB2 expression remained constant during colony expansion (Fig. S1h). Intriguingly, we noted that the colony geometry was affected in mutant colonies, in particular, the PLXNB2 OE colonies displayed spiky contours along the colony edge, in drastic contrast to the smooth border typically seen in WT colonies (Fig. 1b). Together, these phenotypes indicate that Plexin-B2 critically regulates the colony expansion and geometry of hESCs.

Plexin-B2 affects gene expression concerning cell contraction and ECM interaction. To understand how guidance receptor Plexin-B2 controls colony expansion and geometry on a molecular level, we compared the transcriptomes of PLXNB2 KO and OE hESCs relative to WT by RNA-sequencing (RNA-seq). Bioinformatic analysis revealed 320 differentially expressed genes (DEGs) between PLXNB2 KO and WT, and 2037 DEGs between OE and WT hESCs (Figs. 1c, d, and S2c).

Geneset network analysis showed that the enriched Reactome pathways in both sets of DEGs mainly concerned muscle contraction, elastic fiber formation, ECM organization, and integrin signaling (Fig. 1e). Pathway enrichment analyses further indicated that Plexin-B2 manipulations affected Rap1 signaling, Hippo signaling, and mechanoregulation of YAP/TAZ (Fig. S2a, b). These results suggest that Plexin-B2 may affect colony formation by controlling contractile and adhesive properties, which in turn impacts stem cell physiology. Indeed, bioinformatics analyses revealed that multiple signaling pathways critical for stem cell functions including EGFR, FGFR, MAPK, ERK1/2, Wnt/calcium, and focal adhesion/PI3K/AKT pathways, were all affected by Plexin-B2 manipulations (Figs. 1e and S2a, b). 
a

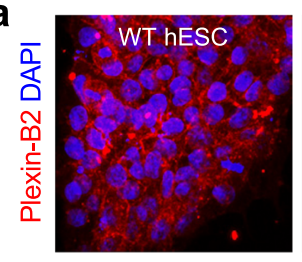

b

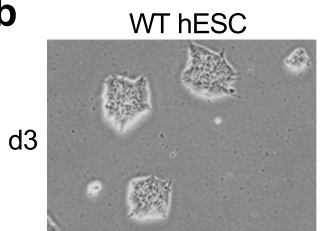

d4

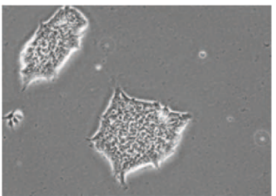

d6
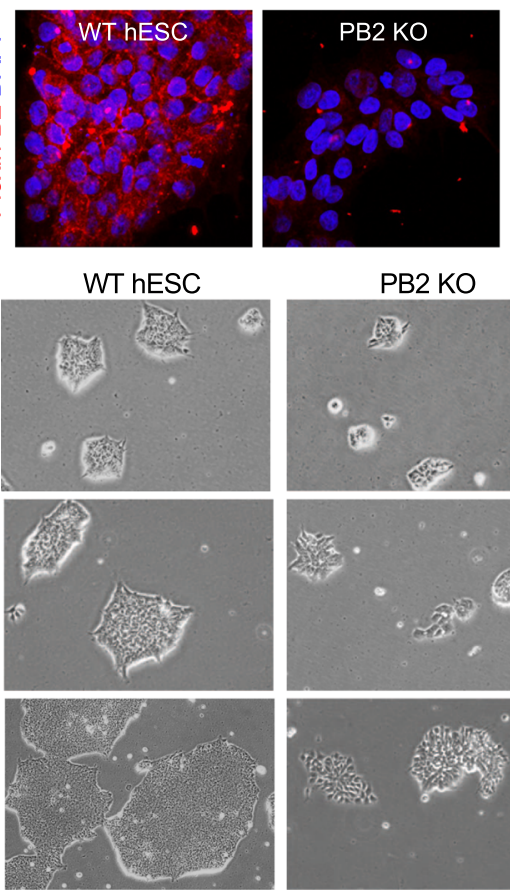

PB2 KO
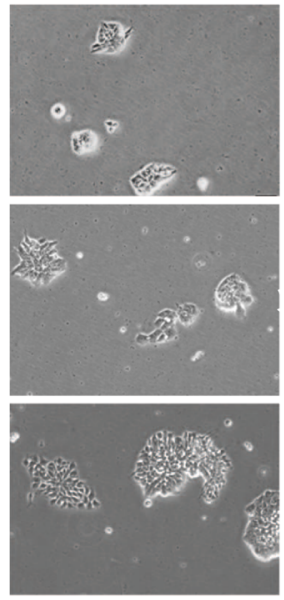

C
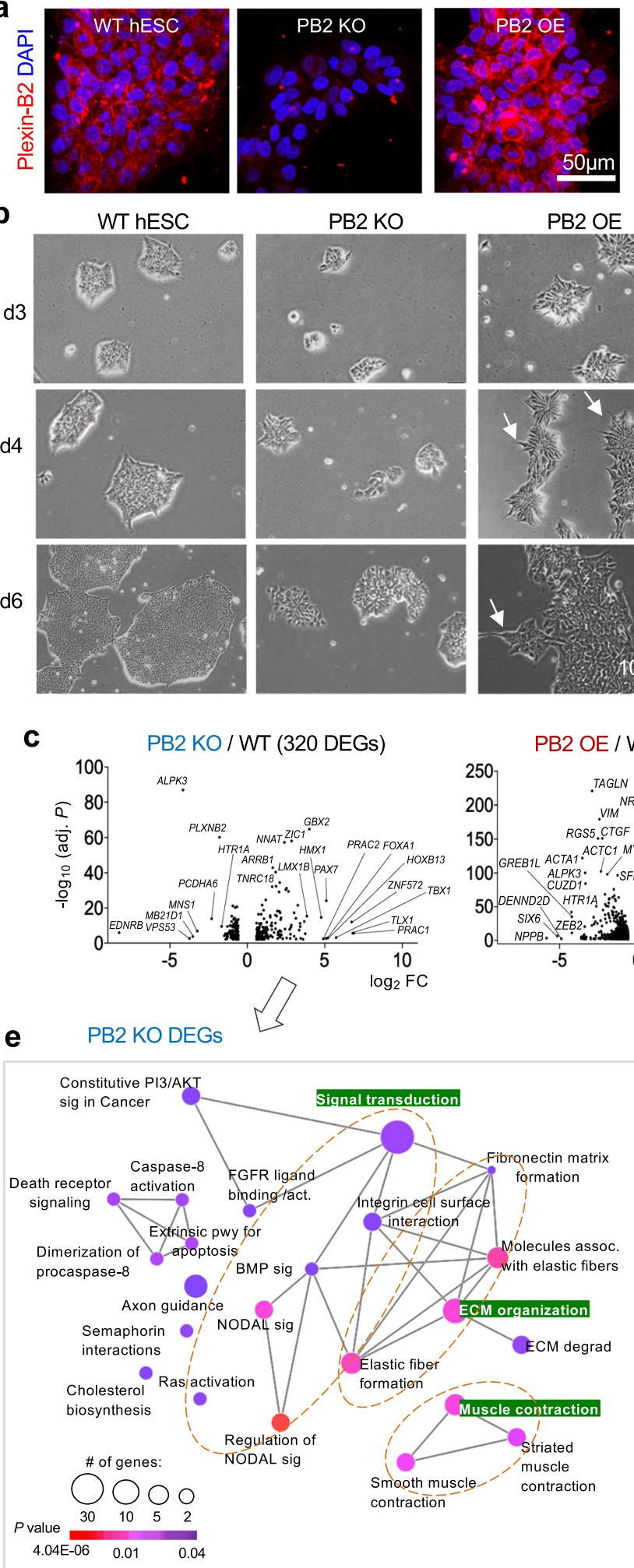
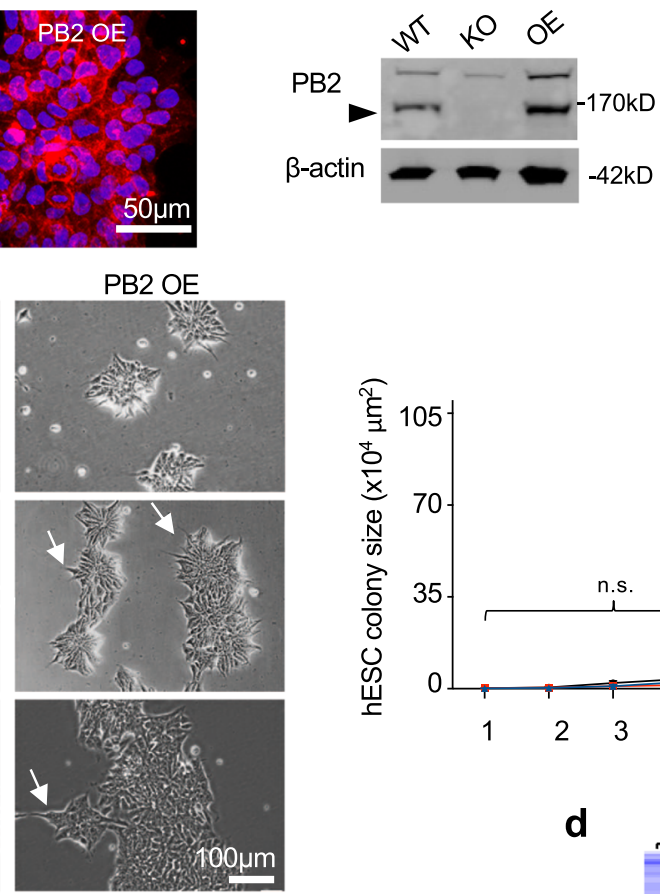

PB2 OE / WT (2037 DEGs)

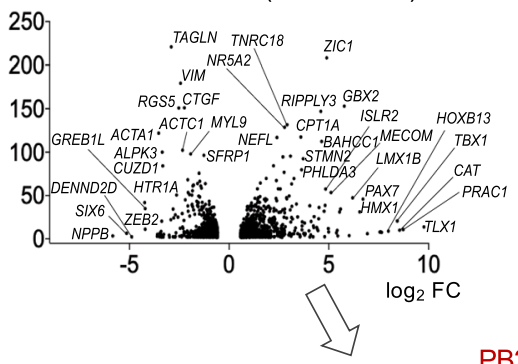

PB2 OE DEGs

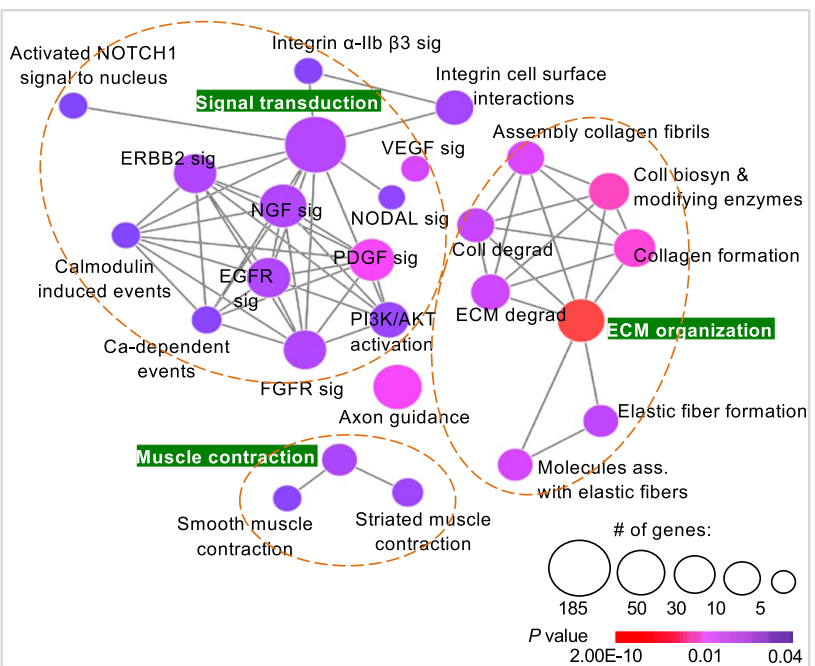

We next intersected the two sets of DEGs (PLXNB2 KO or PLXNB2 OE vs. WT), which revealed 272 common DEGs, mainly concerning axon guidance, contractility, ECM interactions, Ras and Rap signaling, as well as NODAL and BMP signaling (Fig. S2c, d). In regard to nonoverlapping DEGs, Plexin-B2 OE specific DEGs (1765 genes) were enriched for receptor tyrosine kinase (RTK) signaling, including PDGF, EGFR, NGF, VEGF, FGF, and ERBB2 signaling (Fig. S2c). This agrees with the known interactions of Plexins and RTKs ${ }^{39}$. In contrast, Plexin-B2 KOspecific DEGs (48 genes) were enriched for genes related to 
Fig. 1 Plexin-B2 controls growth and geometry of hESC colonies via mechanoregulation. a IF images (left) show expression of Plexin-B2 (PB2) in wildtype hESC colony (clone WT\#1), which was absent in PLXNB2 KO (clone KO\#1) and enhanced in overexpression (OE) hESCs, respectively. Right, Western blot of hESCs probed for Plexin-B2. Arrowhead points to mature Plexin-B2 band at $170 \mathrm{kD}$. $\beta$-actin as a loading control. Quantification, one-way ANOVA followed by Dunnett's multiple comparisons test. $n=12$ samples per group. arb.units arbitrary units. ${ }^{\star} P=0.0231,{ }^{\star \star} P=0.0034$. Data represent mean \pm SEM. b Phase-contrast images of the indicated hESC colonies show similar initial growth kinetics after passage, but by day $6, P L X N B 2$ KO colonies appeared smaller (clone \#1 for each WT and KO shown). Arrows point to spiky contours of OE colonies. Quantification of colony size is shown on right. Two-way ANOVA followed by Tukey's post hoc test. For WT colonies, $n=14,14,9,10,18,7$ for day 1-6, respectively; for $P L X N B 2 K O, n=19,14,12,14,29$, 25; and for PLXNB2 OE, $n=25,15,11,17,26,23$ colonies, from three independent cultures. ${ }^{\star} P=0.0261$; n.s. not significant. Data represent mean \pm SEM. c Volcano plots show differentially expressed genes (DEGs) of PLXNB2 KO or OE hESCs relative to WT (cut-off thresholds: fold change $>2$ and $P<0.01$ ). Top DEGs are labeled. The $P$ values were computed by the DESeq2 package using the Wald test. $\mathbf{d}$ Heatmap shows unsupervised hierarchical clustering of the DEGs in different genotype conditions (fold change $\geq 2$ between genotypes for the DEGs). e Geneset network view of enriched Reactome terms for DEGs in PLXNB2 KO or OE hESCs relative to WT. Note that gene pathways related to cell biomechanics were highly represented, as were many growth factor signaling pathways, such as EGF and FGF, that are critical for stem cell proliferation and differentiation. Highlighted in green are three major enriched pathways for the DEGs. The $P$ values of node connectivity were computed by the Mann-Whitney-Wilcoxon test.

neuronal functions, e.g., neurotransmitter clearance, metabolism of serotonin, or dopamine degradation, suggesting neuronal lineage commitment of $\mathrm{KO}$ cells.

Interestingly, a majority of the common DEGs exhibited transcriptional changes in the same direction in both $\mathrm{KO}$ and $\mathrm{OE}$ hESCs relative to WT (Fig. S2d), suggesting that the gene expression changes likely reflect secondary adaption in response to altered cell biomechanics rather than direct transcriptional regulation by Plexin-B2 signaling. A small number of DEGs displayed opposite directionality of the transcriptional changes in $\mathrm{KO}$ and $\mathrm{OE}$ cells (Fig. S2d), including genes regulating stem cell function and matrix interactions, e.g., TNC (Tenascin C), an ECM gene involved in neuronal differentiation ${ }^{40}$, and CER1 (Cerberus1), a BMP antagonist essential for inhibiting nonneural differentiation of hESCs, as well as CCDC141 (coiled-coil domain containing 141), which inhibits cellular adhesion to fibronectin (FN), and TACR3 (Tachykinin receptor 3), a G protein-coupled receptor that regulates neuronal excitation and smooth muscle contraction.

Plexin-B2 orchestrates actomyosin network and cytoskeletal tension during hESC colony formation. The transcriptomics results indicated cell mechanics as a potential mechanism underlying Plexin-B2's function in hESCs. We, therefore, examined the F-actin networks in hESC colonies by phalloidin staining (Fig. 2a). In WT colonies, we observed a uniform cobblestone-like cortical F-actin network in the colony interior, and a prominent F-actin band spanning multiple cells along colony periphery, consistent with higher in-plane traction forces at colony edge 6 . In comparison, the organization of F-actin networks was severely disrupted by Plexin-B2 $\mathrm{KO}$ or $\mathrm{OE}$, albeit in different ways (Fig. 2a). In the $\mathrm{KO}$ colonies, we found that mutant cells tended to aggregate into small cell clusters, with F-actin accumulated at the center, while the peripheral F-actin band was absent, signifying dysregulation of cytoskeletal tension during colony formation. In the $\mathrm{OE}$ colonies, cells at the colony periphery were highly disorganized, forming spiky contours, and F-actin distribution was in disarray throughout the colonies, with many cells showing strengthened cortical F-actin and stretched cell borders. Indeed, quantification clearly demonstrated a more homogenous cellular arrangement in WT colonies than in mutants (Fig. 2b). As tissue tension is primarily generated by actomyosin contraction and initiated by phosphorylated myosin light chain 2 (pMLC2) $)^{9,41}$, we examined pMLC2 expression, which displayed marked disarray in mutant colonies, mirroring the F-actin patterns (Fig. 2a). Thus, Plexin-B2 has a critical role in organizing the actomyosin network and tensional forces during hESC colony formation.
To examine cell morphology and F-actin networks with a higher spatiotemporal resolution during colony formation, we performed time-lapse live-cell imaging, using SPY-actin to label F-actin ${ }^{42}$. We found that PLXNB2 OE cells assumed a striking angular morphology with stretched cortical F-actin, taut cell borders, and larger cell area as compared to WT hESCs, whereas PLXNB2 KO cells exhibited less cortical F-actin, a more rounded shape, but comparable size relative to WT (Figs. 2c and S3a). As the cytoskeleton configuration may affect the size and arrangement of organelles, we compared the shape and size of nuclei in hESC colonies and found that PLXNB2 KO hESCs had smaller nuclei than WT cells, whereas PLXNB2 OE cells had more stretched and larger nuclei than WT cells (Fig. S3b).

Remarkably, live videography also revealed that hESCs were highly motile, with constant collisions between small cell clusters before they coalesced into larger colonies, and during this process, cells collectively organized their cortical F-actin networks into a cobblestone-like pattern in the colony interior and a prominent circumferential F-actin band spanning multiple cells at colony periphery (Supplementary Movie 1); this orchestration was severely disrupted by Plexin-B2 perturbations. Specifically, upon collision, PLXNB2 OE cells maintained an angular morphology and appeared sluggish in reorganizing F-actin networks, leading to stretched junctional borders and a spiky contour in nascent colonies. In contrast, PLXNB2 KO cells tended to aggregate into smaller clusters with a central accumulation of F-actin; upon collision, cells in the colony center remained disorganized, while the peripheral F-actin band failed to form.

We further analyzed cell motility during colony expansion by live-cell imaging, which showed that cells at the colony periphery migrated faster on average than those in the colony interior (Fig. S3c). Interestingly, the movement of cells at colony edge in Plexin-B2 OE conditions was highest among the three genotypes.

To gain temporal control, we introduced two independent doxycycline (Dox)-inducible shRNAs against PLXNB2 into hESCs (Fig. S3d). As in KO, Dox-induced Plexin-B2 knockdown (KD) resulted in disorganization of the actomyosin network and dissipation of the peripheral F-actin band (Fig. S3e, f). The altered colony geometry and cellular misalignment at the edges of $\mathrm{KD}$ colonies could be reversed by $24 \mathrm{~h}$ of Dox washout, indicating rapid cytoskeletal reorganization under the control of Plexin-B2 (Fig. S3g).

To directly gauge mechanical properties of hESC colonies in dependence of Plexin-B2, we conducted microindentation testing (elastography) using atomic force microscopy (AFM) ${ }^{43}$. Results showed that PLXNB2 KO cells had lower stiffness (mean $2.0 \mathrm{kPa}$ ) than WT $(2.2 \mathrm{kPa})$, but PLXNB2 OE cells had higher stiffness (3.1 kPa) (Fig. 2d). We also performed 3D contact imaging by AFM to scan cell surface topologies, which revealed rougher 
a
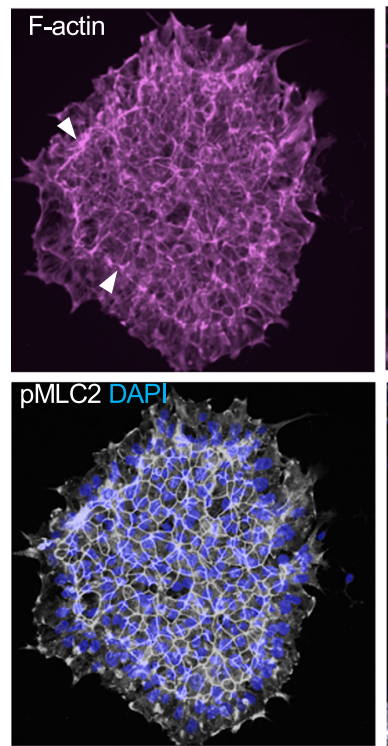

c

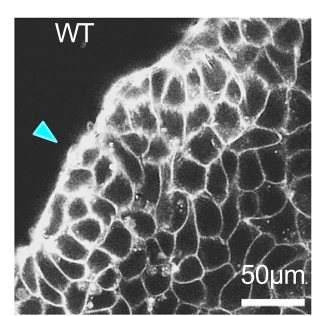

PB2 KO
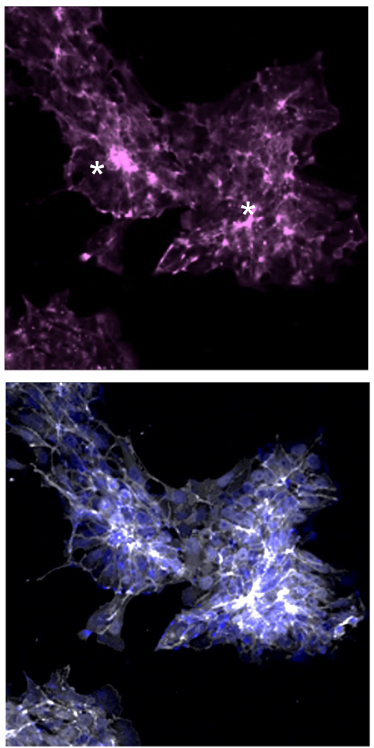

Live: SPY-actin
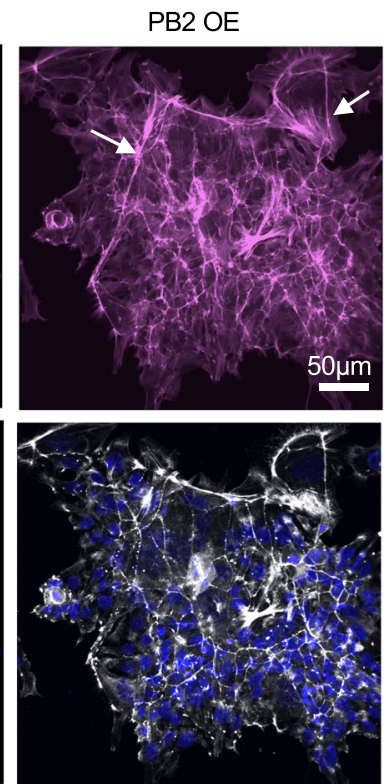

b

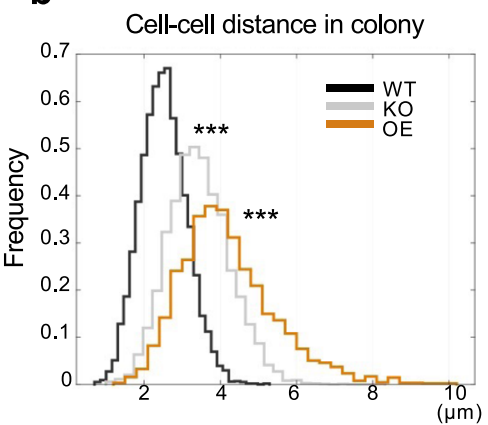

Colony circularity Colony solidity

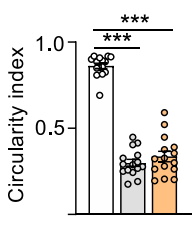

WT KO OE
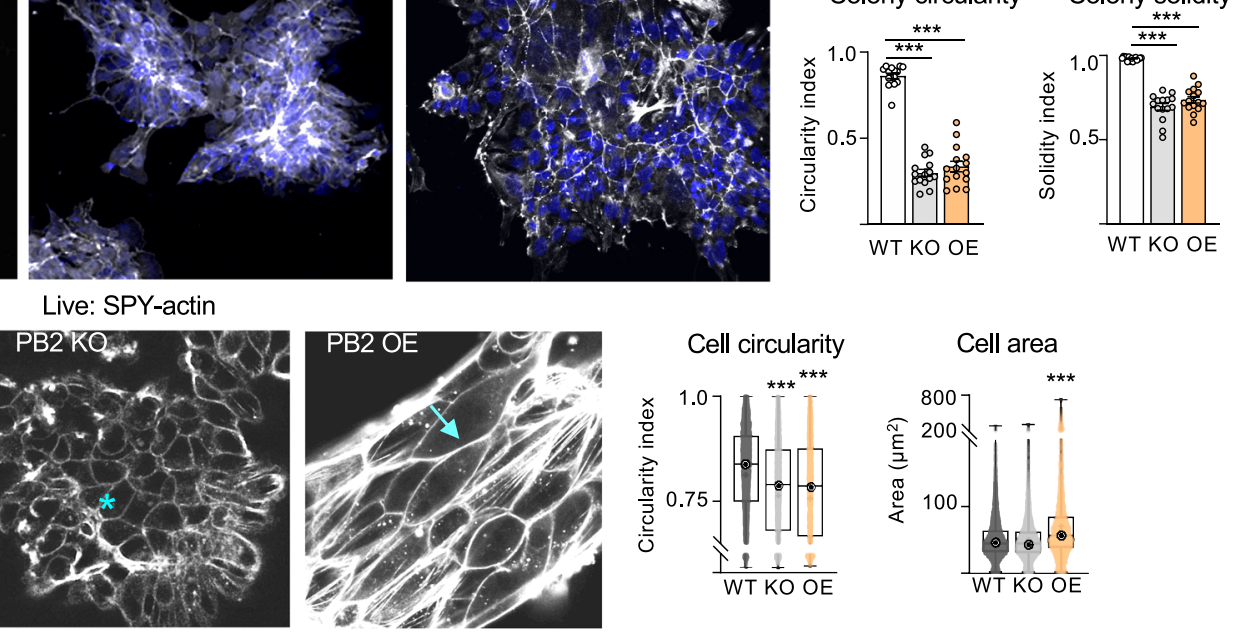

d

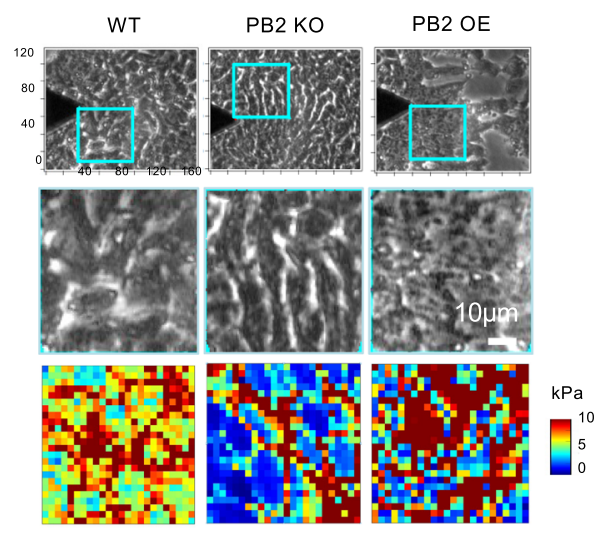

e

WT

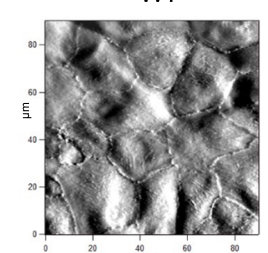

PB2 KO

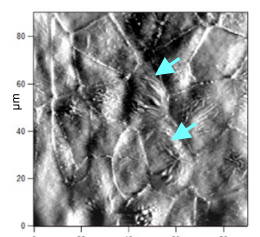

PB2 OE

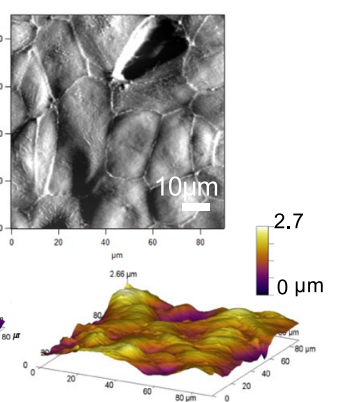

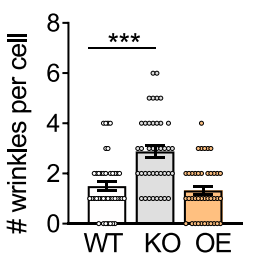
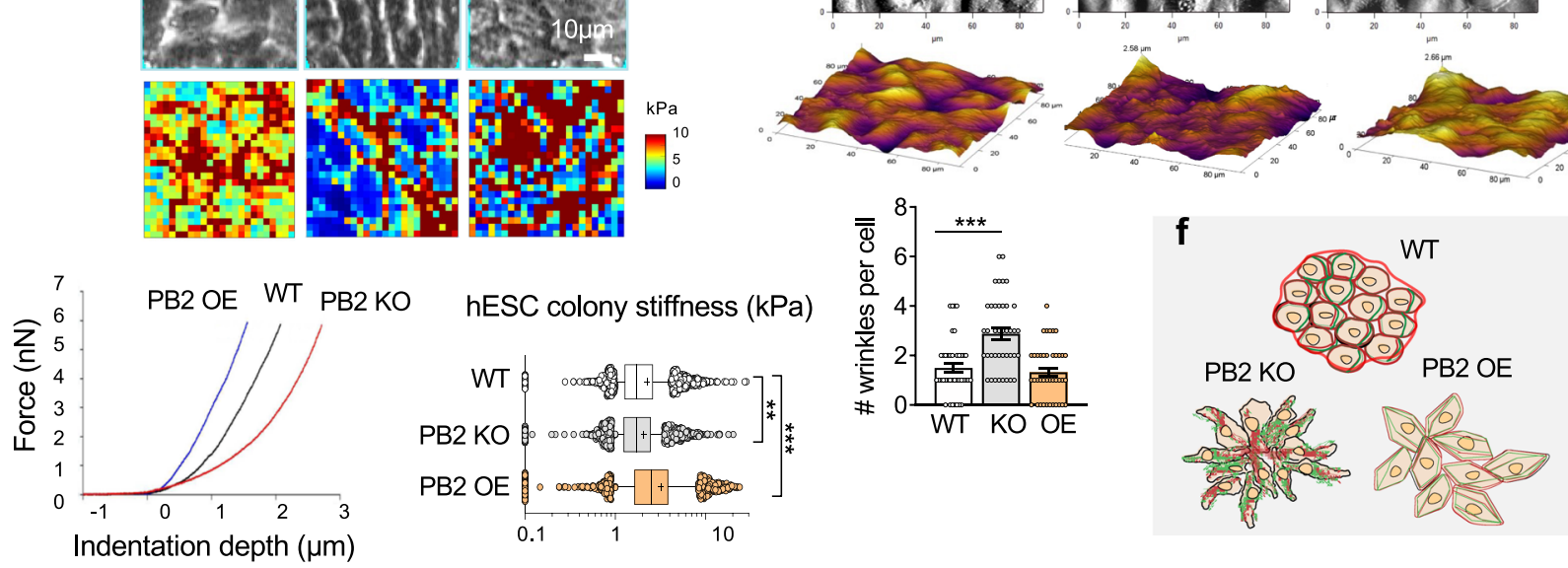

and neuroprogenitor migration ${ }^{12}$, we next investigated whether Plexin-B2 controls biomechanical properties of hNPCs. To this end, we derived hNPCs from WT, PLXNB2 KO, or OE hESCs and analyzed actomyosin patterns. Similar to the results from hESCs, cortical actomyosin accumulation was reduced in PLXNB2 KO hNPCs relative to WT, but increased in OE cells (Fig. 3a, b). Morphometric quantifications confirmed alterations of cell shape as a result of Plexin-B2 manipulations (Fig. 3b). Interestingly, the nuclei of both PLXNB2 KO and OE hNPCs 
Fig. 2 Plexin-B2 orchestrates mechanotension and actomyosin network in hESC colonies. a IF images of hESC colonies stained for F-actin (Alexa 568 phalloidin) and pMLC2 show differences in cell organization, colony geometry, and actomyosin network. Arrowheads point to the peripheral actomyosin band in the WT colony, which was disrupted in PLXNB2 KO or OE mutants. Asterisks denote abnormal cell clusters in the KO colony. Arrows point to stretched contours of the OE colony, often associated with F-actin stress fibers. DAPI for nuclear staining. $\mathbf{b}$ Top, histograms show the distributions of distances between neighboring cells within hESC colonies (distances between nucleus centroids). Note the wider distribution of cellular distances in KO or OE colonies than in WT, reflective of disorganization. Bottom, quantification of colony geometry (circularity index and solidity). For cell distance histogram, Kruskal-Wallis test followed by Dunn's multiple comparisons test, $n=7646$ nuclei for WT, 4478 for PLXNB2 KO, and 2964 for $P L X N B 2$ OE. ${ }^{\star \star \star} P<0.0001$. For colony geometries, one-way ANOVA followed by Dunnett's multiple comparisons test, $n=15$ colonies per group. ${ }^{\star \star \star} P<0.0001$. Data represent mean \pm SEM. c Live-cell imaging of hESC colonies show Plexin-B2-dependent differences in cell morphology, cell organization, and cortical F-actin visualized with SPY555-Actin probe. Arrowhead points to the peripheral F-actin band in WT colony, an asterisk denotes abnormal cell cluster in PLXNB2 $\mathrm{KO}$ colony (clone KO\#2), and arrow points to tensed cortical F-actin and stretched junction borders in PLXNB2 OE colony. High content quantifications of cell shape (circularity index) and cell area are shown. Box plots show 25-75\% quantiles, minimal and maximal values (whiskers), and median (bullseye). Kruskal-Wallis test followed by Dunn's multiple comparisons test. For circularity: WT, $n=7878$ cells; PLXNB2 KO, $n=5471$, and PLXNB2 OE, $n=3904$. For area: WT, $n=9725$ cells; PLXNB2 KO, $n=5615$, and PLXNB2 OE $n=4051$. ${ }^{\star \star \star} P<0.0001$. Data represent mean \pm SEM. $\mathbf{d}$ Top panel, phase-contrast images show areas in hESC colonies (cyan boxes) measured by AFM elastography. Middle panels, side-by-side magnified phase-contrast images, and corresponding AFM stiffness heatmaps. Bottom, representative AFM indentation curves from elastography measurements (left) and box plots of hESC colony stiffness (right). Box plots show median, 25-75\% quantiles and top and bottom 5\% data points; mean values indicated by a cross sign. Kruskal-Wallis test followed by Dunn's multiple comparisons test. Data collected from $n=7$ samples for WT, $n=6$ for PLXNB2 KO, and $n=7$ for PLXNB2 OE. ${ }^{\star \star} P=0.0032,{ }^{\star \star \star} P<0.0001$. e Top, AFM topography images show increased surface wrinkles in PLXNB2 KO cells (arrows), but more stretched surfaces of PLXNB2 OE cells compared to WT hESCs. Bottom, quantification of the number of surface wrinkles per cell. One-way ANOVA followed by Dunnett's multiple comparisons test. $n=40$ cells analyzed per group. ${ }^{\star \star \star} P<0.0001$. Data represent mean \pm SEM. f Schematic depiction of cell morphology, cell organization, actomyosin network, and colony geometry as regulated by Plexin-B2 during hESC self-assembly. Red lines indicate F-actin and green lines myosin.

were on average smaller than those of WT counterparts (Fig. 3b), which may be associated with an aberrant differentiation state.

We next performed live-cell imaging of hNPCs labeled with mScarlet-Lifeact, a fluorescent F-actin probe ${ }^{44}$. We detected reduced cortical F-actin in PLXNB2 KO hNPCs, but a marked elevation in $\mathrm{OE}$ cells that contained actin stress-fibers and angular morphology (Fig. 3c). AFM elastography demonstrated that PLXNB2 OE NPCs exhibited higher cell stiffness (mean $6.3 \mathrm{kPa}$ ) than WT hNPCs $(5.9 \mathrm{kPa})$; conversely, cell stiffness was lower for KO hNPCs (4.0 kPa) (Fig. 3d).

To measure tension exerted on FAs in hNPCs, we introduced a FRET tension sensor based on vinculin, an intracellular linker of adhesion sites with cytoskeleton ${ }^{45}$ (Fig. 3e). Analysis of the FRET signals revealed that the forces exerted on FAs were indeed lower in PLXNB2 KO than in WT hNPCs, but higher in OE hNPCs (Fig. 3e).

Plexin-B2 affects the organization of adhesion complexes in epithelial colonies and 3D hESC aggregates. So far, we showed that Plexin-B2 controls cell stiffness and actomyosin organization in the multicellular organization. As contractile forces are transmitted to cell-cell and cell-matrix junctions to maintain tissue cohesion $^{3}$, we next studied the impact of Plexin-B2 on the organization of adhesion complexes in hESC colonies. We observed that while E-cadherin and ZO-1 displayed a uniform cobblestone-like network in WT colonies, both were severely disrupted in PLXNB2 mutant colonies (Figs. 4a and S4a), concordant with the cellular disarray and disorganized actomyosin networks.

In hESCs, $\beta$-catenin forms a complex with E-cadherin ${ }^{46}$, which connects to actin cytoskeleton to mediate cell-cell adhesion and pluripotency $^{8}$. Indeed, in WT hESCs, $\beta$-catenin displayed a similar cobblestone-like pattern as E-cadherin (Fig. 4b). However, Plexin-B2-deficiency resulted in reduced membrane association of $\beta$-catenin and redistribution towards the center of cell clusters, a phenotype partially rescued by Plexin-B2 re-expression; in contrast, Plexin-B2 OE led to an increased nuclear fraction of $\beta$ catenin (Fig. 4b). These phenotypes are likely a consequence of the disorganized membrane clustering of E-cadherin in hESC colonies. WB confirmed a shift in subcellular localization of $\beta$ - catenin (Fig. 4c), while the total protein levels remained similar in all three groups (Fig. 4d). The subcellular fractionation approach was verified by probing for markers of nuclear, cytoplasmic, and membrane fractions, respectively (Fig. S4b).

We next examined the impact of Plexin-B2 on the selfassembly of hESCs in a $3 \mathrm{D}$ context. When cultured in lowattachment dishes, WT hESCs spontaneously aggregated into compact spheroids of $\sim 200-500 \mu \mathrm{m}$ diameter by $48 \mathrm{~h}$; strikingly, PLXNB2 KO cells formed larger but irregularly shaped aggregates, measuring up to $1.5 \mathrm{~mm}$ in length, a phenotype rescued by Plexin-B2 reexpression (Fig. 4e). Conversely, PLXNB2 OE resulted in smaller but more compact aggregates $(\sim 18-60 \mu \mathrm{m}$ in diameter), reflective of a heightened contractile state. As a gauge of tissue cohesion or adhesive forces between cells, we tested the ability of hESC aggregates to resist external shear stress applied either through gentle pipetting or by centrifugation ${ }^{47}$. Results showed that whereas WT aggregates maintained the compact spheroid shape under shear stress, the large PLXNB2 KO aggregates appeared easily broken up into smaller pieces with uneven shapes, consistent with reduced tissue cohesion (Fig. 4f).

Concordantly, IF staining of cross sections of the WT spheroids revealed a high concentration of organized F-actin networks in the center, surrounded by a rim of pMLC2 at the periphery, whereas both F-actin and pMLC2 appeared more compact in PLXNB2 OE spheroids, but looser and in disarray in $\mathrm{KO}$ aggregates (Fig. 4g). Actomyosin contraction can affect FN assembly in multicellular organization ${ }^{48,49}$. Indeed, as another sign of disorganized actomyosin network, the gradient of FN fibrils extending from core to periphery seen in WT spheroids appeared more compact in PLXNB2 OE spheroids, but in marked disarray in $\mathrm{KO}$ aggregates (Fig. S4c).

As an alternative approach to gauge cell-cell cohesion, we also examined aggregation of hESCs and hNPCs in a hanging drop assay $^{50}$. We detected reduced or enhanced compaction of hanging drop aggregates of PLXNB2 $\mathrm{KO}$ or OE cells, respectively, relative to WT counterparts (Fig. S4d, e).

Plexin-B2 affects focal adhesion maturation in hESC colony and hNPCs. We next examined the impact of Plexin-B2mediated actomyosin tension on the interface of cytoskeleton 
a
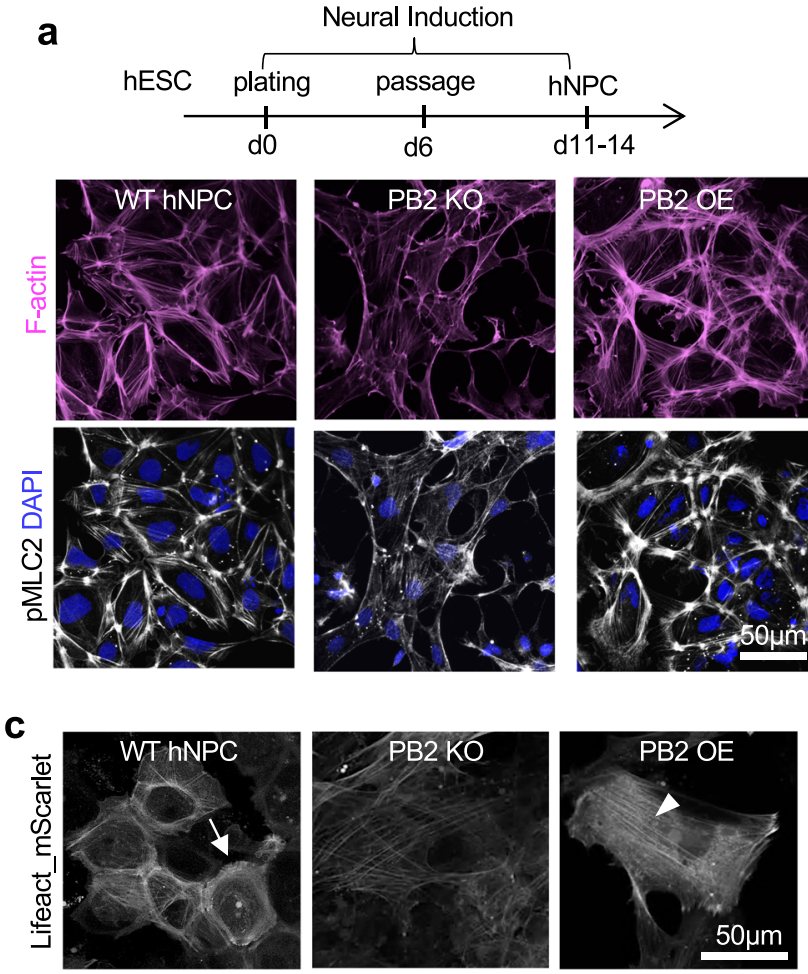

b
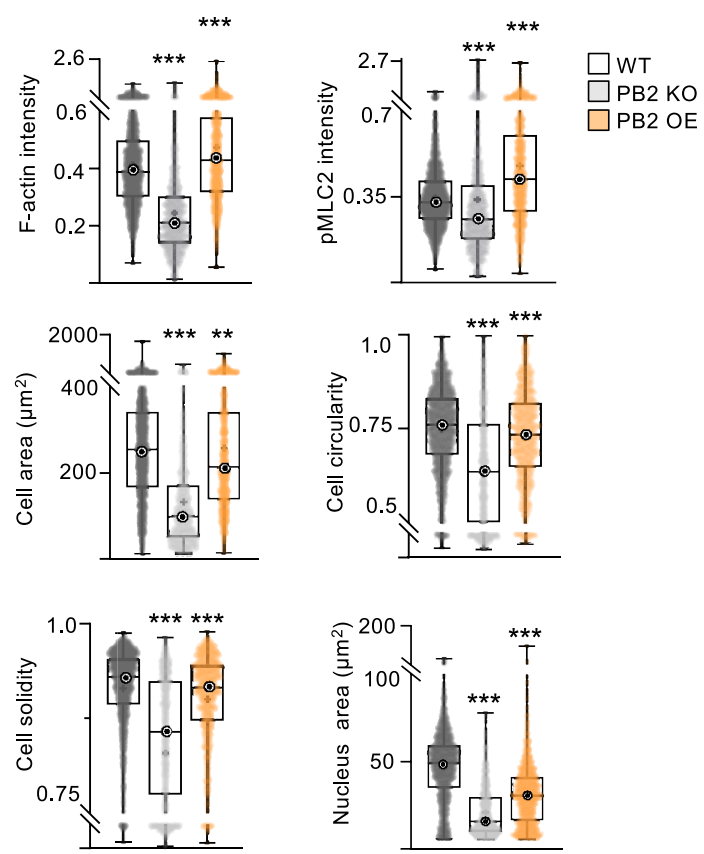

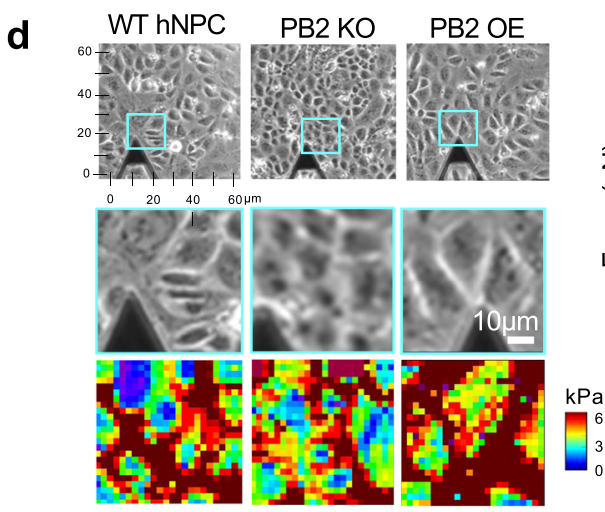

e
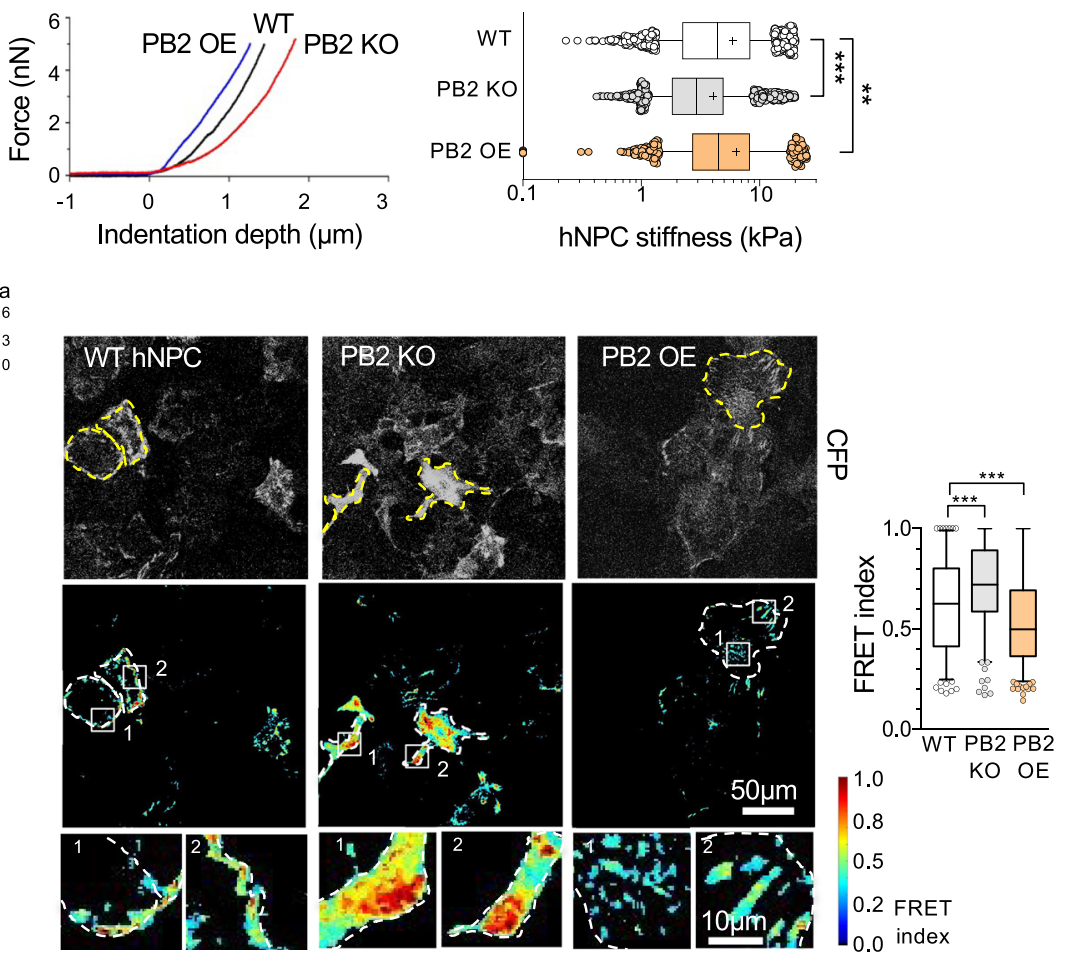

and matrix. We performed total internal reflection fluorescence (TIRF) microscopy for morphometric analysis of FAs visualized by paxillin IF (Fig. 5a, b). In both hESCs and hNPCs, we observed increased numbers of FAs with PLXNB2 OE. In addition, both the size and the shape of FAs were also altered, with generally larger FAs with PLXNB2 OE, and less circular ones with KO, although there were variabilities between hESCs and hNPCs (Fig. 5a, b).

Next, we investigated the levels of phosphorylated focal adhesion kinase $(\mathrm{pFAK})$ as an indicator of stable FAs. Confocal 
Fig. 3 Plexin-B2 regulates actomyosin contractility and cell stiffness in hNPCs. a Top, timeline of neural induction to generate hESC-derived hNPCs. Bottom, confocal IF images of hNPCs show differences in F-actin (phalloidin) and pMLC2 in PLXNB2 KO and OE compared to WT cells. b Box plots show high content quantifications of F-actin and pMLC2 intensity, as well as geometries (cell spreading area, circularity, and solidity) and nuclei area of WT, PLXNB2 KO, and OE hNPCs. Box plots show 25-75\% quantiles, minimal and maximal values (whiskers), median (bullseye), and mean (cross sign). For intensities and cell geometries: WT, $n=2123$ cells; PLXNB2 KO, $n=930$, and PLXNB2 OE, $n=1172$. For nuclei area: WT, $n=2265$ nuclei; PLXNB2 KO, $n=1275$, and PLXNB2 OE, $n=1720$. Data collected from 30 random fields per group. Kruskal-Wallis test followed by Dunn's multiple comparisons test. ${ }^{\star \star} P=0.0037$ and ${ }^{\star \star \star} P<0.0001$. $\mathbf{c}$ Live-cell imaging of hNPCs shows different F-actin distribution patterns in mutant hNPCs as revealed by Lifeact_mScarlet. Arrow points to cortical F-actin in WT hNPCs, which was reduced in PLXNB2 KO cells. Arrowhead points to stress fibers in PLXNB2 OE hNPCs. d Top, phase-contrast images depicting areas of hNPC cultures (cyan boxes) that were measured by AFM elastography. Bottom, side-by-side magnified phase-contrast images and corresponding AFM stiffness heatmaps. Right, representative AFM indentation curves from elastography measurements of WT, PLXNB2 KO, and OE hNPCs (left), and right, box plots quantification of cell stiffness (median, 25-75\% quantiles, median, and top and bottom $5 \%$ data points, with mean values indicated by cross sign). Kruskal-Wallis test followed by Dunn's multiple comparisons test. Data collected from $n=3$ samples per group. ${ }^{\star \star} P=0.0013$, ${ }^{\star \star \star} P<0.0001$. e Left, diagram of vinculin-based FRET tension sensor (CFP and YFP proteins connected by elastic linker) to gauge tensile forces at focal adhesions (FAs). Stretching of the linker reduces FRET signals. Middle, representative images of normalized FRET signals in hNPCs. Note that transient transfection leads to variable expression of FRET construct in cells, as visualized by CFP baseline fluorescence. Right, quantification of FRET index shown as box plots (median, 25-75\% quantiles, and top or bottom 5\% data points). Kruskal-Wallis test followed by Dunn's multiple comparisons test. Data collected from 175 FAs for WT, 191 FAs for PLXNB2 KO, and 268 FAs for PLXNB2 OE. ${ }^{\star * \star P}=0.0001$ (WT vs PB2 KO) and ${ }^{\star \star \star} P=0.0008$ (WT vs PB2 OE).

microscopy revealed diminished levels of pFAK in Plexin-B2deficient hESC colonies, but enhanced accumulation in Plexin-B2 $\mathrm{OE}$, both in disarray in contrast to the cobblestone-like pattern seen in WT (Fig. S5a). In hNPCs, Plexin-B2 OE also led to increased pFAK levels (Fig. S5b). Together, these data support the notion that FA maturation is affected by different tensional forces as mediated by Plexin-B2.

In Drosophila sensory neurons, Plexin-B forms a complex with integrin $\beta$ and stabilizes it on the cell surface to regulate selfavoidance and dendritic tiling ${ }^{51}$. Our microscopy analysis of hESC colonies revealed analogous results, with active integrin $\beta 1$ immunosignals attenuated in PLXNB2 KO, but enhanced in OE colonies (Fig. S5c). Of note, WB showed that only the intracellular localization, but not the total protein level of integrin $\beta 1$ was affected by PLXNB2 KO (Fig. S5d). A similar loss of active integrin $\beta 1$ was observed in PLXNB2 KO hNPCs (Fig. S5e). Hence, Plexin-B2 controls actomyosin tension and maturation of junctional adhesions in multicellular organization (Fig. S5f).

Plexin-B2 primarily controls contractility in hESC colony with consequent changes in adhesion. Our next question pertains to whether Plexin-B2 primarily controls actomyosin contractility or adhesive strength, two interconnected processes in a multicellular organization. To address this question, we performed mathematical simulations of cell mechanics using the molecular dynamics approach $^{52,53}$. We first developed a membrane model that reproduces the main elements of cellular mechanical components based on the coarse-grained bead-spring model, wherein cell membrane, nuclear membrane, and actin filaments were modeled as beads connected by springs; and a virtual AFM tip was represented as a sphere anchored by two springs (Fig. 5c). We simulated how cell stiffness might behave in response to either: (i) modulation of actomyosin contractile forces, as simulated by altering the attraction energy between actin filament heads and cell membrane (energy ع03) or (ii) varying cell adhesion, as simulated by altering the interaction energy between neighboring cell membranes $(\varepsilon 00)$. We tested two scenarios: variable $\varepsilon 03$ with fixed $\varepsilon 00$, or variable $\varepsilon 00$ with fixed $\varepsilon 03$ (Fig. $5 c$ and Supplementary Movies 2, 3). The simulation results from the first scenario-i.e., modulating actomyosin contractile forces-aligned better with our experimental AFM data. Hence, mathematical simulations support the model that Plexin-B2 primarily controls actomyosin contractility, with secondary effects on cell adhesiveness during the multicellular organization.
To corroborate the simulation results, we assayed the contractile properties of hESCs in the single-cell state under adherent vs. non-adherent conditions, thus decoupling actomyosin contraction from cell-cell or cell-matrix adhesion (Fig. 5d). Live-cell imaging revealed that under adherent conditions, individual cells with PLXNB2 OE assumed a highly branched morphology, whereas KO cells frequently extended cellular protrusions, suggestive of reduced cell-matrix anchorage. We then released cells from matrix anchorage by incubation with an anti-integrin $\beta 1$ blocking antibody. This resulted in rounding-up of cells, and notably, PLXNB2 OE cells displayed membrane blebbing, signifying cytoskeletal hypercontractility (Fig. 5d). This agrees with the model that a main function of Plexin-B2 is to promote actomyosin contractility.

To further test this model, we applied WT or PLXNB2 mutant hESCs pharmacological inhibitors for myosin II (blebbistatin, $10 \mu \mathrm{M})$ or Rho-associated coiled-coil-containing kinase (ROCK) $(\mathrm{Y}-27632,20 \mu \mathrm{M})^{54}$ (Fig. S6a, b). Inhibition of actomyosin contractility by these inhibitors indeed abolished the hypercontractile state of Plexin-B2 OE cells, leading to junction changes resembling the $\mathrm{KO}$ phenotypes in all three genotype conditions (Fig. S6b). Exposure to latrunculin $(5 \mu \mathrm{M})$, which inhibits F-actin assembly, resulted in rounding-up of cells and cell dissociation in all three groups (Fig. S6b). These data support the interconnection of actomyosin contractility and cell adhesion with a primary function of Plexin-B2 in promoting the former. Interestingly, treatment with Y16 $(25 \mu \mathrm{M})$, a specific inhibitor of the Rho-GEFs, which can function as downstream effectors of Plexin-Bs ${ }^{55}$, caused no significant morphological changes in hESC colonies or in $3 \mathrm{D}$ spheroids in all three groups (Fig. S6c, d).

We performed additional mathematical simulations of molecular dynamics to probe the impact of varying actomyosin contractility on cell cohesion in multicellular settings. This was interrogated by calculating the force needed to pull cells from the colony edge or from the colony center. Simulation results indicated that increased cell contractility can strengthen cell-cell cohesiveness (Fig. S6e). Altogether, our results demonstrated that Plexin-B2-deficient hESCs formed less compact and less cohesive colonies, whereas Plexin-B2 OE hESCs formed more adhesive colonies. As both Plexin-B2 KO and $\mathrm{OE}$ colonies displayed marked cellular disarray, it indicated that a balanced level of Plexin-B2 activity is required to orchestrate multicellular dynamics.

Plexin-B2 engages extracellular and Ras-GAP domains to orchestrate cytoskeletal network. Plexin-B2 can potentially 

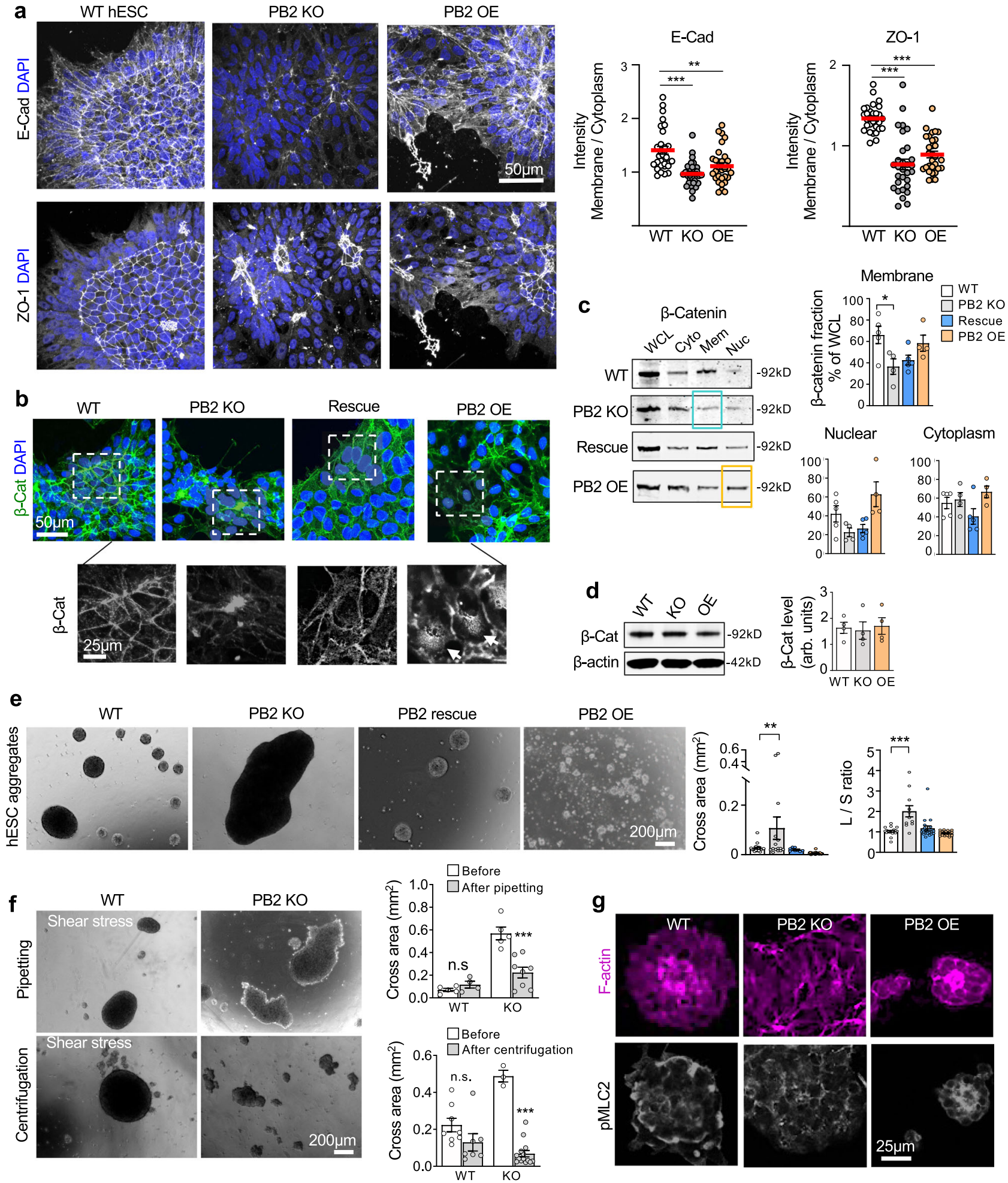

signal through its Ras-GAP domain to inactivate Ras/Rho small GTPases $^{56-59}$, its Rho-binding domain (RBD) that can bind to Rac or Rnd ${ }^{57,60}$, or its C-terminal PDZ binding motif as a docking site for PDZ-Rho-GEFs to activate RhoA ${ }^{61}$ (Fig. 6a). We next dissected which of these Plexin-B2 domains are engaged in the mechanoregulation of multicellular dynamics.

We introduced a series of Plexin-B2 mutants into KO hESCs to conduct domain-specific rescue experiments: Plexin-B2-mGAP (mutation of amino acids required for Ras-GAP activity), -mRBD (critical mutations in RBD domain), $-\triangle \mathrm{VTDL}$ (deletion of PDZ binding motif), and $-\triangle \mathrm{ECTO}$ (deletion of an extracellular domain) (Fig. 6a). WB and ICC confirmed comparable expression levels of Plexin-B2 signaling mutants in hESCs (Fig. S7a, b).

We found that the Plexin-B2 KO phenotypes-i.e., altered colony size and geometry, disorganized actomyosin and $\beta$-catenin networks-could be fully restored by wild-type (WT) Plexin-B2 rescue vector, largely rescued by Plexin-B2- $\triangle$ VTDL, partially rescued by Plexin-B2-mRBD, but minimally by Plexin-B2-mGAP and $-\triangle \mathrm{ECTO}$ (Fig. $6 \mathrm{~b}$ ). Hence, the extracellular and the Ras-GAP domains, and to a lesser degree RBD domain, are required for 
Fig. 4 Plexin-B2 influences cell-cell adhesion in multicellular organization of hESCs. a Confocal IF images show altered junction recruitment of E-cadherin and ZO-1 in mutant hESC colonies in contrast to the organized pattern in WT colony. Data points in quantification shown on right represent boundary/cytoplasm ratios of E-cadherin and ZO-1 immunointensities. $n=30$ cells per group. One-way ANOVA followed by Dunnett's multiple comparisons test. ${ }^{\star \star} P=0.0016$ and ${ }^{\star \star \star} P<0.0001$. Red lines represent mean values. $\mathbf{b}$ Confocal IF images reveal redistribution of $\beta$-catenin in mutant hESC colonies. Enlarged images of boxed areas are shown in bottom panels, highlighting $\beta$-catenin membrane localization in WT cells, which was shifted to the center of cell clusters in PLXNB2 KO. Arrows point to increased nuclear $\beta$-catenin in PLXNB2 OE cells. c WBs of cell fractions show a shift of membraneassociated (Mem) $\beta$-catenin to the cytoplasm (Cyto) in PLXNB2 KO hESCs (highlighted by blue box), which was rescued by CRISPR-resistant Plexin-B2. PLXNB2 OE resulted in increased nuclear (Nuc) $\beta$-catenin (highlighted by orange box). WCL whole cell lysate. Quantifications are shown to the right. Oneway ANOVA followed by Tukey's multiple comparisons test. $n=5$ for WT, $n=4$ for $P L X N B 2$ KO, $n=5$ for Rescue, and $n=4$ for $P L X N B 2$ OE. ${ }^{\star} P=0.0183$. Data represent mean \pm SEM. $\mathbf{d}$ WB and quantification show comparable levels of total $\beta$-catenin in WT and PLXNB2 mutant hESCs. $\beta$-actin served as a loading control. arb.units arbitrary units. One-way ANOVA followed by Tukey's multiple comparisons test. $n=4$ samples per group. Data represent mean \pm SEM. e Floating 3D hESC aggregates after $48 \mathrm{~h}$ culture in low adherence conditions. Quantifications of cross area and shape (L/S ratio, longest vs. shortest diameter) of aggregates are shown on the right. PB2 KO clone \#1. One-way ANOVA followed by Dunnett's multiple comparisons test. For cross area, $n=20$ aggregates for WT, $n=15$ for PLXNB2 KO, $n=9$ for Rescue, and $n=37$ for PLXNB2 OE. For L/S ratio, $n=19$ aggregates for WT, $n=11$ for $P L X N B 2$ KO, $n=19$ for Rescue, and $n=28$ for PLXNB2 OE from 3-5 fields. ${ }^{\star \star} P=0.0071,{ }^{\star \star \star}{ }^{*}<0.0001$. Data represent mean \pm SEM. $\mathbf{f} I m a g e s$ and quantifications show the reduced capability of PLXNB2 KO hESC aggregates to withstand shear forces applied by pipetting or low-speed centrifugation compared to WT. Two-sided unpaired $t$-test to compare within the groups. For pipetting: WT, $n=5$ aggregates before and $n=4$ after; $P L X N B 2 \mathrm{KO}, n=5$ before and $n=8$ after. ${ }^{\star \star \star} P=0.0008$. For centrifugation: WT, $n=8$ before and $n=7$ after; PLXNB2 KO, $n=3$ aggregates before and $n=14$ after.

${ }^{\star \star \star} P<0.0001$; n.s. not significant. Data represent mean \pm SEM. $\mathbf{g}$ IHC images of cross section of 3D hESC aggregates show that $P L X N B 2 \mathrm{KO}$ resulted in cellular disarray and disorganized actomyosin networks, whereas PLXNB2 OE led to enhanced actomyosin compact.

Plexin-B2 mechanoregulatory activity. In contrast, the C-terminal PDZ binding motif appeared dispensable, echoing the results from Rho-GEF inhibitor Y16 (see Fig. S6c, d),

Since the extracellular domain of Plexin-B2 is required to rescue the $\mathrm{KO}$ phenotype in the hESC colony, we tested how hESCs respond to Sema4C, a ligand for Plexin-B2. When WT hESCs were exposed to increasing concentrations of Sema4C mixed with Matrigel substrate, colony geometry was perturbed, displaying a contractile appearance with irregular contours, resembling the tensed appearance of PLXNB2 OE colonies (Fig. S7c). PLXNB2 OE cells responded to Sema4C similarly as WT cells, but Plexin-B2 KO cells appeared insensitive to Sema4C. Of note, anchorage of Sema4C to the cell membrane or substrate surface seemed critical as applying Sema4C in culture media did not elicit such an effect (Fig. S7d). It is notable that the effect of Sema4C was not identical to Plexin-B2 OE, thus a ligandindependent function of Plexin-B2 or its involvement in homotypic cell-cell adhesion remains a possibility ${ }^{18}$.

Plexin-B2 signals through RAP1/2 for mechanoregulation. Next, to validate the importance of the Ras-GAP domain of Plexin-B2 during hESC colony formation, we utilized genetic FRET probes to probe the activation status of RAP $1 / 2$ by live-cell imaging. Indeed, the FRET probes revealed that PLXNB2 OE cells showed reduced RAP1A and $2 \mathrm{~A}$ activation as compared to WT cells, while KO cells displayed enhanced RAP1/2 activation (Figs. 6c and S8a).

To further test the functions of RAP $1 / 2$ as downstream effectors of Plexin-B2, we introduced constitutively active (CA) or dominant-negative (DN) isoforms of RAP1B or $2 \mathrm{~A}$ into hESCs (Figs. 6d and S8b). In all three groups (WT, PLXNB2 KO, or OE), expression of CA RAP1B or $2 \mathrm{~A}$ phenocopied Plexin-B2 $\mathrm{KO}$ in regards to disorganized actomyosin networks, dissipation of the peripheral F-actin band, and filopodia-like protrusions at colony edge. Conversely, DN RAP1/2 phenocopied Plexin-B2 OE in regards to strengthened cortical F-actin, angular morphology, stretched cell borders, and spiky colony contours in all three genotypes, including Plexin-B2 KO (Figs. 6d and S8b). Additionally, membrane clustering of $\beta$-catenin, as well as the proliferation of hESCs, were affected by CA- or DN- Rap $1 / 2$ mutants, again phenocopying Plexin-B2 KO or OE, respectively (Fig. S8c, d). Hence, functional data support the engagement of
RAP1/2 by Plexin-B2 in the mechanoregulation of the hESC colony.

Plexin-B2-mediated cytoskeletal tension impacts on YAP/TAZ activity. Cells can respond to the mechanical properties of the surrounding physical environment, such as substrate rigidity, through the mechanosensitive transcription factors $\mathrm{YAP} / \mathrm{TAZ}^{36}$, which are the main executors of the Hippo pathway ${ }^{37}$. Of note, our transcriptomic analysis had indicated an effect of Plexin-B2 manipulations on Hippo/YAP/TAZ signaling (see Fig. S2a). To explore whether Plexin-B2 affects YAP/TAZ activation in hESC colonies, we examined the nuclear localization of YAP/TAZ as a readout-i.e., inactive YAP is phosphorylated and retained in the cytoplasm for degradation, whereas active YAP is translocated into the nucleus to drive pro-growth gene programs ${ }^{62,63}$. IF revealed a nucleus-to-cytoplasm shift of YAP/TAZ in Plexin-B2deficient hESCs as compared to WT, and conversely, a cytoplasm-to-nucleus shift in OE cells (Figs. 7a and S9a). It is worth noting that, unlike differentiated cells, human PSCs are insensitive to contact inhibition as they display a sustained basal YAP-driven transcriptional activity despite growing as dense colonies $^{64}$.

Cytoskeletal mechanotension ensured by Plexin-B2 signaling may in fact facilitate response to extracellular mechanical cues such as substrate stiffness. To address this, we examined YAP activation in WT or Plexin-B2 mutant hESCs grown on soft or stiff substrates. Results showed that WT and Plexin-B2 OE hESCs responded to softer substrates with reduced nuclear YAP and to a stiffer substrate with YAP nuclear translocation, with Plexin-B2 OE cells responded more robustly than WT cells with higher levels of nuclear YAP (Fig. 7a). In contrast, Plexin-B2 KO hESCs were insensitive to substrates stiffness in terms of YAP nuclear localization, which remained low in all conditions.

Consistently, WB showed higher levels of YAP in PLXNB2 OE hESCs than in WT (Fig. 7b). Furthermore, the expression of YAP target genes CYR61, CTGF, and ANKRD1 was robustly upregulated in response to Dox-induced overexpression of Plexin-B2 in hESCs (Fig. 7c). Our RNA-seq data also showed transcriptional changes of YAP signature genes in Plexin-B2 mutant hESCs as compared to WT, and principal component analysis (PCA) based on the expression of YAP signature genes showed clear segregation of WT, PLXNB2 KO, and OE hESC samples, confirming that each genotype leads to a distinct state of 
a
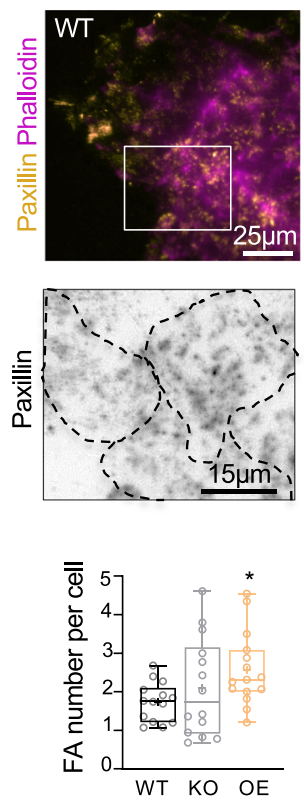

hESC TIRF
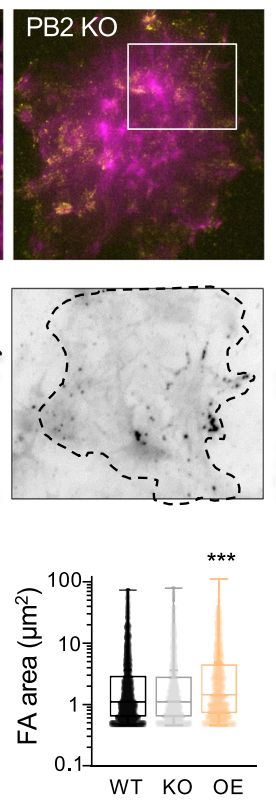

b
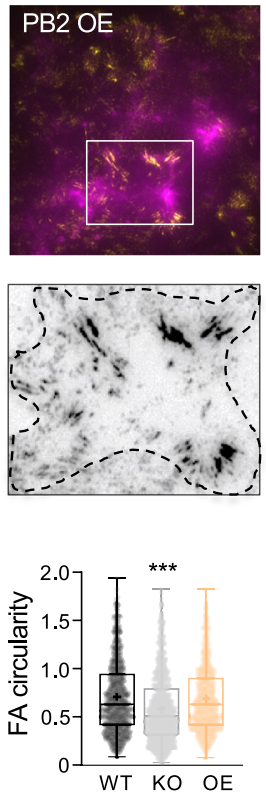

W
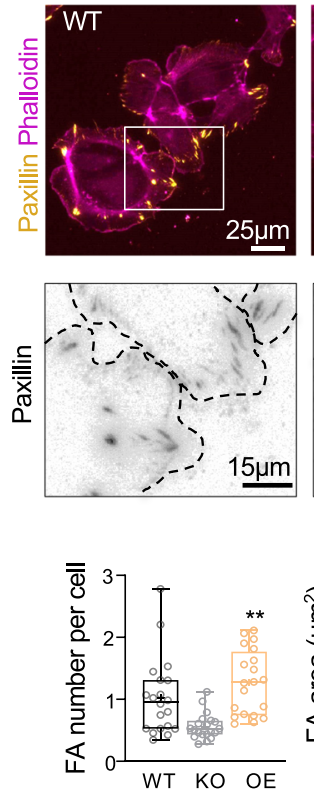

hNPC TIRF
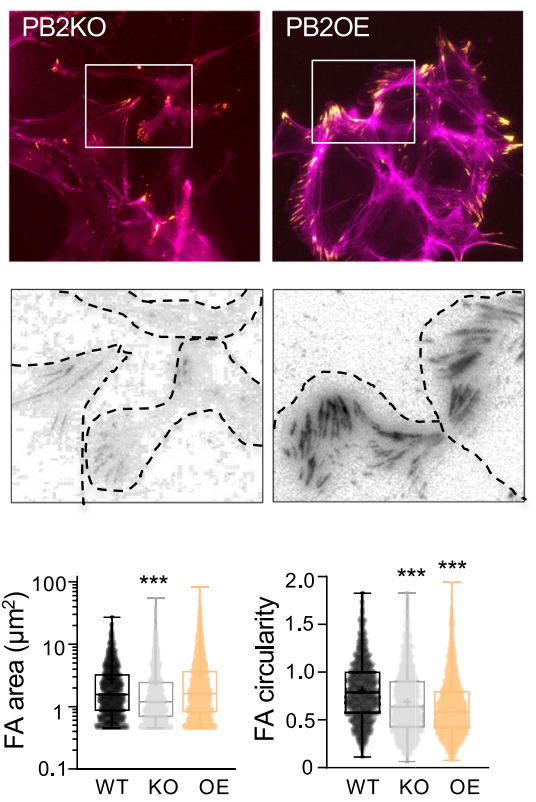
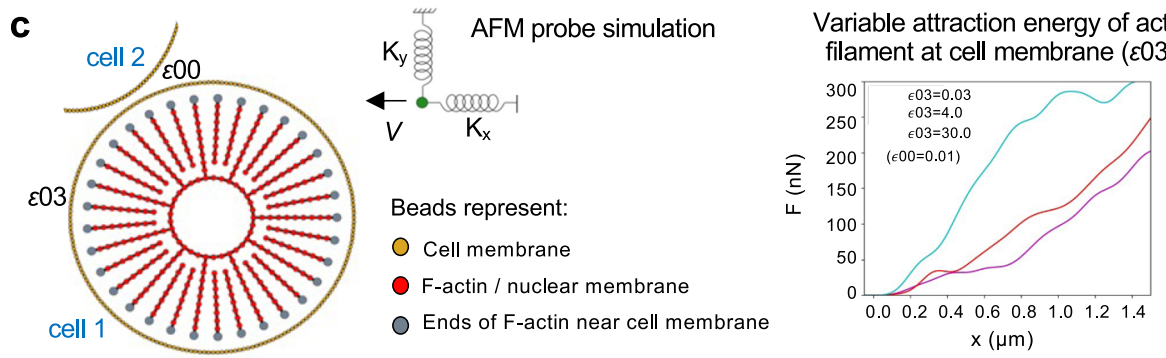

Variable membrane-membrane attraction energy $(\varepsilon 00)$

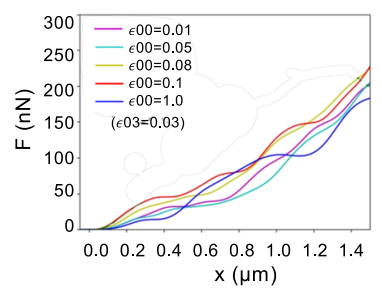

CellMask

d
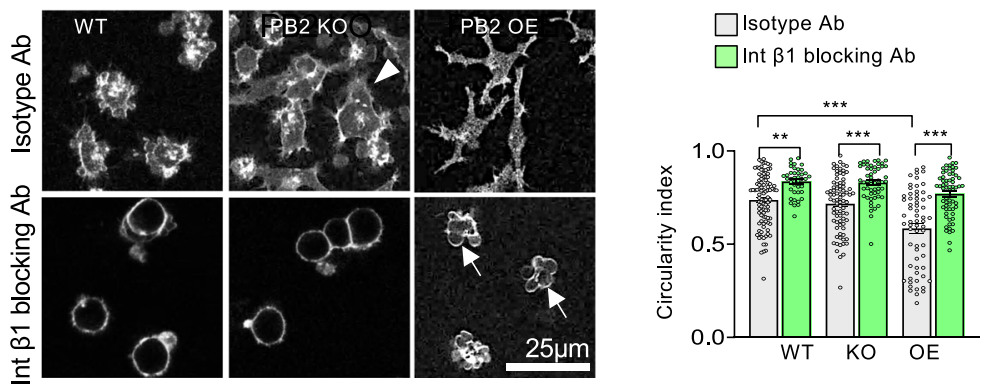

Fig. 5 Plexin-B2 influences cell-matrix adhesion and controls contractility in hESC colonies. a, b TIRF microscopy images and quantification show altered number, size, and shape of focal adhesions (FAs) in PLXNB2 mutant hESC colonies (a) and hNPCs (b) compared to wild-type (WT) cells. Cells were stained for FA marker paxillin and F-actin (phalloidin). Enlarged images of boxed areas are shown below in inverted black and white, with dashed lines outlining individual cells. Box plots depict 25-75\% quantiles, minimal and maximal values (whiskers), median (midline), and mean (cross signs). For hESCs: FA number, $n=14-15$ cells per group. FA area, $n=2271$ FAs for WT, $n=1042$ for PB2 KO, and $n=978$ for PB2 OE. FA circularity, $n=1042$ FAs for WT, $n=1042$ for PB2 KO, and $n=978$ for PB2 OE. ${ }^{\star} P=0.0397$, ${ }^{\star \star \star} P<0.0001$. For hNPCs: FA number, $n=20$ cells per group. FA area and circularity, $n=1829$ FAs for WT, $n=2385$ for PB2 KO, and $n=4528$ for PB2 OE. Kruskal-Wallis test followed by Dunn's multiple comparisons test. ${ }^{\star \star} P=0.0084$,

${ }^{\star \star \star} P<0.0001$. c Molecular dynamics mathematical simulation. Left, components of simulation based on a bead-spring model. The tip of the simulated AFM probe is depicted as a green sphere, with elastic constants $K_{x}$ and $K_{y}$ in the $x$ and $y$ directions, respectively. Right, graphs of force as a function of distance traveled by the simulated AFM probe in two different scenarios. Left, when varying the actin-membrane attraction energy that simulates increasing contractility, the response of the force to the virtual AFM probe resembles the results of the experimental AFM data in accordance to Plexin-B2 activity (see Fig. 2). Such result is not seen when varying membrane-membrane attraction energy (right). d Left, experimental design for live-cell imaging. Middle, images of hESCs labeled with CellMask reveal distinct cell morphology when exposed to a function-blocking antibody against integrin $\beta 1$ (P5D2) or an isotype control lgG. Arrowhead points to cellular protrusion from PLXNB2 KO hESCs. Arrows point to cell membrane blebbing of PLXNB2 OE hESCs after integrin blockade. Quantification for cell roundness (circularity index) is shown on right. One-way ANOVA followed by Tukey's multiple comparisons test. For isotype lgG treatment: $n=100$ cells for WT, $n=80$ cells for PB2 KO, and $n=63$ for PB2 OE. For integrin $\beta 1$ treatment: $n=41$ cells for WT, $n=54$ for $\mathrm{PB} 2 \mathrm{KO}$, and $n=63$ cells for PB2 OE. ${ }^{\star \star} P=0.0025$ and ${ }^{\star \star \star} P<0.0001$. Data represent mean $\pm \mathrm{SEM}$. 
a

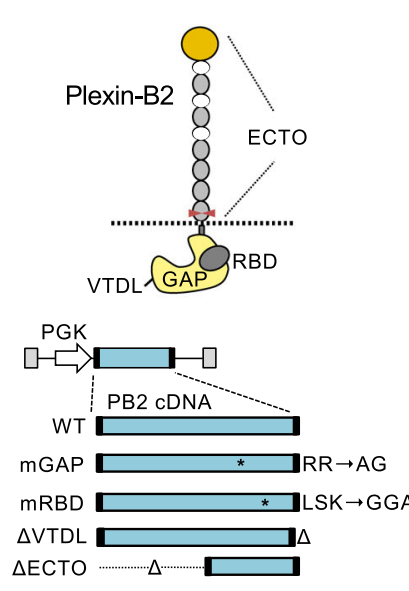

C

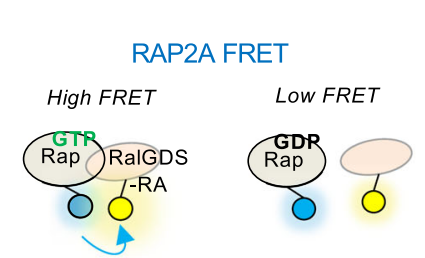

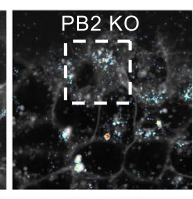
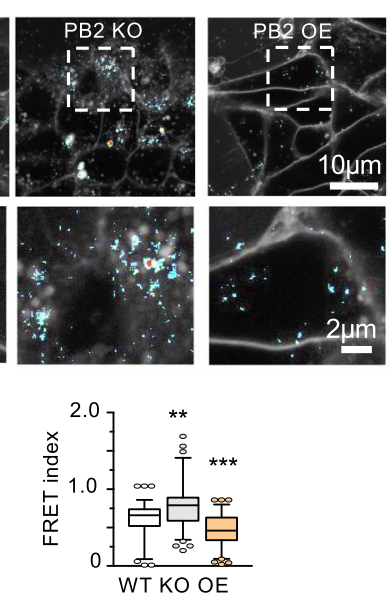

PB2-Rescue
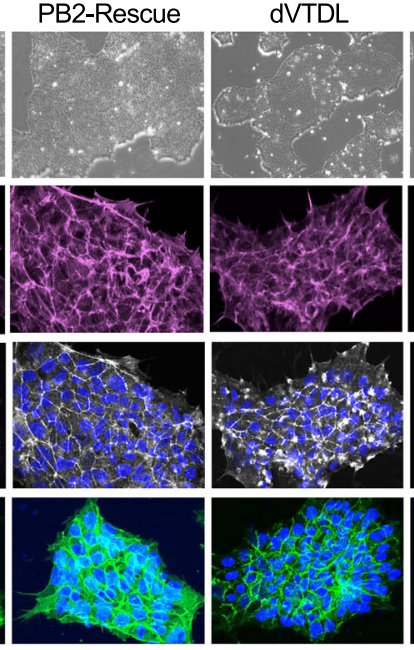

Low
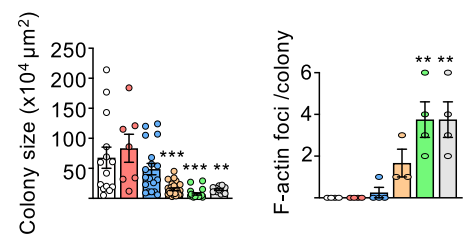

d

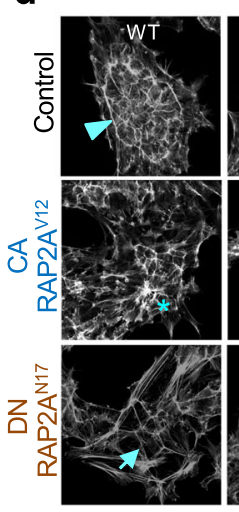

F-actin
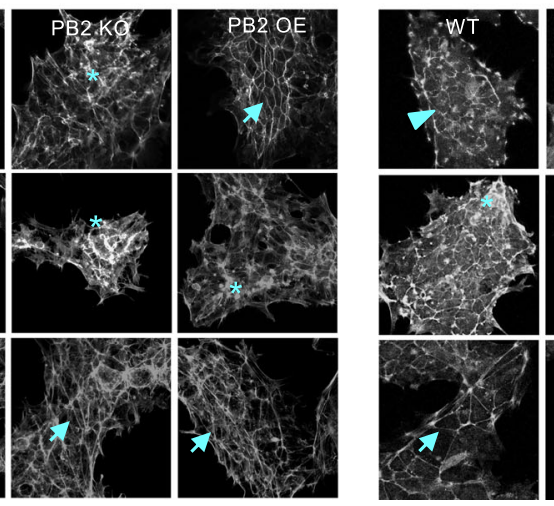

pMLC2
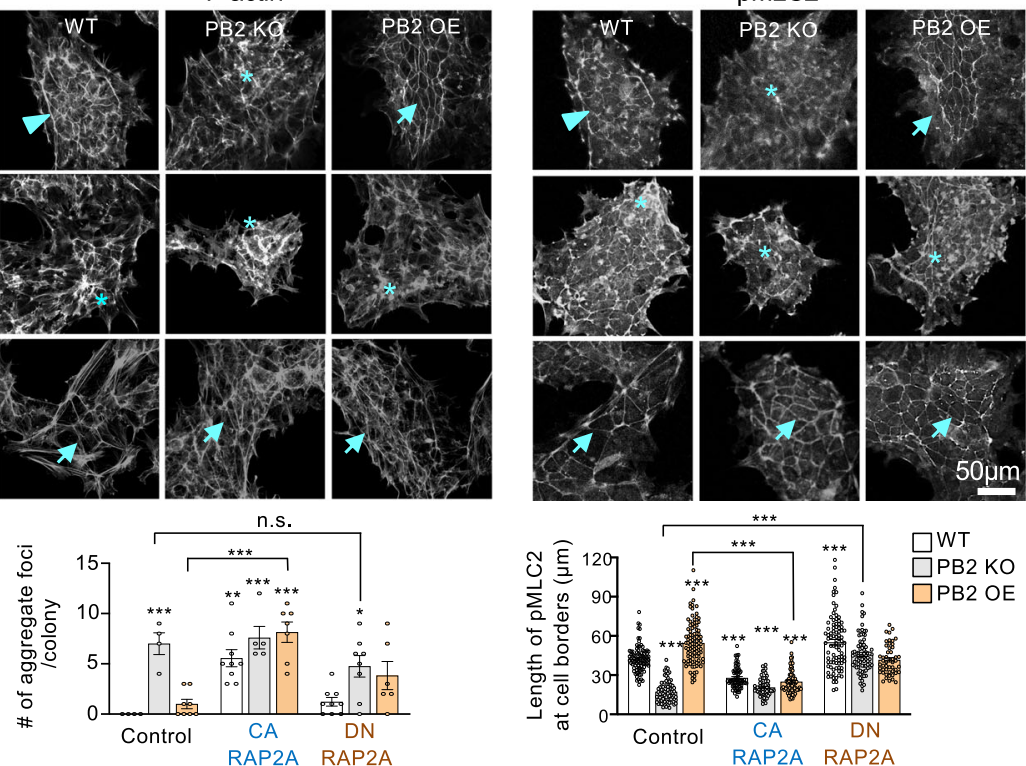

$\mathrm{mRBD}$
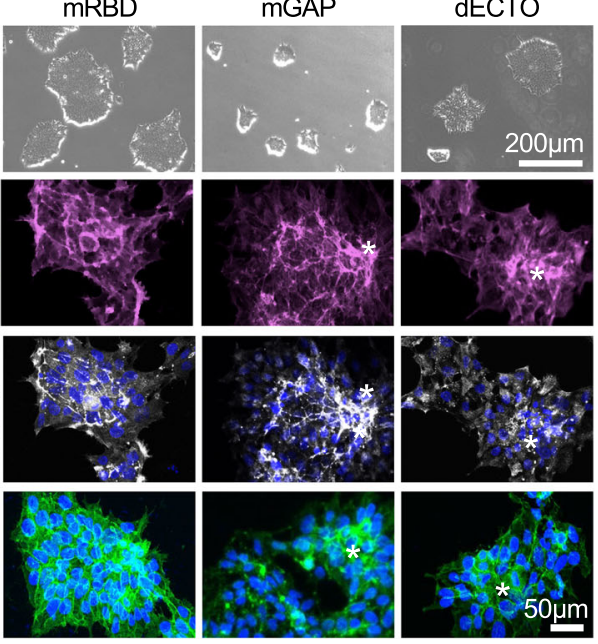

High
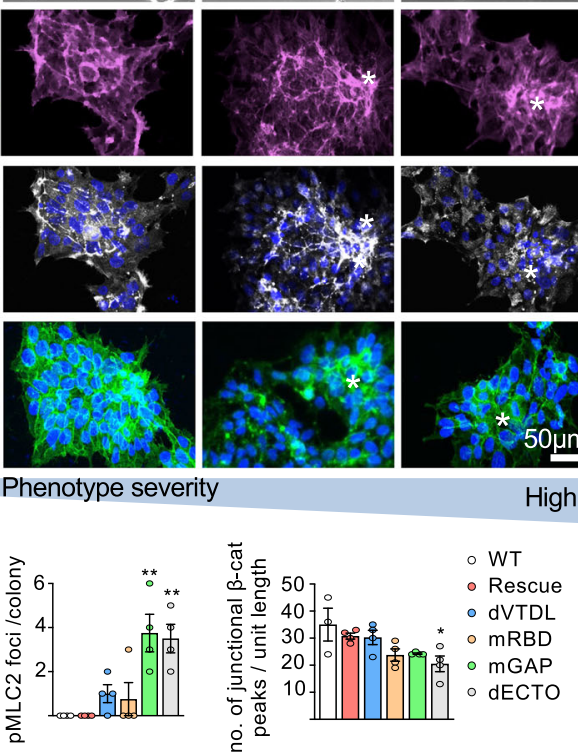

RAP2A RAP2A

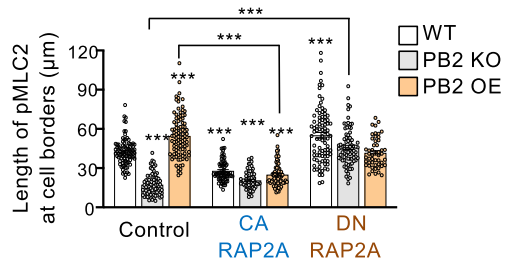

index

YAP signaling (Fig. S9b, c). Interestingly some of the YAP target genes responded differently to constitutive overexpression vs. acute upregulation of Plexin-B2 in the inducible model (Fig. S9b). This may be reconciled by the fact that certain YAP target genes have been shown to act as a transient response to biomechanical stimuli 65 .

Additionally, the introduction of CA RAP2A resulted in YAP inactivation (nucleus-to-cytoplasm shift) in all three genotypes, phenocopying Plexin-B2 KO, whereas DN RAP2A reverted YAP inactivation in Plexin-B2 KO cells (Fig. 7d).

To further interrogate a functional link between Plexin-B2 and YAP signaling, we introduced a Dox-inducible CA form of YAP (YAP1 ${ }^{5 S A}$ ) into WT or PLXNB2 mutant hESCs (Fig. S9d), which led to angular cell morphologies and strengthened cortical F-actin in WT but also in PLXNB2 KO hESCs, reminiscent of the OE phenotypes (Fig. 7e). Conversely, YAP1 KD by shRNA resulted in diminished cortical F-actin in WT hESCs, but also attenuated the hypercontractile phenotype of Plexin-B2 OE cells (Figs. 7f and S9e, f).

As another way to test the functional relationship of Plexin-B2 and YAP and $\beta$-catenin in hESCs, we exposed hESCs to verteporfin (VP), an inhibitor of YAP signaling, or cardamonin, which promotes degradation of $\beta$-catenin (Fig. S10a). Results showed that VP markedly reduced proliferation of WT and PLXNB2 OE hESCs, but did not further diminish the proliferation of KO cells (Fig. S10b), thus supporting YAP as a downstream effector of Plexin-B2 in regard to hESC proliferation. Correspondingly, the colony size was reduced in WT and PlexinB2 OE by VP, but VP did not further reduce the size of PLXNB2 KO colonies, which were already smaller than WT (Fig. S10b). 
Fig. 6 Plexin-B2 signals through RAP1/2 to orchestrate cell mechanics in hESC colonies. a Top, Plexin-B2 domain structure: ECTO ectodomain, RBD Rho-binding domain, GAP Ras-GTPase domain, VTDL, four C-terminal amino acids, forming PDZ binding motif. The cleavage site of mature Plexin-B2 is depicted by red arrows. Bottom, lentiviral vectors expressing wild-type (WT) Plexin-B2 or signaling mutants (CRISPR-KO resistant): mGAP mutation in GAP, mRBD mutation in RBD, $\triangle$ VTDL deletion of VTDL, $\triangle E C T O$ deletion of ECTO domain. b Phase-contrast and confocal images illustrate the severity of cytoskeletal disarray and $\beta$-catenin membrane distribution phenotypes of hESC colonies expressing wild-type or Plexin-B2 signaling mutants (in the background of PLXNB2 KO). Asterisks denote abnormal cell clusters within the colony. Quantifications are shown below. One-way ANOVA followed by Tukey's multiple comparisons test. For hESC colony size: $n=14$ colonies for WT, $n=7$ for Rescue, $n=18$ for dVTDL, $n=24$ for mRBD, $n=21$ for mGAP, and $n=12$ for dECTO. ${ }^{\star \star} P=0.0028 ;{ }^{\star \star \star} P<0.0003$ (WT vs mRBD), ${ }^{\star \star \star} P<0.0001$ (WT vs mGAP). For others, $n=3-4$ for each group. For F-actin foci, ${ }^{\star \star} P=0.002$. For $p M L C 2$ foci, ${ }^{\star \star} P=0.0019$ (WT vs. mGAP) and ${ }^{\star \star} P=0.0037$ (WT vs. dECTO). For junctional $\beta$-cat peaks, ${ }^{\star} P=0.025$. Data represent mean \pm SEM. c Top, diagram of FRET probes for RAP2A activation status. Middle, representative images of normalized RAP2A FRET signals in WT, PLXNB2 KO (clone \#3), PLXNB2 OE hESCs. Enlarged images of boxed areas are shown below. Box plots show median, 25-75\% quantiles, and top or bottom 5\% data points. Kruskal-Wallis test followed by Dunn's multiple comparisons test. $n=98$ cell-cell membrane areas for WT; $n=89$ for PB2 KO; $n=85$ for $P L X N B 2$ OE. ${ }^{\star \star} P=0.0013$ and ${ }^{\star \star \star} P<0.0001$. d Confocal IF images show the effects of RAP2A isoforms on cortical actomyosin network, colony geometry, and cellular organization of hESC colonies as compared to control colonies with no RAP2A expression vector. Arrowheads point to the peripheral F-actin band in WT colony, asterisks denote abnormal cell cluster in PLXNB2 KO colony, and arrows point to tensed cortical F-actin and stretched junction borders in PLXNB2 OE colony. Note that constitutively active (CA) RAP2AV12 phenocopied Plexin-B2 KO (clone \#3), whereas dominant-negative (DN) RAP2AN17 phenocopied Plexin-B2 OE. Quantifications of actomyosin aggregate foci per colony and length of pMLC2 at cell borders are shown below. For aggregate foci: control, $n=4$ colonies for WT, $n=4$ for PB2 KO, and $n=8$ for PB2 OE; CA RAP2AV12, $n=9$ for WT, $n=5$ for PB2 KO, and $n=7$ for PB2 OE; DN RAP2A ${ }^{N 17}, n=9$ for WT, $n=8$ for PB2 KO, and $n=6$ for PB2 OE. ${ }^{\star} P=0.010,{ }^{\star} P=0.0017,{ }^{\star \star \star} P<0.0001$. For pMLC2: control, $n=80-100$ cell borders for each genotype; CA RAP2AV12, $n=60-70$ for each genotype; DN RAP2AN17, WT, $n=50-90$ for each genotype. ${ }^{\star \star *} P<0.0001$. One-way ANOVA followed by Dunnett's post hoc correction to compare to WT group. A two-sided unpaired t-test was applied when comparing data from two groups (brackets). Data represent mean \pm SEM.

$\mathrm{VP}$ also phenocopied YAP1 KD in reducing cortical F-actin in WT or Plexin-B2 OE hESCs, and attenuated the hypercontractile appearance of OE cells (Fig. S10c). In contrast, cardamonin did not significantly alter the proliferation or cortical F-actin patterns in hESCs of either of the three genotypes, but it altered cellular arrangement in WT hESC colonies (Fig. S10b, c). These observations fit with our mathematical simulations that PlexinB2 primarily controls actomyosin contractility, with secondary effects on adhesion complexes during the multicellular organization.

Hence, YAP can not only respond to Plexin-B2-mediated cytoskeletal tension but can mediate cytoskeletal dynamics and stem cell state in hESC colonies. Moreover, Plexin-B2-mediated cytoskeletal tension ensures mechanoresponse of YAP to extracellular mechanical cues.

Altered biomechanics affects ERK1/2 activation, calcium distribution, and stem cell physiology in hESC colony. We next investigated the effect of Plexin-B2 on stem cell state. Comparative analysis of differentially engaged signaling pathways in PLXNB2 KO or OE hESCs relative to WT indicated activation of ERK/MAPK and calcium signaling (Fig. 7g). We, therefore, assessed ERK1/2 activation by WB, which indeed showed elevated levels of phosphorylated ERK1/2 in PLXNB2 OE hESCs relative to WT or KO cells (Figs. 7h and S10d). Again, the effect of PlexinB2 OE on ERK1/2 activation could be reverted by expression of CA RAP1/2; and conversely, expression of DN RAP1/2 led to a marked increase of ERK1/2 activation in all three genotypes (Figs. 7h and S10d), further supporting RAP1/2 as a downstream effector of Plexin-B2 in this regard.

We next performed live calcium imaging of hESC colonies, which revealed a distinct pattern of cytosolic calcium levelshigher at colony periphery and lower in colony interior-in agreement with the higher traction forces at colony edges 6 (Fig. 7i). Notably, PLXNB2 $\mathrm{KO}$ and OE disrupted the spatial pattern of calcium within the colonies, echoing the disarray of actomyosin networks. Furthermore, calcium signals were in general elevated in PLXNB2 OE cells (Fig. 7i).

Interestingly, the altered biomechanical properties of hESC colonies from Plexin-B2 manipulations also affected the orientation of mitotic spindles at the colony edge. In WT and PLXNB2
OE colonies, the cell division planes at the colony periphery were largely oriented perpendicular to the colony rim, in alignment with in-plane traction forces, but they were randomly oriented at the colony center (Fig. S11). In contrast, cell division planes in $\mathrm{KO}$ colonies were largely randomized in all areas (Fig. S11). Hence, distinct mechanical niches as orchestrated by Plexin-B2 govern mitotic spindle orientation in hESC colonies.

Colony integrity is an indicator of the stemness state of hESCs $^{8}$. Although hESCs continued to express the pluripotency factors SOX2, OCT4, and NANOG in PLXNB2 KO or OE colonies (Fig. S12a), we found that Plexin-B2-deficient colonies harbored small patches of cells expressing neural stem cell marker PAX6, indicating premature neural lineage differentiation (Figs. 8a and S12b). By comparison, Plexin-B2 OE did not cause premature induction of PAX6. Indeed, our RNA-seq data confirmed that PLXNB2 KO hESCs upregulated genes related to ectoderm differentiation, dopamine neurogenesis, and neural crest differentiation, indicative of premature neuronal differentiation in $\mathrm{KO}$ colonies, while PLXNB2 OE cells upregulated genes related to mesodermal commitment and cardiac progenitor differentiation (see Fig. S2a).

For corroboration of premature lineage commitment in PLXNB2 KO hESCs, we carried out qRT-PCR array assays on the expression of a set of stem cell and differentiation markers. Around $90 \%$ of these markers exhibited no significant transcriptional alteration in PLXNB2 KO relative to WT hESCs, including ESC markers OCT4, NANOG, and SOX2 (Fig. S12c). Of the remaining $10 \%$ markers that showed transcriptional changes, notably all displayed upregulation in PLXNB2 KO cells, including markers for the three germ layers: i.e., GSC (endoderm), DES (mesoderm), as well as neural markers, e.g., GFAP and SOX8 (Fig. S12c), which were further confirmed by qRT-PCR (Fig. 8b).

Plexin-B2 affects the proliferation and differentiation of hNPCs and neuroepithelial cytoarchitecture. We next studied the effect of Plexin-B2 on hNPC biology. We found that as in hESCs, PLXNB2 KO also resulted in reduced proliferation of hNPCs, while PLXNB2 OE did not further enhance the already high proliferative state of hNPCs (Fig. S13a).

Upon neural induction of hESCs, Plexin-B2 deficient cells consistently displayed accelerated neuronal differentiation 
a
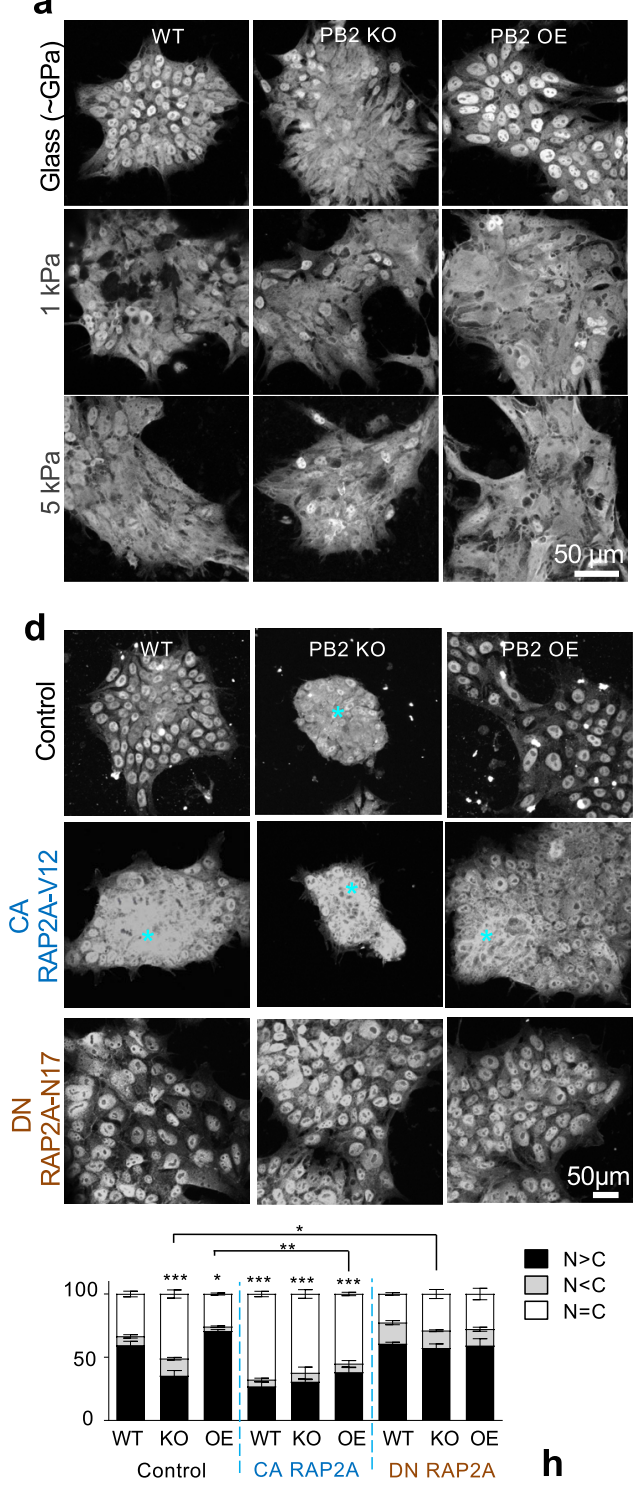

g

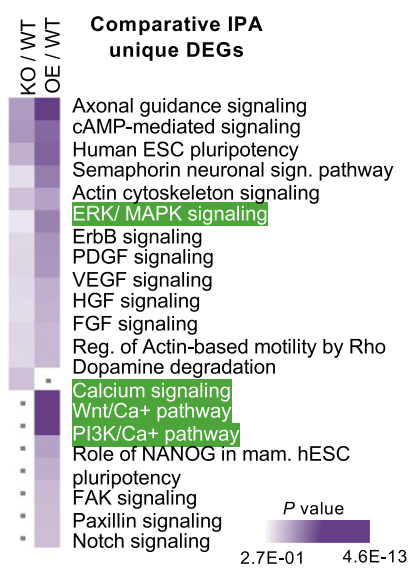

$\mathrm{N}>\mathrm{C}$

$\square \begin{aligned} & \mathrm{N}<\mathrm{C} \\ & \mathrm{N}=\mathrm{C}\end{aligned}$

h

\section{i}

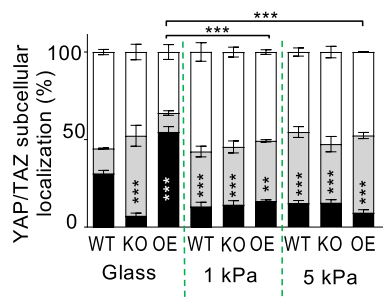

C

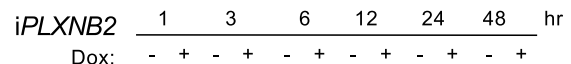

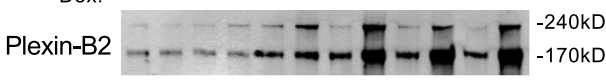

GAPDH -

$\mathrm{N}>\mathrm{C}$
$\mathrm{N}<\mathrm{C}$
$\mathrm{N}=\mathrm{C}$
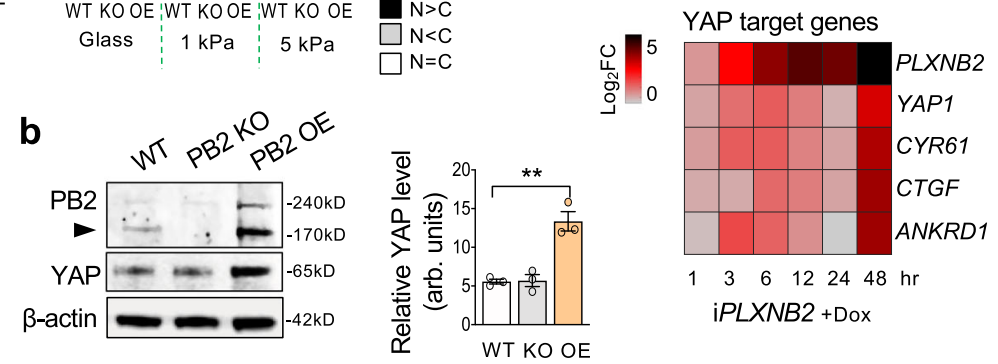

e
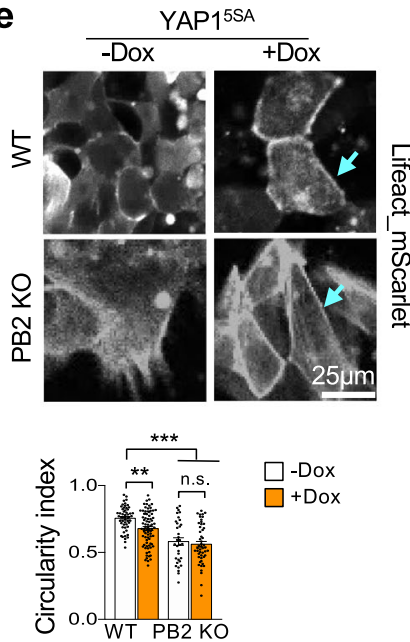

$\square$-Dox
$\square+$ Dox f
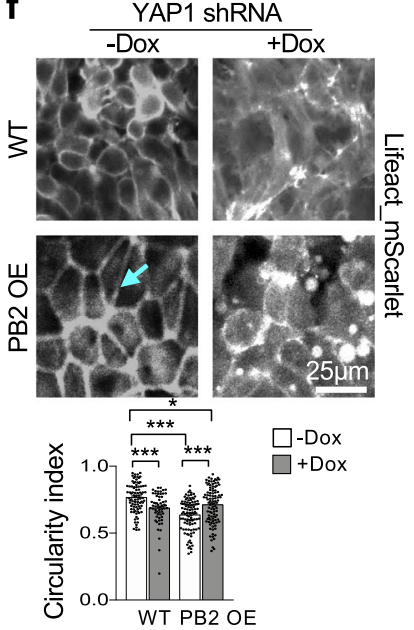

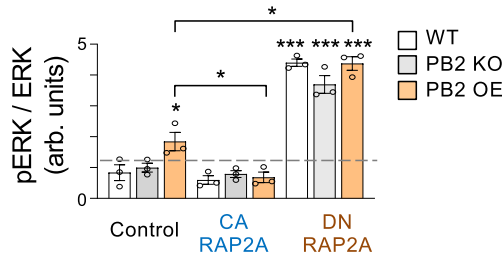

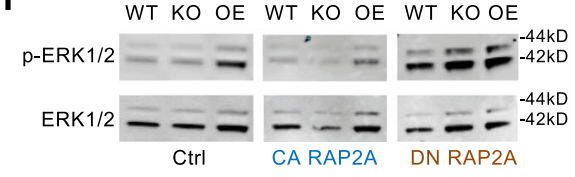
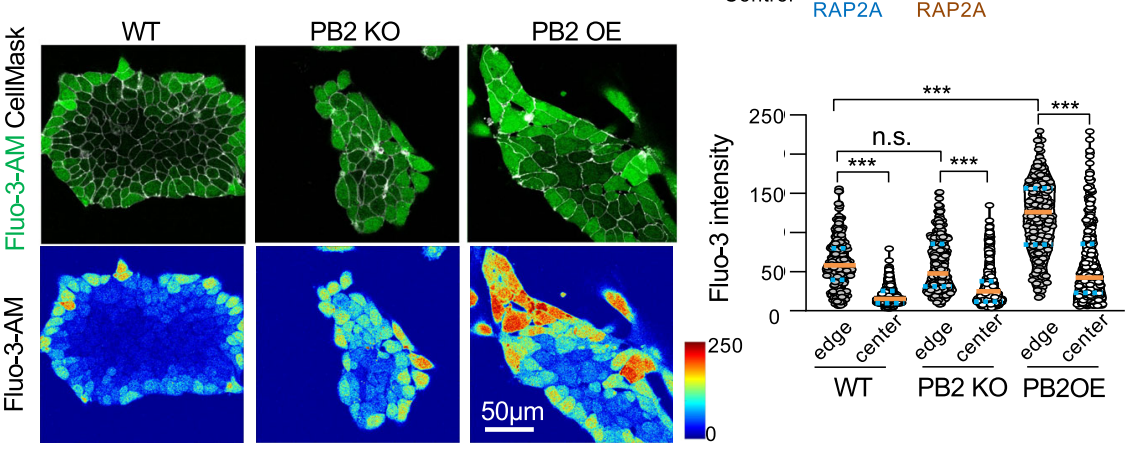

compared to WT counterparts, as evidenced by enhanced induction of the neuroblast marker doublecortin (DCX) and the neuronal marker $\beta$-III tubulin, and reduced expression of NPC markers PAX6 and SOX2 (Figs. 8c and S13b, c). Analyses by qRT-PCR confirmed upregulation of TUBB3 (encoding $\beta$-III tubulin) in PLXNB2 KO cells, whereas PLXNB2 OE did not cause accelerated neuronal differentiation (Fig. 8c).

In addition, WB also showed a response of YAP to Plexin-B2 manipulation in hNPCs, with higher pYAP levels (signifying inactivation) in PLXNB2 KO cells, but elevated YAP levels in OE cells (Fig. 8d). We further tested the effect of $\beta$-catenin or YAP 
Fig. 7 Plexin-B2 engages the YAP mechanotransduction pathway and activates ERK1/2 and calcium signaling. a Confocal IF images show reduced nuclear YAP/TAZ in PLXNB2 KO hESCs, but a cytoplasm-to-nucleus shift in PLXNB2 OE as compared to WT cells on standard cell culture substrate (glass coverslip coated with Matrigel). Cultures on hydrogels with softer stiffness ( 1 and $5 \mathrm{kPa}$ ) is similar for all genotype groups with a strong reduction of nuclear YAP/TAZ. Quantifications show relative fractions of cells with nuclear YAP/TAZ signals that are greater, equal, or smaller than cytoplasmic signals ( $N>C, N=C, N<C$ ). Two-way ANOVA followed by Dunnett's post hoc test to compare to WT. $n=115-301$ cells for each condition ${ }^{\star \star} P=0.0019$, ${ }^{\star \star \star} P=0.0001$ (WT glass vs $\mathrm{KO}$ and OE glass), ${ }^{\star \star \star} P=0.0002$ (WT glass vs WT $1 \mathrm{kPa}$ ), ${ }^{\star \star \star} P=0.0004$ (WT glass vs KO $1 \mathrm{kPa}$ ), ${ }^{\star \star \star} P=0.0008(\mathrm{WT}$ glass vs WT $5 \mathrm{kPa}$ ), ${ }^{\star \star \star} P=0.0009$ (WT glass vs $\mathrm{KO} 5 \mathrm{kPa}$ ), ${ }^{\star \star \star} P=0.0001$ (WT glass vs OE $5 \mathrm{kPa}$ ). A two-sided unpaired $t$-test was applied when comparing data from two groups (brackets). ${ }^{\star \star \star} P=0.0002$ (OE Glass vs OE $1 \mathrm{kPa}$, and OE Glass vs OE $5 \mathrm{kPa}$ ), Data represent mean $\pm \mathrm{SEM}$. b WB and quantification show an increased level of total YAP in PLXNB2 OE cells as compared to WT or PLXNB2 KO hESCs. Arrowhead points to mature Plexin-B2 band. $\beta$-actin served as a loading control. arb.units arbitrary units. One-way ANOVA followed by Tukey's multiple comparisons test. $n=3$ samples per group.

${ }^{\star \star} P=0.0017$. Data represent mean \pm SEM. c Top, WB shows time course of Dox-induced expression of Plexin-B2 (iPLXNB2) in hESCs. GAPDH served as a loading control. Bottom, heatmap of qRT-PCR data illustrates upregulation of YAP1 and its target genes CYR61, CTGF, and ANKRD1 upon Plexin-B2 induction. The heatmap was generated by the mean value from $n=3$ independent cultures. $\mathbf{d}$ IF images show the effects of RAP2A isoforms on YAP/TAZ nuclei/ cytoplasmic localization in hESC colonies. Note that expression of constitutive active RAP2AV12 led to elevated cytoplasmic YAP/TAZ in all three groups, phenocopying Plexin-B2 KO. Quantification shows relative fractions of cells with nuclear YAP/TAZ immunosignals that are greater, equal, or smaller than cytoplasmic signals ( $N>C, N=C, N<C$ ). Two-way ANOVA followed by Dunnett's post hoc test to compare to WT group. For control, $n=330-466$ cells for each genotype; for CA RAP2A V12, $n=163-233$ for each genotype; for DN RAP2A ${ }^{N 17}, n=412-543$ for each genotype ${ }^{\star} P=0.040$ and ${ }^{\star \star \star} P<0.0001$. A two-sided unpaired $t$-test was applied when comparing data from two groups (brackets). ${ }^{\star} P=0.015,{ }^{\star \star} P=0.0012$. Data represent mean $\pm \mathrm{SEM}$. e, $\mathbf{f}$ Livecell imaging shows differences in cortical F-actin (visualized by LifeAct_mScarlet) in hESCs with Dox-inducible expression of YAP15SA (e) or YAP1 shRNA (f). Arrows point to tensed cortical F-actin and stretched cell borders. Data represent mean \pm SEM. One-way ANOVA with Tukey's post hoc test. For YAP15SA: WT, $n=54-80$ cells; PB2 KO, $n=33-50$ cells. ${ }^{\star \star} P=0.0053$ and ${ }^{\star \star *} P<0.0001$. For YAP1 shRNA: WT, $n=62-89$ cells; PB2 OE, $n=100$ cells each. ${ }^{\star} P=0.012$ and ${ }^{\star \star \star} P<0.0001$. n.s. not significant. Data represent mean \pm SEM. $\mathbf{g}$ Comparative Ingenuity pathway analysis (IPA) of enriched gene pathways for the unique DEGs identified in PLXNB2 KO or OE hESCs relative to WT. Note the enhanced activation of ERK/MAPK and calcium signaling in Plexin-B2 OE cells. The categories are ranked by adjusted $P$ value computed by IPA's Fisher's Exact Test. $\mathbf{h}$ WBs show increased ERK1/2 phosphorylation ( $p$-ERK1/2) in PLXNB2 OE cells compared to WT. p-ERK1/2 was further increased by DN RAP2AN17 expression in all three groups (WT, PB2 KO, and PB2 OE). In contrast, CA RAP2AV12 expression resulted in reduced p-ERK1/2 in PLXNB2 OE cells. ERK1/2 served as a loading control. arb.units arbitrary units. One-way ANOVA followed by Dunnett's post hoc correction to compare to WT group. A two-sided unpaired $t$-test was applied when comparing data from two groups (brackets). $n=3$ samples per group. ${ }^{\star} P=0.017$ (WT vs. PB2 OE), ${ }^{\star} P=0.028$ (Control PB2 OE vs. CA RAP2AV12 PB2 OE), ${ }^{\star} P=0.0024$ (Control PB2 OE vs. DN RAP2AN17 PB2 OE), ${ }^{\star \star \star} P<0.0001$. Data represent mean \pm SEM. i Calcium imaging with Fluo-3-AM dye shows higher levels of intracellular calcium in hESCs at colony edges compared to central areas. Calcium signals were elevated in PLXNB2 OE. Kruskal-Wallis test followed by Dunn's multiple comparisons test. For colony edges, $n=328,332$, and 116 for WT, PB2 KO, and PB2 OE, respectively. For colony center, $n=1000,648$, 417 cells for WT, PB2 KO, and PB2 OE, respectively. ${ }^{\star \star \star} P<0.0001$. Orange lines indicate median values and blue lines 25 and $75 \%$ percentiles.

inhibitors on neural induction (Fig. S13d). For $\beta$-catenin inhibition with cardamonin, we observed no overt effects on neuronal differentiation in either of the three groups, with PLXNB2 KO cells similarly displaying enhanced neuronal differentiation with or without cardamonin exposure. For YAP inhibition with VP, we observed that limited VP exposure indeed promoted neuronal differentiation for WT and PLXNB2 OE cells, a phenotype similar to Plexin-B2 KO. These data provide additional support for a link of Plexin-B2 and YAP in controlling stem cell differentiation.

To further probe the role of Plexin-B2 in neuroepithelial tissue morphogenesis, we generated 3D cerebral organoids from hESCs using a forebrain organoid differentiation protocol ${ }^{66,67}$. After 6 weeks of culture, layered cortical structures emerged in cerebral organoids, with ventricular zones occupied by neuroprogenitors $\left(\mathrm{SOX}^{+}\right)$and cortical plate-like zones occupied by differentiating neuroblasts $\left(\mathrm{DCX}^{+}\right)$(Fig. 8e, f).

Cerebral organoids derived from PLXNB2 $\mathrm{KO}$ or OE hESCs were consistently smaller than WT organoids (Fig. 8e). Histological analysis of organoid cross sections revealed severe disruption of neuroepithelial cytoarchitecture in mutant organoids, e.g., absence of organized ventricular structures, which were replaced with numerous simple cysts in PLXNB2 KO organoids, and large cystic structures with stretched and irregular contours in $\mathrm{OE}$ organoids (Fig. 8f). Notably, both $\mathrm{SOX}^{+}$and $\mathrm{DCX}^{+}$cells were present in mutant organoids, albeit in spatial disarray (Fig. 8f). The disorganization of neuroepithelium was also reflected by the disarray of the actomyosin network, activated integrin $\beta 1$, pFAK, as well as junctional $\mathrm{N}$-cadherin, corresponding to Plexin-B2 manipulations (Fig. S13e). Thus, Plexin-B2 is not strictly required for neural induction per se, but affects cellular alignment and architectural integrity during neuroepithelial morphogenesis.

\section{Discussion}

The multicellular organization relies on force-mediated processes that orchestrate cytoskeletal organization across cells in order to keep individual cells in a certain shape while maintaining tissue cohesion and structural integrity ${ }^{68}$. The signaling pathways that transmit external guidance cues into cytoskeletal tension and collective formation of actomyosin network and adhesive complexes need to be better mapped.

Here, we provided multiple lines of evidence to support the role of the guidance receptor Plexin-B2 in orchestrating actomyosin cytoskeletal network and cell-cell adhesion during multicellular development of hESCs and hNPCs. This impacts signaling pathways of $\beta$-catenin, YAP, ERK1/2, and calcium, all critical for stem cell physiology (Fig. 8g). From a mechanical perspective, the correlation between the experimental AFM data and mathematical simulations indicated that Plexin-B2 primarily promotes actomyosin contractility, which in turn affects tensile forces exerted on cell-cell junctions and cell-matrix adhesion sites, underscoring the interconnectedness of these mechanical elements ${ }^{8,69,70}$. The fundamental mechanoregulatory function of Plexin-B2 is consistent with an early evolutionary appearance of semaphorins and plexins at the transition from unicellular to multicellular organisms to adjust cytoskeletal tension and adhesion strength 29 .

From a signaling perspective, we found that the extracellular and the Ras-GAP domains of Plexin-B2 are indispensable to rescue the $\mathrm{KO}$ phenotypes, in agreement with a high degree of 

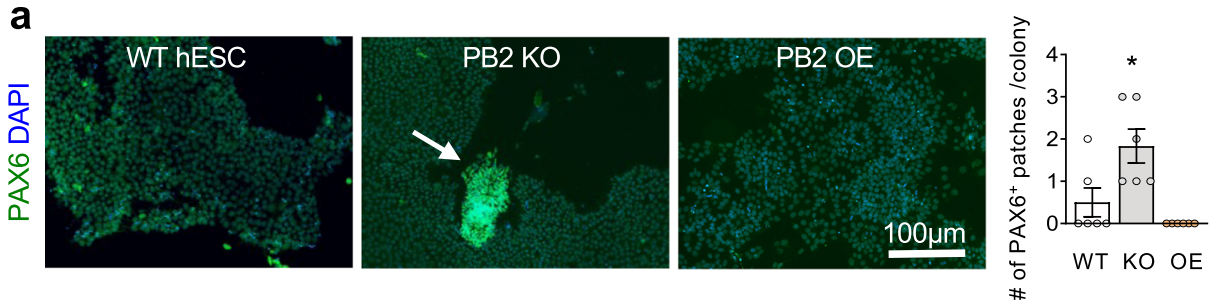

\section{b}

PB2 KO/WT hESC

C
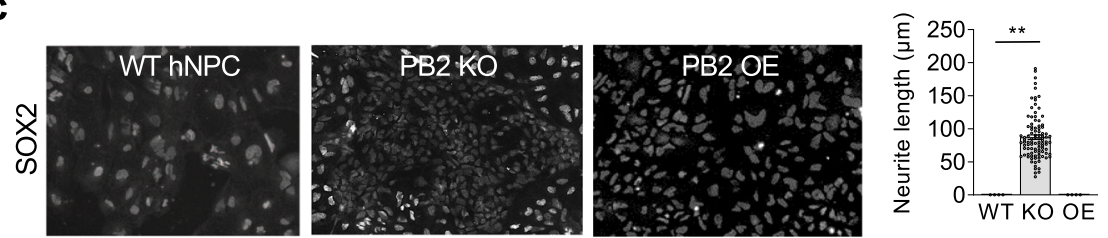

d
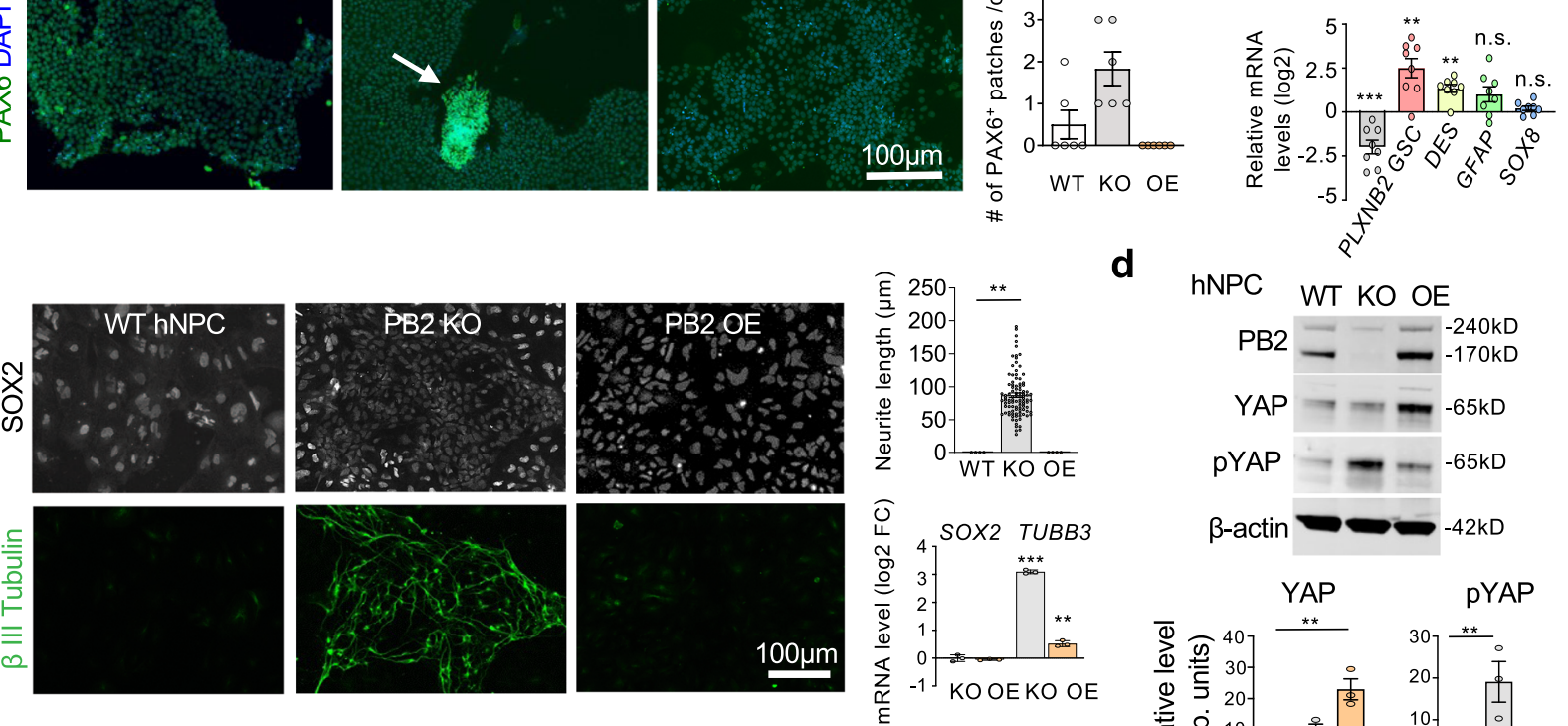

hNPC WT KO OE
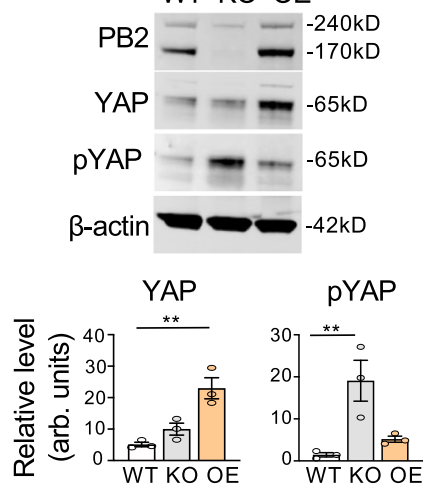

e

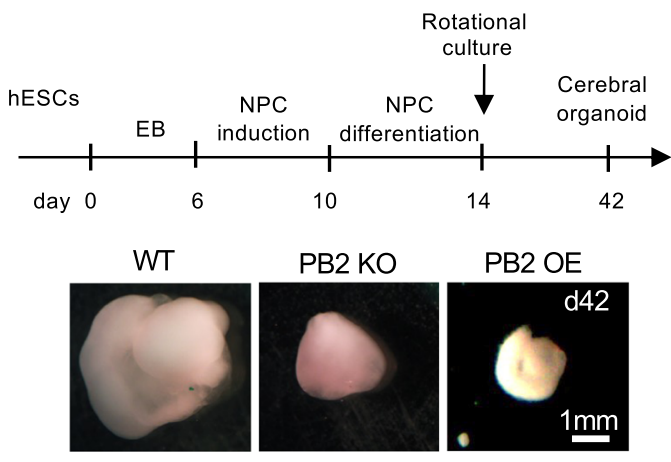

f
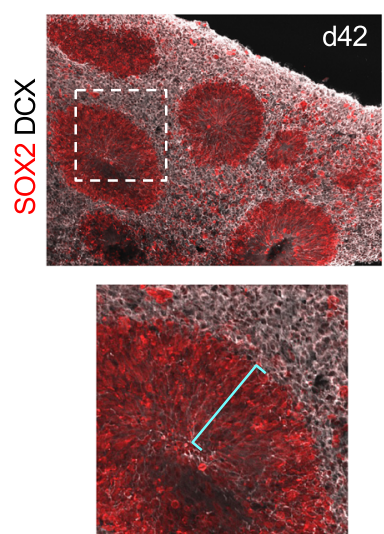

PB2 KO
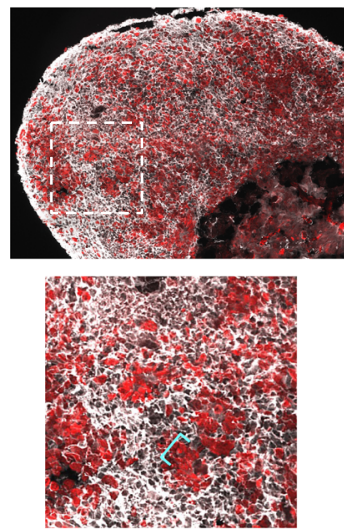

g

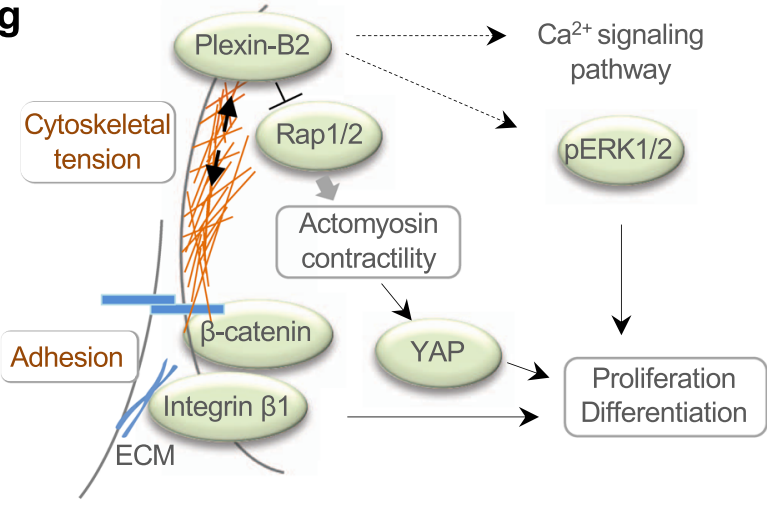

PB2 OE
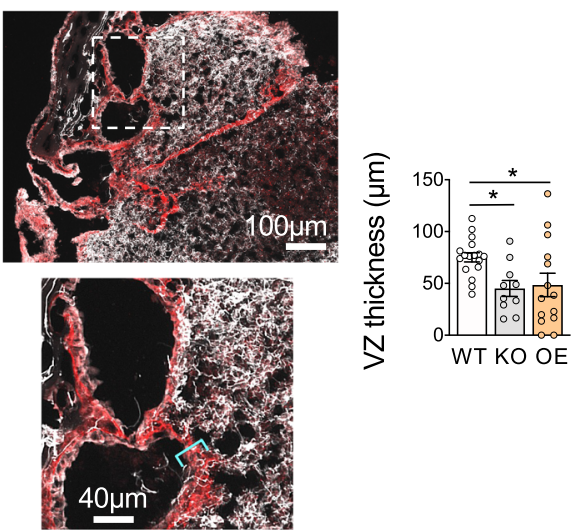

evolutionary conservation of these domains ${ }^{29}$. Plexin-B2 can be activated by class 4 semaphorins ${ }^{71}$; however, whether the mechanoregulatory function of Plexin-B2 requires semaphorins need further studies. In this context, it is notable that a semaphorin-independent mechanosensory function of Plexin-D1, a paralog of Plexin-B2, has recently been reported in endothelial cells, and this involves the bending of its extracellular domain in response to blood flow shear stress ${ }^{72}$. Whether this applies to
Plexin-B2 in regulating multicellular organization awaits future studies, but it is worth noting that the current study and the earlier study ${ }^{72}$ converge on the mechanical effects of Plexins in inducing cytoskeletal changes, calcium surge, and ERK1/2 activation.

The effects of Plexin-B2 on stem cell physiology may be linked to YAP and $\beta$-catenin signaling. We demonstrated that YAP can respond to Plexin-B2-mediated cytoskeletal tension, and can itself 
Fig. 8 Plexin-B2 affects stem cell differentiation and neuroepithelial architecture. a IF images and quantification show small patches of PAX6 ${ }^{+}$cells in Plexin-B2 deficient hESC colonies (arrow). One-way ANOVA followed by Tukey's post hoc test. $n=6$ colonies per group. ${ }^{\star} P=0.019$. Data represent mean \pm SEM. $\mathbf{b}$ qRT-PCR results confirm induction of marker genes for differentiation in Plexin-B2 deficient hESCs relative to WT. Note the verification of reduced PLXNB2 expression in KO cells. Two-sided unpaired $t$-test. $n=8$ samples per group. ${ }^{\star \star} P=0.0036$ (for GSC), ${ }^{\star \star} P=0.0015$ (DES), and ${ }^{\star \star \star} P=0.0002$ (PLXNB2). n.s. not significant. Data represent mean \pm SEM. $\mathbf{c}$ IF images show increased expression of $\beta$-III tubulin at the expense of SOX2 in PLXNB2 KO hNPCs derived from hESCs (clone KO\#2). Quantifications show enhanced neurite length (visualized by $\beta$-III tubulin IF) in PLXNB2 KO cells as compared to WT or PLXNB2 OE cells. One-way ANOVA followed by Tukey's multiple comparisons test versus WT. $n=4$ random fields per group. ${ }^{\star \star} P=0.0014$. Right, qRT-PCR results show relative transcript levels of SOX2 and TUBB3 in PLXNB2 KO and OE hNPCs relative to WT. One-way ANOVA followed by Tukey's multiple comparisons test versus WT. $n=3$ samples per group. ${ }^{\star \star} P=0.0048 ;{ }^{\star \star \star} P<0.0001$. Data represent mean \pm SEM. $\mathbf{d}$ WBs show levels of total YAP and PYAP in hNPCs in dependence of Plexin-B2. $\beta$-actin as a loading control. arb.units arbitrary units. One-way ANOVA followed by Dunnett's multiple comparisons test. $n=3$ samples per group. For $Y A P,{ }^{\star \star} P=0.0026$. For $P Y A P,{ }^{\star \star} P=0.0085$. Data represent mean $\pm \mathrm{SEM}$. e Timeline of cerebral organoid derivation from hESCs. Images show smaller size of cerebral organoids with Plexin-B2 perturbation relative to WT at matching time points (day 42). $\mathrm{f} \mathrm{IHC}$ images show SOX2+ neuroprogenitors and $\mathrm{DCX}^{+}$neuroblasts residing in ventricular structures in WT cerebral organoids, but in disarray in mutants. Magnified images are shown below, with brackets marking ventricular zone (VZ) thickness, as quantified in bar graphs on the right. One-way ANOVA followed by Tukey's multiple comparisons test versus WT. $n=17,10,14$ ventricular zones for $W T$, KO, and OE, respectively. ${ }^{\star} P=0.042$ (WT vs. KO) and ${ }^{\star} P=0.047$ (WT vs. OE). Data represent mean \pm SEM. $\mathbf{g}$ Model of the link of Plexin-B2 signaling with mechanoregulation and stem cell functions.

mediate cytoskeletal dynamics. Consistently, YAP mutation in fish models results in flattening of the body, indicating a requirement of YAP for tissue integrity ${ }^{47}$. Our study also established Rap1/2 as downstream effectors of Plexin-B2 in orchestrating actomyosin network, YAP, and ERK1/2 activation, as well as stem cell proliferation in hESC colonies. Intriguingly, Rap2A has been shown as a key intracellular signal transducer that relays $\mathrm{ECM}$ rigidity to YAP/TAZ ${ }^{7}$. This raises a tantalizing possibility that Rap1/2 may relay mechanical information from Plexin-B2 to YAP.

Interestingly, Worzfeld and colleagues have recently implicated Plexin-B1 and -B2 in mechanosensing during epidermal development to detect compressive forces, such as those elicited by cell confluency, resulting in YAP inactivation ${ }^{18}$. This study and ours converge on the roles of Plexins in enhancing the stiffness of cortical actin cytoskeleton, stabilizing cell-cell adhesive complexes, and conferring mechanosensitivity. However, perhaps reflecting tissue-specific roles, the two studies diverge on the way Plexin-B2 controls YAP activation and thereby cell proliferation and contact inhibition: while in hESCs, Plexin-B2 deletion resulted in decreased nuclear YAP and reduced proliferation, in epidermal stem cells, loss of Plexin-B1 and -B2 led to increased nuclear YAP and over-proliferation ${ }^{18}$. Two observations may explain these divergent functions: First, differentiation states of epidermis and hESCs are different; second, whereas epidermis responds to biomechanical constraints from compressive forces in confluent cell layers, human PSCs are insensitive to such contact inhibition ${ }^{64}$.

From a developmental point of view, our data solidify the principle that mechanical forces and tissue architecture provide an overarching signal to inform cell fate decisions ${ }^{73}$. Our data showed that Plexin-B2 deletion reduces cell-intrinsic stiffness of hESCs and hNPCs, and this is associated with neuronal cell fate commitment. This echoes a previous report that soft matrix substrate promotes neural differentiation of hESCs, while stiff substrate does not affect the pluripotency state ${ }^{74}$. Interestingly, in mesenchymal stem cells, the lineage specification is also sensitive to tissue elasticity, with softer matrix promoting neuronal while stiffer matrix promoting muscle differentiation ${ }^{75}$. Hence, cytoskeletal tension as controlled by Plexin-B2 exerts similar effects on cell fate commitment as substrate stiffness. Our study may thus point to strategies to accelerate neuronal differentiation by manipulating intrinsic cell mechanics.

The mechanoregulatory function of Plexin-B2 is more critical for structurally complex tissues such as ventricular zones and neuroepithelium. In cerebral organoids, Plexin-B2 perturbation caused ventricular malformation, reminiscent of the neural tube closure defect seen in Plxnb2 KO mice ${ }^{12}$, and echoing earlier reports that neural tube closure defect can arise from dysregulated mechanical interactions and tissue tension, thereby impacting cellular alignment and NPC function ${ }^{10}$. Our study also has implications for development and cancer. Indeed, reduced PlexinB2 activity has been suspected to contribute to the neurodevelopmental phenotypes of Phelan-McDermid syndrome $(22 \mathrm{q} 13$ deletion $)^{76}$. In breast cancer and glioblastoma, Plexin-B2 is upregulated and promotes tumor growth and invasion 77,78 . Resonating with our current study, we have also recently demonstrated that Plexin-B2 provides the biomechanical dynamics required for invasive growth of glioblastoma cells ${ }^{17}$.

In sum, our study identified a critical role of Plexin-B2 in organizing the actomyosin network and cytoskeletal tension during the multicellular organization of hESCs and hNPCs. Our data underscore the importance of force-mediated processes in controlling proliferation, cell fate specification, and tissue morphogenesis. Our findings may have broad implications for understanding neurodevelopmental disorders, tissue repair, and cancer biology.

\section{Methods}

hESC culture. All culture experiments were conducted using the WA09 (H9) $\mathrm{hESC}^{79}$ line obtained from the University of Wisconsin and validated at the Mount Sinai Stem Cell core facility. The Mount Sinai Stem Cell core conducts routine quality controls by verifying karyotype and mycoplasma-free status for all stem cell lines. Briefly, H9 hESC were seeded on matrigel-coated cell culture dishes $(1: 100$, Corning) and grown in mTeSR1 medium (STEMCELL Technologies). Cells were passaged around every 5 days by dissociation with Accutase (BD Biosciences) and addition of $2.5 \mu \mathrm{M}$ Rho-associated protein kinase (ROCK) inhibitor Thiazovivin (Millipore). The experiments were conducted at early hESC passages (P1 or P2) upon viral infections. The study was approved by the Embryonic Stem Cell Research Oversight Committee (ESCRO) at Icahn School of Medicine at Mount Sinai.

CRISPR genome editing for Plexin-B2 knockout. The CRISPR/Cas9-sgRNA sequence targeting PLXNB2 exon 3 was embedded inside a CRISPR/Cas9 lentivirus plasmid $^{80,81}$ to generate plentiCRISPRv2-sgPLXNB2 (target sequence: GTTCTCGGCGGCGACCGTCA). Lentiviral particles were produced in HEK293T cells (Takara, no. 632180) by co-transfection of lentiviral plasmid, envelope plasmid pMD2.G, and packaging plasmid psPAX2 (Addgene plasmids \#12259 and \#12260; deposited by Didier Trono, EPFL Lausanne). Low passage hESCs transduced with the lentivirus were selected with puromycin $(1 \mu \mathrm{g} / \mathrm{ml})$ for 7 days. Successful knockout of Plexin-B2 was confirmed by WB, demonstrating the absence of mature Plexin-B2 (170 kDa). A lentiviral vector (plentiCRISPRv2sgEGFP) expressing sgRNA targeting eGFP (target sequence: GGGCGAGGAGCTGTTCACCG) was used as control (WT)

Plasmids cloned for this study have been deposited at Addgene ("Roland Friedel Lab Plasmids"; https://www.addgene.org/Roland_Friedel/). 
CRISPR clones sequencing and karyotyping. To isolate clonal lines of hESCs that carry defined CRISPR mutations, after cells were transduced with lentiviruses (either targeting Plexin-B2 or EGFP as control) and selected with puromycin as described above, three colonies were picked, expanded, and sequenced for verifying mutations at the PLXNB2 locus. Three PB2 KO and its respective WT clones were confirmed. Normal karyotypes of selected clonal lines were confirmed by chromosome G-banding at Mount Sinai Genetic Testing Laboratory Sema4 (New York, USA).

\section{Lentiviral vectors for Plexin-B2 rescue, overexpression, and signaling} mutants. For rescue experiments, human PLXNB2 cDNA (entry clone HsCD00399262, DNA Resource Core at Harvard Medical School) was modified by site-directed mutagenesis to generate a CRISPR-resistant PLXNB2 cDNA, which was inserted into the lentiviral vector pLenti-PGK (plasmid backbone pLENTIPGK Neo DEST, Addgene \#19067; deposited by Eric Campeau \& Paul Kaufman) by Gateway LR Clonase II (Thermo Fischer Scientific). The resulting vector pLVPLXNB2 was then used to generate lentiviruses to transduce PLXNB2 KO hESCs for rescue/structural mutants' experiments. The pLV-PLXNB2 without CRISPRresistant mutation was used to generate lentivirus for overexpression experiments by transducing $\mathrm{H} 9$ hESCs.

The mutations in functional domains of Plexin-B2 were generated in the CRISPR-resistant PLXNB2 cDNA plasmid using the Phusion Site-Directed Mutagenesis Kit (Thermo Fischer): mGAP (mutation in the GAP domain: R1391A, R1392G), mRBD (mutation in the RBD domain: LSK 1558-1560 -> GGA), $\triangle$ VTDL (deletion of four last C-terminal amino acids VTDL of PDZ binding motif), and $\triangle \mathrm{ECTO}$ (lacking extracellular domain, deletion of amino acids 30 1189). The mutant cDNAs were transferred into pLenti-PGK destination vector by Gateway cloning as described above.

Doxycycline-inducible Plexin-B2 overexpression and shRNA KD. A plasmid for a lentiviral vector for doxycycline (Dox)-inducible human Plexin-B2 overexpression was generated by inserting human PLXNB2 cDNA by Gateway cloning into pLenti-CMVtight-Puro DEST (Addgene \#26430). Target cells were coinfected with a lentivirus expressing Tet-On $3 \mathrm{G}$ transactivator protein and hygromycin resistance (VectorBuilder, VB160122 -10094). Stable PLXNB2 Tet-On hESC lines were established by puromycin $(1 \mu \mathrm{g} / \mathrm{ml})$ and hygromycin $(200 \mu \mathrm{g} / \mathrm{ml})$ selection of coinfected cells.

Temporally controlled KD of Plexin-B2 at different phases of hESC colony expansion was achieved with TET-ON lentiviral vectors (Tet-pLKO-Puro backbone, Addgene \#21915, deposited by Dimitri Wiederschain) expressing Doxinducible shRNA targeting PLXNB2 and puromycin resistance marker (pLKO-TetOn-PLXNB2-shRNA1 and -shRNA2). A non-targeting shRNA vector (pLKO-TetOn-shRNA-Control) served as control (oligonucleotides used for construction of shRNA vectors are listed in file Supplementary Data 1). Stable PLXNB2 shRNA lines were established by puromycin selection $(1 \mu \mathrm{g} / \mathrm{ml})$ over 7 days. The PLXNB2 overexpression and the shRNA-mediated PLXNB2 KD were induced by adding $1 \mu \mathrm{g} / \mathrm{ml}$ Dox (MP Biomedicals) to the culture medium.

Doxycycline-inducible YAP overexpression and shRNA KD constructs. DNA plasmids for lentiviral vectors with doxycycline-inducible human YAP1 and YAP15SA cDNAs were generated by VectorBuilder, Inc.. Plasmids were designed using VectorBuilder website tools. In brief, human YAP1 plasmid was designed using an mRNA sequence from NCBI transcript NM_001282101, and human YAP1-5SA was designed based on ref. ${ }^{82}$ to generate an unphosphorylated CA form of YAP1. Both WT and mutant YAP1 plasmids are driven by a TRE3G promoter and have a CMV-puromycin-T2A-mCherry cassette inserted after the gene of interest to facilitate the selection of transfected cells. Target cells were coinfected with a lentivirus expressing Tet-On 3G transactivator protein and hygromycin resistance (VectorBuilder, VB160122 -10094).

YAP short hairpin (sh)RNA lentiviral expression vectors were cloned by ligating oligonucleotides encoding the shRNA hairpins (Integrated DNA Technologies) into the pLKO-Tet-On vector (Addgene \#21915) to have temporal control of YAP1 KD (oligonucleotide sequences are listed in file Supplementary Data 1). All plasmids were validated using restriction enzymes and Sanger sequencing (Macrogen) using the primer pLKO-shRNAseq (ggcagggatattcaccattatcgtttcaga).

Lentiviral constructs for RAP1/2 small GTPases. To validate the relevance of the Ras family of small GTPases, human RAP2A cDNA was modified to generate a CA (RAP2A-V12) and DN (RAP2A-N17) isoforms using site-directed mutagenesis (Thermo Fischer). The RAP2A isoforms were then inserted into the lentiviral vector pLenti-PGK Neo or Puro DEST by Gateway LR Clonase II (Thermo Fischer). For RAP1B, CA (bovine Flag-RAP1B-V12, Addgene \#118323) and DN (bovine Flag-Rap1B-N17, Addgene \#118322) were inserted into the same lentiviral vectors as described above for RAP2A. The resulting vectors were used to transduce WT, PB2 KO, and PB2 OE hESCs.

hESC colony and 3D aggregation assays. For adherent hESCs culture, cells were plated at a density of $2.5 \times 10^{4}$ in Matrigel-coated six-well plates. Individual colonies were imaged from day 1 to day 6 with a CKX53 inverted microscope (Olympus) using the Olympus cellSens Entry software, and colony sizes were measured using ImageJ.

A 3D aggregation assay was performed by culturing $4 \times 10^{4} \mathrm{hESC}$ resuspended in mTeSR1 medium in low-adhesion 24-well plates with the addition of $10 \mu \mathrm{M}$ Thiazovivin. The size of hESC aggregates was evaluated after $48 \mathrm{~h}$. For the hanging drop assay, $2.5 \times 10^{4} \mathrm{hESCs}$ were resuspended in $10 \mu \mathrm{l}$ of mTeSR1 medium with $10 \mu \mathrm{M}$ Thiazovivin for $24 \mathrm{~h}$. Images were taken using a Nikon SMZ745D microscope, and areas of clustered cells were measured using ImageJ.

Culture of hESCs on hydrogels with different stiffness. To investigate the effects of substrate stiffness on YAP localization in hESCs carrying Plexin-B2 mutations, we used synthetic peptide hydrogels (Manchester Biogel) of different stiffness: PeptiGel-alpha $4(\sim 1.0 \mathrm{kPa})$ and PeptiGel-gamma $2(\sim 5.0 \mathrm{kPa})$. Gel solution was centrifuged to remove air bubbles and $100 \mu \mathrm{l}$ each was distributed into chambers of a four-chamber $35 \mathrm{~mm}$ glass-bottom dish (CellVis). The chambers containing hydrogel solutions were overlayed with DMEM/F12 media containing Matrigel (1:100, Corning) and incubated for $1 \mathrm{~h}$ at $37^{\circ} \mathrm{C}$ to induce gelling. Media was carefully removed and hESCs were seeded on the top of the gels at a density of $10^{4}$ cells per chamber in mTeSR1 media containing $2.5 \mu \mathrm{M}$ Thiazovivin (Millipore). As control condition, hESCs were plated into Matrigel-coated glass coverslips only. The media was replaced daily and the cells were maintained for 3 days before fixation and imaging.

Calcium imaging of hESCs. For calcium imaging of hESCs, $4 \times 10^{4}$ cells were seeded in a Matrigel-coated four-chamber $35 \mathrm{~mm}$ glass-bottom dish (CellVis) and grown for 3 days. The cells were loaded in mTeSR1 medium supplemented with $10 \mu \mathrm{M}$ Fluo-3 AM (Biotium; diluted in $20 \%$ Pluronic F-127) for $30 \mathrm{~min}$ at $37^{\circ} \mathrm{C}$, washed, and superfused with fresh mTeSR1 medium. Fluo-3 AM was excited at $488 \mathrm{~nm}$ and emitted fluorescence above $505 \mathrm{~nm}$ was recorded by an LSM 780 confocal microscope (Zeiss) in frame scan mode on a heated stage. Hoechst 33342 (1:2,000, Thermo) and CellMask deep red (1:2,000, Thermo) dyes were used for nuclear and plasma membrane staining, respectively.

Neural differentiation. Human neural progenitor cells (hNPCs) were generated by a single-cell monolayer protocol using STEMDiff Neural Induction Medium (StemCell Technologies). For the monolayer protocol, hESCs were plated at a density of $2.5 \times 10^{5} \mathrm{cells} / \mathrm{cm}^{2}$ in STEMDiff medium, and on day 6 the cells were replated at the high confluence. After day 11 the cells were plated on glass coverslips coated with Matrigel and analyzed by immunocytochemistry (ICC) to confirm the NPCs identity and used for subsequent experiments. Neurite length was measured using the ImageJ plugin Simple Neurite Tracer.

Cerebral organoid derivation from hESCs. Cerebral organoids were generated from hESCs by forming the first embryoid bodies that were subsequently transferred to Matrigel droplets for maturation into organoids ${ }^{66,67}$. Briefly, H9 hESCs were dissociated into single cells with Accutase (BD Biosciences) and plated in ultra-low-attachment 96-U-well plates (Costar) at a density of 9000 cells/well $(150 \mu \mathrm{l})$ in mTeSR1 medium (STEMCELL Technologies) containing $10 \mu \mathrm{M}$ Thiazovivin (Millipore). After 6 days, embryoid bodies were cultured in STEMDiff Neural Induction Medium (STEMCELL Technologies). The neuroepithelial tissue that formed over the next 4-6 days was transferred into $30 \mu$ Matrigel (Corning) droplets and grown for 4 days in stationary culture in a medium composed of DMEM-F12 (Invitrogen), Neurobasal medium (Invitrogen), N2 supplement (Invitrogen), human insulin (Sigma), GlutaMAX supplement (Gibco), MEMNEAA (Gibco), penicillin-streptomycin (Sigma), 2-mercaptoethanol (Millipore), and B27 supplement minus vitamin A (Invitrogen). This was then followed by culturing the droplets in six-well plates on an orbital shaker with the addition of a B27 supplement plus vitamin A (Invitrogen) to the media to promote neuronal differentiation. At day 42 cerebral organoids developed to a size of $\sim 3-5 \mathrm{~mm}$ in diameter and were analyzed.

Immunocytochemistry and immunofluorescence. For ICC, cells were fixed in 4\% formaldehyde in PBS at room temperature for $10 \mathrm{~min}$, washed $3 \mathrm{x}$ with PBS, permeabilized and blocked with 5\% donkey serum, and $0.3 \%$ Triton X-100 in PBS for $1 \mathrm{~h}$. Primary antibodies were incubated at $4{ }^{\circ} \mathrm{C}$ overnight in dilution buffer composed of PBS with $1 \%$ BSA and $0.3 \%$ Triton X-100. Secondary antibodies were incubated in dilution buffer at room temperature for $1 \mathrm{~h}$ together with nuclear staining (DAPI, 1:1000; Thermo Fisher). For most preparations, the cells were also counterstained for F-actin with Alexa Fluor 568 Phalloidin (Thermo Fisher).

For histological analysis of cerebral organoids by IF, cerebral organoids were fixed in $4 \%$ paraformaldehyde/PBS at $4{ }^{\circ} \mathrm{C}$ for 15 min, washed with PBS, cryoprotected in $30 \%$ sucrose at $4^{\circ} \mathrm{C}$ overnight, embedded in OCT (Fisher HealthCare), and frozen on dry ice. The organoids were sectioned using a cryostat (Leica) at $12 \mu \mathrm{m}$ thickness and the cryosections were used for antibody staining following the protocol described above for ICC. 
Western blot. For WB, freshly collected samples were lysed in RIPA buffer (Sigma) containing protease (Fisher Scientific) and phosphatase inhibitors (Sigma). Protein concentrations were determined using a BCA assay (Thermo Scientific). Proteins were resolved on $4-12 \%$ polyacrylamide NuPAGE gels (Invitrogen) using the XCell SureLock system (Invitrogen) and transferred to nitrocellulose membranes (Li-Cor Biosciences). The immunoreactive bands were detected by fluorescent ODYSSEY infrared imaging system (Li-Cor Biosciences). Equal protein loading was controlled by probing the blots with an antibody against a housekeeping control protein. Uncropped WBs can be found in the Source data file.

Antibodies. The following primary antibodies were used for ICC/IF: anti-sheep plexin-b2 (ecd) (1:300, R\&D systems, AF5329), anti-rabbit plexin-b2 (icd) (1:200, Abcam, ab193355), anti-rabbit sox2 (1:200, Abcam, ab97959), anti-guinea pig dcx (1:500, EMD Millipore, ab2253), anti-rabbit pmlc2 (1:200, Cell Signaling, 3671), anti-rat e-cadherin (1:200, Life Technologies, 131900), anti-mouse n-cadherin (1:200, BD Bioscience, 610920), anti-rabbit zo-1 (1:200, Thermo, 61-7300), antimouse integrin $\beta 1$ (active, huts-4) (1:200, EMD Millipore, MAB2079Z), antimouse, oct4 (1:500, Abcam, ab184665), anti-rabbit pFak (1:200, Thermo, 44$624 \mathrm{G})$, anti-rabbit nanog (1:200, Abcam, ab109250), anti-rabbit caspase 3 (1:500, R\&D systems, AF835), anti-mouse yap (1:200, Santa Cruz, sc-101199), anti-mouse $\beta$-catenin (1:200, BD Bioscience, 610153), anti-mouse pax6 (1:200, Abcam, ab78545), anti- $\beta$ III tubulin (tuj1) (1:100, R\&D systems, MAB1195), anti-mouse paxillin (1:200, Invitrogen, AHO0492), anti-mouse p-vimentin (phospho S55) (1:200, Abcam, ab22651), anti-rabbit FN (1:200, EMD Millipore, ab2033).

The following secondary antibodies were used for ICC/IF: Alexa Fluor 488, 594, or 647-conjugated donkey anti-goat, -rabbit, -rat, or -mouse IgG, and anti-guinea pig IgG (Jackson ImmunoResearch Laboratories, 1:300).

For function-blocking assay: anti-mouse integrin $\beta 1$ (P5D2) $(10 \mu \mathrm{g} / \mathrm{ml}$, Santa Cruz, sc-13590) and ChromPure Mouse IgG, whole molecule (1:1,000; Jackson ImmunoResearch Laboratories, 015-000-003).

The following primary antibodies were used for WB: anti-rabbit $\beta$-actin (1:10,000, Sigma, A1978), anti-sheep plexin-b2 (ecd) (1:500, R\&D Systems, AF5329), anti-rabbit $\beta 1$-integrin (4706 s) (1:1000, Cell Signaling, 4706), anti-mouse yap (1:500, Santa Cruz, sc-101199), anti-rabbit phospho-yap (Ser127) (1:1000, Cell Signaling, 4911 S), anti-rabbit phospho-p44/42 mapk (erk1/2) (thr202/tyr204) (1:1000, Cell Signaling, $9101 \mathrm{~S})$, anti-mouse p44/42 mapk (erk1/2) (3a7) (1:1000, Cell Signaling, 9107 S), anti-mouse $\beta$-catenin (1:1000, BD Bioscience, 610153), anti-rabbit sox2 (1:10,000, Abcam, ab97959), anti-rabbit pax6 (1:2000, BioLegend, 901302), anti-rabbit gapdh (1:1000, Cell Signaling, 2118), anti-rabbit h3k4me2 $(0.5 \mu \mathrm{g} / \mathrm{ml}$, Active Motif, 39914$)$. The following secondary antibodies were used: IRDye 800CW donkey anti-mouse (1:10,000, Li-Cor Biosciences, 926-32212), 680RD-donkey anti-goat (1:10,000, Li-Cor Biosciences, 926-68024), and anti-rabbit (1:10,000, Li-Cor Biosciences, 926-68073).

Staining of cells for cytoskeleton and membrane components. To visualize F-actin in fixed cells, staining with fluorescently labeled phalloidin was performed (Alexa Fluor 568 phalloidin (1:100, Invitrogen, A12380), Alexa Fluor 647 phalloidin (1:500, Invitrogen, A22287). F-actin and pMLC2 foci were measured with the assistance of the ImageJ plugin BioVoxxel Toolbox Cluster Indicator. $\beta$-catenin peaks were measured using the ImageJ plugin BAR Finding Peaks.

To observe F-actin in live cells with the LifeAct-mScarlet sensor protein, hESCs and hNPCs were infected with lentiviral preparations expressing LifeActmScarlet ${ }^{44}$. The cells were selected with G418 $(200 \mu \mathrm{g} / \mathrm{ml})$ over 7 days and expanded for subsequent experiments. For staining with the fluorescent SPY555Actin probe (Cytoskeleton, Inc), live hESCs were incubated with the dye for $1 \mathrm{~h}$ and imaged using the LSM 780 confocal microscope on a heated stage at $37^{\circ} \mathrm{C}$ and $5 \% \mathrm{CO}_{2}$ for probing the actin dynamics.

For CellMask (Thermo) plasma membrane staining, live hESCs were incubated with dye for 5-10 min and imaged immediately to record the dynamic changes of plasma membranes and cell borders. Alternatively, cell membranes were visualized in live cells with MemGlow 488 dye (1:200, Cytoskeleton, MG-01). Cell nuclei were stained with Hoechst 33342 dye (Thermo) or NucSpot Live 650 (Biotium). Based on the live staining described above, the circularity index of cells was calculated with the formula: $4 \pi \mathrm{x}$ area/perimeter ${ }^{\wedge} 2$. A value of 1.0 indicates a perfect circle; a value approaching 0.0 indicates an increasingly elongated shape.

Total internal reflection microscopy (TIRF) and focal adhesion segmentation. TIRF microscopy was performed using a Leica DMi8 Infinity TIRF microscope and LASX (v3.7.0.20979). Focal adhesion morphometrics were assessed using IF staining of paxillin (at an imaging depth of $90 \mathrm{~nm}$ ), which was imaged under PBS supplemented with ProLong Live antifade agent (Thermo P36975) Nuclear and actin channels were obtained simultaneously by epifluorescence using IF staining of phalloidin and DAPI as mentioned above. Images were captured using a 1.4 NA Leica $63 \mathrm{X}$ oil TIRF objective at room temperature.

For quantification of focal adhesion morphometrics, binary masks of the FAs were generated in Fiji ${ }^{83}$. First, the background of the paxillin image had its background subtracted using the rolling ball method with $r=30$. Next, the image was auto-thresholded using the moments method on a dark background. This was then converted to a binary mask and the "Fill Holes" operation was applied.
Following this, the actin channel was used to generate a cell mask in the following manner: the actin channel was auto-thresholded using the mean method with a dark background. This was then converted to a binary mask with the "Dilate", "Fill Holes", and "Open" operations performed on it. The final mask used for quantification was the result of applying the logical AND operation on both masks. Segmented images of the nuclei were obtained using Cellpose on the DAPI channel. After this, the binary images were analyzed using an in-house Matlab script. To calculate the number of FAs per cell, the number of segmented FAs was divided by the number of segmented nuclei for each region imaged.

High-content image analysis of cell morphologies. hESCs were seeded on a four-chamber $35 \mathrm{~mm}$ glass-bottom dish (CellVis) at a density of $2 \times 10^{4}$ cells/well and cultured at $37^{\circ} \mathrm{C}$ for $3-5$ days. hNPCs were seeded at $4 \times 10^{4}$ cells on glass coverslips $25 \mathrm{~mm}, \# 1 \frac{1 / 2}{}$ (Electron Microscopy Sciences) and cultured at $37^{\circ} \mathrm{C}$ for 2 days. The cells were fixed in $4 \%$ formaldehyde in PBS at room temperature and ICC was carried out as described in the respective section above. Cells were then incubated with primary antibodies in dilution buffer at $4{ }^{\circ} \mathrm{C}$ overnight and incubated with secondary antibodies (Alexa Fluor 488 and 647; Jackson ImmunoResearch) at 1:300 dilution in dilution buffer for $1 \mathrm{~h}$ at room temperature. Subsequently, the cells were counterstained for F-actin and nucleus with Phalloidin 568 (Thermo Fisher) and DAPI, respectively. Image acquisition (30 fields per condition) was carried out on LSM 710 confocal microscope (Zeiss) using 40X objective and zoom 0.6 .

Cell segmentation was performed using Cellpose ${ }^{84}$. Briefly, nuclear objects were identified using the DAPI channel, and the corresponding cell objects were identified using the innate propagation algorithm of Cellpose coupled with the contrast-enhanced phalloidin or $\beta$-Catenin channel to define cell boundaries. Image analysis and quantification were performed using Matlab where multiparametric analysis was performed for the four channel images, including morphometric (size and shape) and intensity. Measurements were exported as xls files and GraphPad Prism was used to generate box plots.

To calculate cell-cell distances from confocal microscopy images, centroid distances were calculated from DAPI images. We calculated the distance between every nucleus in each image. The five nearest cells for each cell in the image were picked up for histogram plotting. The histogram was normalized using a Probability density estimate function. All centroid distance calculations and histogram plotting were performed with Matlab R2018a software (MathWorks).

Time-lapse video microscopy. For SPY555-Actin time-lapse microscopy, hESCs were plated at a density of $2 \times 10^{4}$ cells per well in a four-chamber $35 \mathrm{~mm}$ glassbottom dish (Cellvis) and left growing for $18 \mathrm{~h}$. Live-cell time-lapse video microscopy was carried out for $21 \mathrm{~h}$ with frames taken every $20 \mathrm{~min}$, using LSM 780 confocal microscope (Zeiss), with environmental control chamber conditions set at $37^{\circ} \mathrm{C}$ temperature and $5 \% \mathrm{CO}_{2}$. The analysis was carried out using Imaris software (Oxford Instruments).

EdU Click-iT assay. To assess the proliferative potential of hESCs, cells were plated at a density of $2 \times 10^{4}$ in Matrigel-coated eight-chamber slides, and after 3 days of culture, $10 \mu \mathrm{M}$ of the nucleotide analog EdU (Life Technologies) was added to the medium for $30 \mathrm{~min}$. The cells were immediately fixed in $4 \%$ formaldehyde at room temperature for $10 \mathrm{~min}$ and washed $3 \mathrm{x}$ with PBS. EdU Click-iT assay was performed accordingly to manufacturer's instruction (Thermo) followed by DAPI staining. The number of EdU ${ }^{+}$cells was quantified using Photoshop CS5 (Adobe) and ImageJ.

EdU Click-iT flow-cytometry assay. hESC and hNPCs were grown as 2D cultures on Matrigel-coated dishes and treated with $10 \mu \mathrm{M}$ EdU (Life Technologies) for 20 and $90 \mathrm{~min}$, respectively. The cells were harvested with Accutase, washed with PBS, pelleted, and fixed in Click-iT fixative at room temperature for $15 \mathrm{~min}$, protected from light. The cells were washed with 1\% BSA in PBS, pelleted, and resuspended in $1 \mathrm{X}$ Click-iT saponin-based permeabilization and wash reagent and click labeled with EdU Click-IT flow-cytometry assay, accordingly manufacturer's instruction (Thermo). DAPI (Invitrogen) was added to cell suspensions at a concentration of $5 \mu \mathrm{g} / \mathrm{ml}$ to stain the nuclei. Cells were resuspended in FACS buffer (Hibernate-E low fluorescence, BrainBits) with $0.2 \%$ BSA and $20 \mu \mathrm{g} / \mathrm{ml}$ DNase (Worthington) and passed through a 70- $\mu \mathrm{m}$ mesh filter. Cell suspensions were analyzed by flow cytometry (BD LSRII) and cell cycle data was evaluated with FACSDiva (BD) and FlowJo software (FlowJo LLC).

Subcellular fractionation. A subcellular protein fractionation kit (Cell Signaling) was used to separate cell lysates into cytoplasmic (Cyto), membrane (Mem), and nuclear (Nuc) fractions based on the use of detergents. All procedures were performed accordingly to the manufacturer's protocol. The fractions were mixed with 3x SDS loading buffer plus DTT and analyzed by WB.

RNA extraction, library preparation, and RNA-seq. Total RNA from WT, PB2 $\mathrm{KO}$, and PB2 OE hESCs were isolated using an RNeasy Micro kit (Qiagen), following the recommended instructions for on-column DNase digestion. RNA 
quality and concentrations were measured using an Agilent Bioanalyzer (Agilent RNA 6000 Pico), and samples with RNA integrity number scores higher than 7 were used for the experiments. Poly(A) mRNA were isolated using NEBNext Poly(A) mRNA Magnetic Isolation Module (NEB E7490S) and cDNA libraries for Illumina next-generation sequencing were prepared with NEBNext Ultra Directional RNA Library Prep Kit (NEB E7760S). Three independent samples for each condition were barcoded using NEBNext Index primers (NEB, E7335S), pooled together as one sample, and ran on an Illumina platform HiSeq2500 with one-sided 75 base reads, with about 30 million reads per sample.

Differential gene expression analysis. Raw RNA-seq reads were mapped to the hg38 genome using HISAT2 ${ }^{85}$. Counts of reads mapping to genes were obtained using featureCounts software of Subread package against Ensembl v90 annotation $^{86}$. Differential expression analysis was carried out using the DESeq2 package ${ }^{87}$, and PCA in the DESeq2 package was utilized to identify outliers. Unsupervised clustering of samples and of DEGs was performed by calculating for each gene the transcripts-per-million (TPM) value, filtering for genes with a mean expression value of $>20$ TPM, and selecting genes with a differential expression of at least twofold between any of the samples. The hierarchical clustering analysis was performed with the clustergram function using Matlab R2018a (MathWorks).

Gene ontology analysis of common and exclusive PB2 KO and PB2 OE DEGs was performed with the ENRICHR resource (http://amp.pharm.mssm.edu/Enrichr/; accessed $05 / 2020)$. The scales are ranked by "Combined Score" $(\log p$ value multiplied by the $z$-score of the deviation from the expected rank). The same gene lists were also analyzed by NetworkAnalyst 3.0 (https://www.networkanalyst.ca/) and used to visualize Reactome-based gene categories ${ }^{88}$. The most relevant pathways were selected to display interactive analytics of gene networks. Ingenuity pathway analysis (IPA) was performed to compare PB2 KO with PB2 OE DEG lists (https://www.qiagenbioinformatics.com/products/ingenuity-pathway-analysis/; accessed 07/2020)

QRT-PCR array and validation. Cells were lysed using RLT buffer (Qiagen) plus 2-mercaptoethanol (Millipore), and lysates were stored at $-80^{\circ} \mathrm{C}$ until RNA extraction. Total RNA was isolated using the RNeasy Mini Kit (Qiagen). RNA was converted to cDNA using oligo $(\mathrm{dT})$ primer, and quantitative PCR reactions were set up using the PerfeCTa SYBR Green FastMix ROX qPCR kit (QuantaBio). Quantitative RT-PCR array analysis was performed using a hESC-specific PCR array (PAHS-081Y; SABiosciences). Reactions were run on an ABI/Life Technologies 7900HT real-time PCR instrument. The statistical analysis and plotting were performed on the RT2 Profiler PCR Array Data Analysis Webportal (http://dataanalysis.qiagen.com/pcr/arrayanalysis.php).

Validation of qRT-PCR array and for PLXNB2 and YAP1 and its target genes (CYR61, CTGF, and ANKRD1) were performed with individual qRT-PCR reactions (primers sequences are listed in file Supplementary Data 1). Relative mRNA expression levels were determined by $\triangle \triangle \mathrm{Ct}$ method relative to GAPDH or ACTB housekeeping gene controls. qRT-PCR data are average from at least three replicates for each sample.

\section{Treatment with YAP inhibitor VP or $\boldsymbol{\beta}$-catenin inhibitor cardamonin. hESCs} were seeded at a density of $10^{4}$ cells in Matrigel-coated eight-chamber slides, and after 3 days of culture, $0.5 \mu \mathrm{M}$ of YAP inhibitor VP (Sigma) was added to the media for further culture of $24 \mathrm{~h}$. The $\beta$-catenin inhibitor cardamonin $(2 \mu \mathrm{M}$; Tocris $)$ was added after 2 days of culture for further culture of $48 \mathrm{~h}$. Cells were fixed in $4 \%$ formaldehyde/PBS at room temperature for $10 \mathrm{~min}$ and washed $3 \mathrm{x}$ with PBS before immunostaining and imaging.

For hNPC induction, hESCs were seeded according to the neural differentiation protocol described above and the $\beta$-catenin inhibitor cardamonin $(0.5 \mu \mathrm{M}$; Tocris) was added in the fresh media daily from day 1 to day 10. The YAP inhibitor VP $(0.5 \mu \mathrm{M}$; Sigma) was added in two $24 \mathrm{~h}$ pulses (days 5 and 9 ) to reduce toxicity effects.

Stimulating hESCs with Sema4C. For Sema4C coating of culture dishes, Sema4C$\mathrm{Fc}$ (R\&D Systems) at 2,10 , and $50 \mu \mathrm{g} / \mathrm{ml}$ was mixed with Matrigel, and dishes were incubated for $1 \mathrm{~h}$ at $37^{\circ} \mathrm{C}$. hESCs were seeded at a density of $4 \times 10^{4}$ cells per well and cultured for 2 days. The colonies were fixed, stained for cytoskeleton markers, and imaged.

For treatment with Sema4C soluble in media, hESCs were seeded at a density of $10^{4}$ cells per well of the eight-chamber slide and cultured for 2 days before being treated by addition of $2 \mu \mathrm{g} / \mathrm{ml} \mathrm{Sema} 4 \mathrm{C}-\mathrm{Fc}$ (R\&D Systems) to culture media for further 2 days.

Cytoskeletal drugs and integrin antibody blocking experiments. Cytoskeletal drugs and integrin antibody blocking experiments were carried out directly in live hESCs transfected with LifeAct_mScarlet or SPY555-Actin for Y-16 treatment. Drugs or vehicles were added to media, and cells were incubated for $3 \mathrm{~h}$ in a cell incubator. Drugs were used at the following concentrations: Rock Inhibitor Y-27632 $(20 \mu \mathrm{M})$, blebbistatin $(10 \mu \mathrm{M})$, Rho-GEF inhibitor Y16 $(25 \mu \mathrm{M})$, and latrunculin $(5 \mu \mathrm{M})$. Integrin $\beta 1$ antibody (P5D2; sc-13590) $10 \mu \mathrm{g} / \mathrm{mL}$ or IgG antibody (used as control) were added to cells in suspension for $15 \mathrm{~min}$ before plating cells in Matrigel-coated coverslips. The mTeSR media was supplemented with $2.5 \mu \mathrm{M}$ Thiazovivin (Millipore) and cells were incubated for $1 \mathrm{~h}$ before imaging. After incubation hESCs were treated with SPY555-Actin and CellMask dye (Thermo) and imaged immediately using LSM 710 confocal microscope (Zeiss).

AFM elastography. AFM measurements were conducted using an Asylum MFP 3D-BIO AFM equipped with environmental thermal and vibrational control coupled with an Olympus IX-80 inverted spinning disk confocal microscope ${ }^{43}$. For the experiments, hESCs were plated on $60 \mathrm{~mm}$ Matrigel-coated plastic dishes at a density of $5 \times 10^{5}$ for WT and PLXNB2 OE groups and $7.5 \times 10^{5}$ cells for the PLXNB2 KO group (to compensate for slower colony expansion). hNPCs were plated at a density of $4.2 \times 10^{6}$ for WT and PLXNB2 OE groups and $6.3 \times 10^{6}$ cells for PLXNB2 KO group on $60 \mathrm{~mm}$ dishes. After 3 days in culture, the plates were secured to the AFM and maintained at $37^{\circ} \mathrm{C}$ for the entire duration of the AFM experiment. A gold-coated silicon nitride probe with a triangular-shaped body, blunted pyramidal tip, and nominal spring constant of $0.1 \mathrm{~N} / \mathrm{m}$ (Cat \#: TR400PB Asylum) was used for cell measurements and calibrated using the thermal noise method. The Hertzian model was used to fit our indentation data for its elastic modulus, where this model called for probe tip half-angle $\left(9^{\circ}\right)$, probe material (silicon nitride), and sample Poisson's ratio $(v=0.45)$. To measure the colony elastic modulus a $24 \times 24$ indentation array was conducted over a $64 \mu \mathrm{m}^{2}$ region of the colony (avoiding colony edges). The AFM was set to conduct its indentation array at an indentation rate of $0.5 \mathrm{~Hz}$ and indent each time until the probe deflected $50 \mathrm{~nm}$ (about $6 \mathrm{nN}$ of force), which would trigger its retraction. For hESCs, each condition was measured for six-seven different colonies across different dishes from independent preparations. For hNPCs, each condition was measured for three areas across different dishes. For each cell group, every indentation was aggregated into a pool of measurements which was then averaged to return a mean colony stiffness. This aggregation allowed for proper sampling power regarding biological variability and batch variation. Due to the phenotypic nature of hNPCs, NPC cultures had open/dish exposed areas, which translated into direct dish measurements during AFM indentations, a phenomenon called substrate effect. To avoid these unwanted influences, NPC modulus values larger than $20 \mathrm{kPa}$ were filtered out (expected NPC stiffness $\sim 5 \mathrm{kPa}$ ). Mean colony stiffness trends and statistical comparisons were done on both filtered and unfiltered NPC data and confirmed to be equivalent.

Cell topology imaging via AFM. In addition to elasticity measurements, the atomic force microscope (AFM) can image colony topology via direct contact. We employed the AFM imagining technique called contact mode imaging whereby a feedback loop is set upon the probe as it is told to slide across, and track, the surface of the colony in a raster scanning manner. hESCs were prepared as above except that cells were fixed with formaldehyde for $15 \mathrm{~min}$. This allowed preservation of colony structure and features throughout the duration of AFM imaging, as well as increase colony robustness during extended AFM probe interaction. This step was acceptable for contact mode imaging but would not be appropriate for elasticity measurements as fixing may change the cells' elastic properties. The same type of probe used for AFM elasticity measurements was selected for contact mode imaging (see methods above), its $0.1 \mathrm{~N} / \mathrm{m}$ spring constant and $42 \mathrm{~nm}$ tip radius allowed for gentler contact imaging and detection of finer cell features. A $90 \mu \mathrm{m}^{2}$ region central to the cell colony was selected for imaging. The probe was prescribed to move at a speed of $200 \mu \mathrm{m} / \mathrm{second}$ and scan its $90 \mu \mathrm{m}^{2}$ region in 256 segments, these 256 segments were then stitched together for a complete topography map. Scans used a setpoint $=0.8 \mathrm{~V}$. Topology images revealed ruffles across the cell membrane particularly for the PLXNB2 KO group. To quantify these ruffles a profile was taken through a selected cell, perpendicular to the ruffle direction. That profile was then counted for cell membrane features (peaks) of amplitude greater than $15 \mathrm{~nm}$ of probe deflection. Each cell was measured for four profiles, resulting in a total (sum) of ruffles per cell. Five cells were selected per image (two images per cell type) and then averaged for a mean \# of ruffles per cell.

FRET analysis of RAP1/2 activation status and vinculin tension biosensor. For FRET analysis of RAP1/2 activation status, hESCs were transfected with RAP1A and 2 A FRET-based biosensor probes derived from original plasmids obtained from Dr. Hahn and Dr. O'Shaughnessy, University of North Carolina (UNC) Chapel Hill ${ }^{89}$.

For the vinculin tension biosensor, human NPCs were transfected with vinculin tension biosensor using lentiviral particles as described above. Forty-eight hours after transduction, cells were sorted by FACS (BD FACSAria IIu). Media was then supplemented with CloneR (Stem-Cell Technologies) and cells were expanded for the imaging experiment.

Analysis of FRET was performed by live-cell FRET imaging using Leica SP5 DMI confocal microscope equipped with temperature and $\mathrm{CO}_{2}$ control ${ }^{45}$ at the Mount Sinai microscopy core. eCFP was excited with a $458 \mathrm{~nm}$ emission line using an Argon ion laser and eYFP was excited with a $515 \mathrm{~nm}$ emission line. eCFP, FRET, and eYFP channels were simultaneously imaged using an internal GaAsp detector collecting the emission from a range of wavelengths appropriate for each channel: For eCFP channel: $463-520 \mathrm{~nm}$, FRET channel: $520-620 \mathrm{~nm}$, and eYFP channel: 520-620 nm. Intensity-based ratiometric FRET indices and heatmaps were 
obtained on a per-cell (for RAP1/2) and per-focal adhesion (vinculin tension biosensor) basis using custom-written scripts in Matlab and ImageJ plugin (FRET Analyzer). For RAP1/2 probes, images were sorted into donor, FRET, and acceptor channels. Masks were drawn on top of the membrane covering both sides of the peri membrane space. For each set of images, the values for different channels were registered and the final FRET index was obtained ${ }^{90}$ by correcting the bleed-through using the equation $\frac{R=\text { FRET }-(\alpha \times C y P e t)-(\beta \times Y \mathrm{Pet})}{C y \mathrm{Pet}}$

For the vinculin tension sensor, to obtain FA masks, CLAHE and Laplacian of Gaussian (LoG) were performed on the eYFP channel, followed by applying a size and intensity filter on the resulting particles. Since the vinculin tension biosensor is a single-chain construct, FRET Index was calculated by dividing the FRET channel (donor excitation with acceptor emission) by the CFP channel (donor excitation with donor emission).

Molecular dynamics (MD) models. The computational model consists of simulating the mechanical rigidity of a cell membrane based on using an AFM tip to measure force as a function of its deformation. MD models with the approximation of macromolecular systems $5^{52,53}$ were used to simulate the dynamics of a cell composed of membranes and actin filaments in two dimensions.

Cell model. We developed a membrane model that can reproduce the main features of the mechanical structure of a cell. The cell membrane, nuclear membrane, and actin filaments are modeled as being formed by beads connected by springs. Specifically, the cell membrane is composed of 201 beads connected by springs forming a diameter of $5.25 \mu \mathrm{m}$. To describe the nuclear membrane, it was necessary to use 33 beads connected by springs, generating a diameter of $1.5 \mu \mathrm{m}$ for the nucleus. Finally, the actin filaments, which are radial in the initial position, are separated into two types: type I filament has one end attached to the nuclear membrane and one end free, while type II filament has both ends free. For each type I configuration, we have two type II configurations interspersed, as depicted in Fig. 5c. The type I filament contains ten beads with a total length of $1.5 \mu \mathrm{m}$, while type II contains eight beads and a length of $1.2 \mu \mathrm{m}$.

To facilitate the simulation, each bead was labeled with one of three different numerical marks. All beads that make up the cell membrane are marked as 0 . The beads forming the actin filament and nuclear membrane were marked as 1 . The ends of actin filaments near the cell membrane were labeled as 3 . The elastic constants responsible for the bonds between each bead can be separated into three types: the elastic constant between the beads that make up the membranes (referred to as $\left.\kappa_{F 00}\right)$; the elastic constant that forms the actin filaments $\left(\kappa_{F 11}\right)$, except the head next to the cell membrane; and the elastic constant between the head of the filament and the nearest membrane bead $\left(\kappa_{F 13}\right)$. The relation between the constant modules in the simulation is given by: $\kappa_{F 11}=\kappa_{F 00}$ and $\kappa_{F 13}=5 \kappa_{F 11}$.

Potential model. To calculate the forces capable of creating the basic structure of a cell, it is necessary to define the potential of attraction and repulsion between the beads of type 0,1 , and 3 (see above).

We based our system on a Bead-Spring model of polymers, also known as the coarse-grained model. In this model, we have the following potentials based on corresponding equations:

Lennard-Jones (L-J) potential (1), where the parameter $r$ is the distance between particles (beads), while $\sigma$ is the equilibrium distance, and $\varepsilon$ the well depth energy.

$$
\mathrm{U}_{\mathrm{LJ}}(r)=\varepsilon\left[\left(\frac{\sigma}{r}\right)^{12}-2\left(\frac{\sigma}{r}\right)^{6}\right]
$$

Weeks-Chandler-Anderson (WCA) (2), representing the repulsive part of L-J potential:

$$
\mathrm{U}_{\mathrm{WCA}}(r)=\left\{\begin{array}{c}
\mathrm{U}_{\mathrm{LJ}}(r)+\varepsilon, \text { if } r<\sigma \\
0, \text { if } r \geq \sigma
\end{array}\right\}
$$

Finite extensible nonlinear elastic (FENE) potential (3), where $\kappa_{\mathrm{F}}$ is the FENE spring constant, $\Delta r^{\max }$ is a maximal extension/compression, and $r_{0}$ is equilibrium position.

$$
\mathrm{U}_{\mathrm{FENE}}(r)=\frac{-\kappa_{F}\left(\Delta r_{\max }\right)^{2}}{2} \log \left[1-\left(\frac{r-r_{0}}{\Delta r_{\max }}\right)^{2}\right]
$$

Bend potential (4), related to torsional potential. It represents the energy associated with bending between three neighboring beads and $\kappa$ is the bend spring constant, where $\theta$ is the angle between three consecutive neighbor beads and $\theta_{0}$ is the equilibrium angle.

$$
\mathrm{U}_{\mathrm{BEND}}(\theta)=\frac{\kappa_{B}}{2}\left(\cos (\theta)-\cos \left(\theta_{0}\right)\right)^{2}
$$

The potentials are shown as a function of the distances between beads for various values of $\kappa_{F i j}, \kappa_{B i j}, \varepsilon_{i j}, \sigma_{i j}, \Delta r \max _{i j}$, with $i, j=0,1,3$ (types of beads, see above) (Fig. S14). All quantities are dimensionless and are represented by the asterisk symbol.

AFM tip model. To model the tip of an AFM, we used two elastic constants, one in $\mathrm{x}$ direction $\left(k_{\mathrm{x}}\right)$ and one in $y$ direction $\left(k_{\mathrm{y}}\right)$, noting that we are working on a 2-D problem. The spring are clamped into an apparatus and the assembly moves in the direction of the cell membrane with constant velocity $\mathrm{V}$. The force on the membrane is measured as a function of the deformations of the springs. The tip of the AFM is shaped like a bead and marked as 7 .

Numerical simulation. With the definitions given above and appropriately choosing the parameters for $\sigma$, it is possible to analyze the stiffness behavior of the cell using the AFM tip. The proposed model allows us to attract energy between the membranes $\left(\varepsilon_{00}\right)$ and also the attraction energy that the actin filament head makes in the membrane $\left(\varepsilon_{03}\right)$. The results of the force made by the AFM tip on the membrane as a function of its distance traveled, for both cases, are shown in graphs in figures. To facilitate analysis both graphs were plotted on the same scale.

Statistics and reproducibility. Data were presented as mean \pm SEM unless indicated otherwise. One-way analysis of variance (ANOVA) with Tukey's or Dunnett's post hoc test or Kruskal-Wallis test with Dunn's multiple comparisons test were performed for data with more than two groups. Unpaired $t$-test (two-sided) or Mann-Whitney test was applied when comparing data from two groups. For studies with repeated measures (RM), a two-way ANOVA RM and Bonferroni post hoc test was performed. Statistical analyses were performed using GraphPad Prism 8 statistical software. Statistical significance was considered as $P<0.05\left(^{*}\right) ; P<0.01$ $(* *) ; P<0.001(* * *)$. Sample sizes and statistical details are indicated in figure legends.

All cell culture experiments presented in the manuscript were repeated at least three times independently with similar results.

Reporting Summary. Further information on research design is available in the Nature Research Reporting Summary linked to this article.

\section{Data availability}

The RNA-seq data generated in this study have been deposited in the NCBI Gene Expression Omnibus (GEO) database under accession code "GSE158017". The hg38 human reference genome was obtained from Ensembl Release 90 (https:// useast.ensembl.org/index.html).

Source data underlying graphs and full WB images are provided in Source Data. All other data that support the findings of this study are available from the corresponding author R.H.F. upon reasonable request. Source data are provided with this paper.

\section{Code availability}

Molecular dynamics (MD) codes have been deposited at Github (github.com/diasrodri/ SimCellMD-1) and Zenodo (zenodo.org/record/4977917\#.YMyfWTZKjyU) ${ }^{91}$.

Received: 13 February 2021; Accepted: 29 September 2021; Published online: 14 October 2021

\section{References}

1. Mammoto, T. \& Ingber, D. E. Mechanical control of tissue and organ development. Development 137, 1407-1420 (2010).

2. DuFort, C. C., Paszek, M. J. \& Weaver, V. M. Balancing forces: architectural control of mechanotransduction. Nat. Rev. Mol. Cell Biol. 12, 308-319 (2011).

3. Hannezo, E. \& Heisenberg, C. P. Mechanochemical feedback loops in development and disease. Cell 178, 12-25 (2019).

4. Pilot, F. \& Lecuit, T. Compartmentalized morphogenesis in epithelia: from cell to tissue shape. Dev. Dyn. 232, 685-694 (2005).

5. Fernandez-Gonzalez, R., Simoes, Se. M., Röper, J. C., Eaton, S. \& Zallen, J. A. Myosin II dynamics are regulated by tension in intercalating cells. Dev. Cell 17, 736-743 (2009)

6. Rosowski, K. A., Mertz, A. F., Norcross, S., Dufresne, E. R. \& Horsley, V. Edges of human embryonic stem cell colonies display distinct mechanical properties and differentiation potential. Sci. Rep. 5, 14218 (2015).

7. Meng, Z. et al. RAP2 mediates mechanoresponses of the Hippo pathway. Nature 560, 655-660 (2018).

8. $\mathrm{Li}, \mathrm{D}$. et al. Integrated biochemical and mechanical signals regulate multifaceted human embryonic stem cell functions. J. Cell Biol. 191, 631-644 (2010).

9. Krtolica, A. et al. Disruption of apical-basal polarity of human embryonic stem cells enhances hematoendothelial differentiation. Stem Cells 25, 2215-2223 (2007).

10. Nikolopoulou, E., Galea, G. L., Rolo, A., Greene, N. D. \& Copp, A. J. Neural tube closure: cellular, molecular and biomechanical mechanisms. Development 144, 552-566 (2017).

11. Daviaud, N., Chen, K., Huang, Y., Friedel, R. H. \& Zou, H. Impaired cortical neurogenesis in plexin-B1 and -B2 double deletion mutant. Dev. Neurobiol. 76, 882-899 (2016). 
12. Friedel, R. H. et al. Plexin-B2 controls the development of cerebellar granule cells. J. Neurosci. 27, 3921-3932 (2007).

13. Deng, S. et al. Plexin-B2, but not Plexin-B1, critically modulates neuronal migration and patterning of the developing nervous system in vivo. $J$. Neurosci. 27, 6333-6347 (2007).

14. Wansleeben, C. et al. An ENU-mutagenesis screen in the mouse: identification of novel developmental gene functions. PLoS ONE 6, e19357 (2011).

15. Hirschberg, A. et al. Gene deletion mutants reveal a role for semaphorin receptors of the plexin-B family in mechanisms underlying corticogenesis. Mol. Cell Biol. 30, 764-780 (2010).

16. Saha, B., Ypsilanti, A. R., Boutin, C., Cremer, H. \& Chedotal, A. Plexin-B2 regulates the proliferation and migration of neuroblasts in the postnatal and adult subventricular zone. J. Neurosci. 32, 16892-16905 (2012). 32/47/ 16892 [pii].

17. Huang, Y. et al. Plexin-B2 facilitates glioblastoma infiltration by modulating cell biomechanics. Commun. Biol. 4, 145 (2021).

18. Jiang, C. et al. Mechanochemical control of epidermal stem cell divisions by B-plexins. Nat. Commun. 12, 1308 (2021).

19. Gurrapu, S. \& Tamagnone, L. Transmembrane semaphorins: multimodal signaling cues in development and cancer. Cell Adh. Migr. 10, 675-691 (2016).

20. Koropouli, E. \& Kolodkin, A. L. Semaphorins and the dynamic regulation of synapse assembly, refinement, and function. Curr. Opin. Neurobiol. 27, 1-7 (2014).

21. Kumanogoh, A. Semaphorins - A Diversity of Emerging Physiological and Pathological Activities (Springer, 2015).

22. Kumanogoh, A. \& Kikutani, H. Immunological functions of the neuropilin and plexins as receptors for semaphorins. Nat. Rev. Immunol. 13, 802-814 (2013).

23. Negishi-Koga, T. \& Takayanagi, H. Bone cell communication factors and Semaphorins. Bonekey Rep. 1, 183 (2012)

24. Pasterkamp, R. J. Getting neural circuits into shape with semaphorins. Nat. Rev. Neurosci. 13, 605-618 (2012).

25. Sakurai, A., Doçi, C. L., Doci, C. \& Gutkind, J. S. Semaphorin signaling in angiogenesis, lymphangiogenesis and cancer. Cell Res. 22, 23-32 (2012).

26. Tran, T. S., Kolodkin, A. L. \& Bharadwaj, R. Semaphorin regulation of cellular morphology. Annu. Rev. Cell Dev. Biol. 23, 263-292 (2007).

27. Worzfeld, T. \& Offermanns, S. Semaphorins and plexins as therapeutic targets. Nat. Rev. Drug Disco. 13, 603-621 (2014).

28. Xia, J. et al. Semaphorin-Plexin signaling controls mitotic spindle orientation during epithelial morphogenesis and repair. Dev. Cell 33, 299-313 (2015).

29. Junqueira Alves, C., Yotoko, K., Zou, H. \& Friedel, R. H. Origin and evolution of plexins, semaphorins, and Met receptor tyrosine kinases. Sci. Rep. 9, 1970 (2019).

30. Junqueira Alves, C. et al. Evolution and diversity of semaphorins and plexins in choanoflagellates. Genome Biol. Evol. https://doi.org/10.1093/gbe/evab035 (2021).

31. Kong, Y. et al. Structural basis for plexin activation and regulation. Neuron 91 , 548-560 (2016).

32. Suzuki, K. et al. Structure of the plexin ectodomain bound by semaphorinmimicking antibodies. PLoS ONE 11, e0156719 (2016).

33. Pascoe, H. G., Wang, Y. \& Zhang, X. Structural mechanisms of plexin signaling. Prog. Biophys. Mol. Biol. 118, 161-168 (2015).

34. Kooistra, M. R., Dubé, N. \& Bos, J. L. Rap1: a key regulator in cell-cell junction formation. J. Cell Sci. 120, 17-22 (2007).

35. Bos, J. L. From Ras to Rap and back, a journey of 35 Years. Cold Spring Harb. Perspect Med. https://doi.org/10.1101/cshperspect.a031468 (2018).

36. Sun, Y. et al. Hippo/YAP-mediated rigidity-dependent motor neuron differentiation of human pluripotent stem cells. Nat. Mater. 13, 599-604 (2014).

37. Dupont, S. et al. Role of YAP/TAZ in mechanotransduction. Nature 474, 179-183 (2011).

38. Mascetti, V. L. \& Pedersen, R. A. Naiveté of the human pluripotent stem cell. Nat. Biotechnol. 32, 68-70 (2014).

39. Franco, M. \& Tamagnone, L. Tyrosine phosphorylation in semaphorin signalling: shifting into overdrive. EMBO Rep. 9, 865-871 (2008). embor2008139[pii].

40. Sever, M., Gunay, G., Guler, M. O. \& Tekinay, A. B. Tenascin-C derived signaling induces neuronal differentiation in a three-dimensional peptide nanofiber gel. Biomater. Sci. 6, 1859-1868 (2018).

41. Totsukawa, G. et al. Distinct roles of ROCK (Rho-kinase) and MLCK in spatial regulation of MLC phosphorylation for assembly of stress fibers and focal adhesions in 3T3 fibroblasts. J. Cell Biol. 150, 797-806 (2000).

42. Lukinavičius, G. et al. Fluorogenic probes for live-cell imaging of the cytoskeleton. Nat. Methods 11, 731-733 (2014).

43. Azeloglu, E. U. \& Costa, K. D. Atomic force microscopy in mechanobiology: measuring microelastic heterogeneity of living cells. Methods Mol. Biol. 736, 303-329 (2011).
44. Riedl, J. et al. Lifeact: a versatile marker to visualize F-actin. Nat. Methods 5, 605-607 (2008)

45. Grashoff, C. et al. Measuring mechanical tension across vinculin reveals regulation of focal adhesion dynamics. Nature 466, 263-266 (2010).

46. Cowin, P. \& Burke, B. Cytoskeleton-membrane interactions. Curr. Opin. Cell Biol. 8, 56-65 (1996).

47. Porazinski, S. et al. YAP is essential for tissue tension to ensure vertebrate 3D body shape. Nature 521, 217-221 (2015).

48. Singh, P., Carraher, C. \& Schwarzbauer, J. E. Assembly of fibronectin extracellular matrix. Annu. Rev. Cell Dev. Biol. 26, 397-419 (2010).

49. Rolo, A., Skoglund, P. \& Keller, R. Morphogenetic movements driving neural tube closure in Xenopus require myosin IIB. Dev. Biol. 327, 327-338 (2009).

50. Foty, R. A simple hanging drop cell culture protocol for generation of 3D spheroids. J. Vis. Exp. https://doi.org/10.3791/2720 (2011).

51. Meltzer, S. et al. Epidermis-derived semaphorin promotes dendrite selfavoidance by regulating dendrite-substrate adhesion in Drosophila sensory neurons. Neuron 89, 741-755 (2016).

52. Bachmann, M. Thermodynamics and Statistical Mechanics of Macromolecular Systems (Cambridge Univ. Press, 2014).

53. Binder, K. Monte Carlo and Molecular Dynamics Simulations in Polymer Science (Oxford Univ. Press, 1995).

54. Lecuit, T. \& Lenne, P. F. Cell surface mechanics and the control of cell shape, tissue patterns and morphogenesis. Nat. Rev. Mol. Cell Biol. 8, 633-644 (2007).

55. Shang, X. et al. Small-molecule inhibitors targeting G-protein-coupled Rho guanine nucleotide exchange factors. Proc. Natl Acad. Sci. USA 110, 3155-3160 (2013).

56. Zhou, Y., Gunput, R. A. \& Pasterkamp, R. J. Semaphorin signaling: progress made and promises ahead. Trends Biochem Sci. 33, 161-170 (2008).

57. Oinuma, I., Ishikawa, Y., Katoh, H. \& Negishi, M. The semaphorin 4D receptor plexin-B1 is a GTPase activating protein for R-Ras. Science 305, 862-865 (2004)

58. Saito, Y., Oinuma, I., Fujimoto, S. \& Negishi, M. Plexin-B1 is a GTPase activating protein for $\mathrm{M}$-Ras, remodelling dendrite morphology. EMBO Rep. 10, 614-621 (2009)

59. Wang, Y. et al. Plexins are GTPase-activating proteins for Rap and are activated by induced dimerization. Sci. Signal 5, ra6 (2012). 5/207/ra6[pii].

60. Vikis, H. G., Li, W. \& Guan, K. L. The plexin-B1/Rac interaction inhibits PAK activation and enhances Sema4D ligand binding. Genes Dev. 16, 836-845 (2002).

61. Swiercz, J. M., Kuner, R. \& Offermanns, S. Plexin-B1/RhoGEF-mediated RhoA activation involves the receptor tyrosine kinase ErbB-2. J. Cell Biol. 165, 869-880 (2004).

62. Sudol, M. et al. Characterization of the mammalian YAP (Yes-associated protein) gene and its role in defining a novel protein module, the WW domain. J. Biol. Chem. 270, 14733-14741 (1995).

63. Zhao, B., Lei, Q. Y. \& Guan, K. L. The Hippo-YAP pathway: new connections between regulation of organ size and cancer. Curr. Opin. Cell Biol. 20, 638-646 (2008).

64. Pagliari, S. et al. YAP-TEAD1 control of cytoskeleton dynamics and intracellular tension guides human pluripotent stem cell mesoderm specification. Cell Death Differ. 28, 1193-1207 (2021).

65. Chaqour, B. \& Goppelt-Struebe, M. Mechanical regulation of the Cyr61/ CCN1 and CTGF/CCN2 proteins. FEBS J. 273, 3639-3649 (2006).

66. Lancaster, M. A. et al. Cerebral organoids model human brain development and microcephaly. Nature 501, 373-379 (2013).

67. Lancaster, M. \& Knoblich, J. Generation of cerebral organoids from human pluripotent stem cells. Nat. Protoc. 9, 2329-2340 (2014).

68. Sasai, Y. Cytosystems dynamics in self-organization of tissue architecture. Nature 493, 318-326 (2013)

69. Parsons, J. T., Horwitz, A. R. \& Schwartz, M. A. Cell adhesion: integrating cytoskeletal dynamics and cellular tension. Nat. Rev. Mol. Cell Biol. 11, 633-643 (2010).

70. Daley, W. P., Peters, S. B. \& Larsen, M. Extracellular matrix dynamics in development and regenerative medicine. J. Cell Sci. 121, 255-264 (2008).

71. Tamagnone, L. et al. Plexins are a large family of receptors for transmembrane, secreted, and GPI-anchored semaphorins in vertebrates. Cell 99, 71-80 (1999).

72. Mehta, V. et al. The guidance receptor plexin D1 is a mechanosensor in endothelial cells. Nature 578, 290-295 (2020).

73. Fletcher, D. A. \& Mullins, R. D. Cell mechanics and the cytoskeleton. Nature 463, 485-492 (2010).

74. Keung, A. J., Asuri, P., Kumar, S. \& Schaffer, D. V. Soft microenvironments promote the early neurogenic differentiation but not self-renewal of human pluripotent stem cells. Integr. Biol. 4, 1049-1058 (2012).

75. Halder, G., Dupont, S. \& Piccolo, S. Transduction of mechanical and cytoskeletal cues by YAP and TAZ. Nat. Rev. Mol. Cell Biol. 13, 591-600 (2012). 
76. Mitz, A. R. et al. Identification of $22 \mathrm{q} 13$ genes most likely to contribute to Phelan McDermid syndrome. Eur. J. Hum. Genet. 26, 293-302 (2018).

77. Gurrapu, S., Pupo, E., Franzolin, G., Lanzetti, L. \& Tamagnone, L. Sema4C/ PlexinB2 signaling controls breast cancer cell growth, hormonal dependence and tumorigenic potential. Cell Death Differ. 25, 1259-1275 (2018).

78. Le, A. P. et al. Plexin-B2 promotes invasive growth of malignant glioma. Oncotarget 6, 7293-7304 (2015).

79. Thomson, J. A. et al. Embryonic stem cell lines derived from human blastocysts. Science 282, 1145-1147 (1998).

80. Ran, F. A. et al. Genome engineering using the CRISPR-Cas9 system. Nat. Protoc. 8, 2281-2308 (2013).

81. Sanjana, N. E., Shalem, O. \& Zhang, F. Improved vectors and genome-wide libraries for CRISPR screening. Nat. Methods 11, 783-784 (2014).

82. Zhao, B. et al. TEAD mediates YAP-dependent gene induction and growth control. Genes Dev. 22, 1962-1971 (2008).

83. Ge, X. et al. LIM-nebulette reinforces podocyte structural integrity by linking actin and vimentin filaments. J. Am. Soc. Nephrol. 31, 2372-2391 (2020).

84. Stringer, C., Wang, T., Michaelos, M. \& Pachitariu, M. Cellpose: a generalist algorithm for cellular segmentation. Nat. Methods 18, 100-106 (2021).

85. Kim, D., Paggi, J. M., Park, C., Bennett, C. \& Salzberg, S. L. Graph-based genome alignment and genotyping with HISAT2 and HISAT-genotype. Nat. Biotechnol. 37, 907-915 (2019).

86. Liao, Y., Smyth, G. K. \& Shi, W. The R package Rsubread is easier, faster, cheaper and better for alignment and quantification of RNA sequencing reads. Nucleic Acids Res. 47, e47 (2019).

87. Love, M. I., Huber, W. \& Anders, S. Moderated estimation of fold change and dispersion for RNA-seq data with DESeq2. Genome Biol. 15, 550 (2014).

88. Xia, J., Gill, E. E. \& Hancock, R. E. NetworkAnalyst for statistical, visual and network-based meta-analysis of gene expression data. Nat. Protoc. 10, 823-844 (2015).

89. O'Shaughnessy, E. C. et al. Software for lattice light-sheet imaging of FRET biosensors, illustrated with a new Rap1 biosensor. J. Cell Biol. 218, 3153-3160 (2019).

90. Hodgson, L., Shen, F. \& Hahn, K. Biosensors for characterizing the dynamics of rho family GTPases in living cells. Curr. Protoc. Cell Biol. https://doi.org/ 10.1002/0471143030.cb1411s46 (2010).

91. Alves Dias, R. \& Rodrigues Furtado de Mendonça, J. P. diasrodri/SimCellMD1: first release. Zenodo https://doi.org/10.5281/zenodo.4977917 (2021).

\section{Acknowledgements}

We thank E. O'Shaughnessy and K. Hahn, the University of North Carolina at Chapel Hill, for Rap FRET plasmids. The work was supported by NINDS awards to H.Z. (NS107462) and R.H.F (NS092735), and NY state DOH award to H.Z. (C32242GG); NIDDK R01s DK106035 to G.L.G and DK118222 to E.U.A.; NIH/NIDCR T32HD075735 to R.J.W.; CAPES, FAPEMIG, and CNPq funding to R.A.D. and J.P.R.F.M. Additional fellowship support was provided for C.J.A. by the National Council for Scientific and Technological Development (CNPq, Brazil; \#200358/2015-4) and for
R.D. by the São Paulo Research Foundation (FAPESP, Brazil; 2016/07541-5). S. Kesari was supported by funds from the Saint John's Health Center Foundation.

\section{Author contributions}

C.J.A., R.H.F., and H.Z. designed the overall study, analyzed and compiled data, and wrote the manuscript. C.J.A., R.D., S. Kang, and T.H. conducted experiments and performed data analyses. R.D and E.A.S. designed calcium experiments. J.H. and S.D. performed TIRF experiments. R.J.W. performed AFM measurements and surface topology study. R.T. performed cell cytometry. J.H., R.J.W., G.L.G., and E.U.A. designed executed, and analyzed AFM, FRET, and TIRF mechanobiological studies. R.A.D. and J.P.M. performed the mathematical modeling. A.W. and S.K. performed RNA-sequencing, and A.R. and L.S. conducted bioinformatics analyses. All authors discussed manuscript preparation.

\section{Competing interests}

The authors declare no competing interests.

\section{Additional information}

Supplementary information The online version contains supplementary material available at https://doi.org/10.1038/s41467-021-26296-7.

Correspondence and requests for materials should be addressed to Hongyan Zou or Roland H. Friedel.

Peer review information Nature Communications thanks Yoichi Kosodo and the other anonymous reviewer(s) for their contribution to the peer review this work.

Reprints and permission information is available at http://www.nature.com/reprints

Publisher's note Springer Nature remains neutral with regard to jurisdictional claims in published maps and institutional affiliations.

(c) (i) Open Access This article is licensed under a Creative Commons cc Attribution 4.0 International License, which permits use, sharing, adaptation, distribution and reproduction in any medium or format, as long as you give appropriate credit to the original author(s) and the source, provide a link to the Creative Commons license, and indicate if changes were made. The images or other third party material in this article are included in the article's Creative Commons license, unless indicated otherwise in a credit line to the material. If material is not included in the article's Creative Commons license and your intended use is not permitted by statutory regulation or exceeds the permitted use, you will need to obtain permission directly from the copyright holder. To view a copy of this license, visit http://creativecommons.org/ licenses/by/4.0/.

(C) The Author(s) 2021 